Murilo Pereira Soares

\title{
On the Solution Variability Reduction of Stochastic dual Dynamic Programming Applied to Energy Planning
}

\author{
Tese de Doutorado
}

Thesis presented to the Programa de Pós-Graduação em Engenharia Elétrica of the Departamento de Engenharia Elétrica do Centro Técnico Científico da PUC-Rio, as partial fulfillment of the requirements for the degree of Doutor em Engenharia Elétrica

Advisor : Prof. Alexandre Street de Aguiar Co-Advisor: $\quad$ Prof. Davi Michel Valladão 


\title{
Murilo Pereira Soares
}

\section{On the Solution Variability Reduction of Stochastic dual Dynamic Programming Applied to Energy Planning}

\section{TESE DE DOUTORADO}

Thesis presented to the Programa de Pós-Graduação em Engenharia Elétrica of the Departamento de Engenharia Elétrica do Centro Técnico Científico da PUC-Rio, as partial fulfillment of the requirements for the degree of Doutor

\author{
Prof. Alexandre Street de Aguiar \\ Advisor \\ Departamento de Engenharia Elétrica - PUC-Rio \\ Prof. Davi Michel Valladão \\ Co-Advisor \\ Departamento de Engenharia Industrial \\ Dr. Joari Paulo da Costa \\ Operador Nacional do Sistema Elétrico - Matriz
}

Dr. Vitor Luiz de Matos

PLAN4 Engenharia

Dr. André Luiz Diniz Souto Lima

CEPEL

Prof. Delberis Araújo Lima

Departamento de Engenharia Elétrica - PUC-Rio

Prof. José Eugênio Leal

Coordinator of the Centro Técnico Científico da PUC-Rio 
All rights reserved.

\section{Murilo Pereira Soares}

Murilo Soares graduated from the Federal University of Juiz de Fora (UFJF) in Electrical Engineering. In 2008 he obtained a Master degree the PUC-Rio in Electrical Engineering. From 2005 to 2013 he worked at the Brazilian electrical system independent operator (ONS - Operador Nacional do Sistema Elétrico) and later in 2013 he joined the market operations team of Statkraft.

Ficha Catalográfica

Pereira Soares, Murilo

On the Solution Variability Reduction of Stochastic dual Dynamic Programming Applied to Energy Planning / Murilo Pereira Soares ; advisor: Prof. Alexandre Street de Aguiar; co-advisor: Prof. Davi Michel Valladão. - 2015.

118 f. : il. (color); $30 \mathrm{~cm}$

Tese (doutorado)-Pontifícia Universidade Católica do Rio de Janeiro, Rio de Janeiro, 2015.

Inclui bibliografia

1. Engenharia Elétrica - Teses. 2. Programação estocástica. 3. Programação Dinâmica Dual Estocástica. 4. Aversão a Risco. 5. Pesquisa Operacional em Energia. 6. Planejamento da Operação Hidrotérmica. I. de Aguiar, Alexandre Street. II. Valladão, Davi Michel. III. Pontifícia Universidade Católica do Rio de Janeiro. Departamento de Engenharia Elétrica. IV. Título. 


\section{Acknowledgments}

Foremost, I would like to express my sincere gratitude to my advisor Alexandre Street and co-advisor Davi Valladão for the continuous support of my doctoral study and research, and for the patience with a student that, due to work duties, was almost always absent.

My sincere thanks also goes to Joari, who was a great advisor during almost my entire career, and with whom I learned a lot.

I thank my Brunos friends for their support since the beginning of my doctoral studies. From them I heard things like "Are you crazy? Are you sure you want to do it? ... Ok, you're right, you always wanted it." and "Do it now, let's work together in the future!". They'll know who said what...

During most part of this work I was working at ONS, and I'm also very thankful for the time I spent there, for the support and for bringing up the main theme of this thesis. I also thank all my colleagues there, particularly from GMC2 area, Alberto, Alessandra, Cadu, Cândida, Carlos, Cecília, Francislene, Maria Helena, Mônica and Sérgio. This work wouldn't exist without all the discussions and time we've worked together.

Another person I must thank is Prof. Alex Shapiro, from Georgia Tech. His patient way of explaining difficult things allowed me to learn a lot during the time we worked together.

During the latest quarter of this work I joined SN Power, now Statkraft, and I was also encouraged and supported to do my best in this work, even though this thesis was somehow competing with my need to learn about a new world in my career. I thank Lukas, Bruno, Leonardo, Ingrid, Paula, Pedro, Rodrigo and Rogério for making this transition easier for me.

I cannot forget the helpful discussions with Bernardo, one of which, among drinks and food in the IEEE PES General Meeting, ended up in the suggestion of a volatility measure used in this work. I must also thank Sérgio Granville, whose question during my thesis proposal actually turned out to be a suggestion that ended up in the first presented proposal.

I would like to thank my family: my parents Fatinha and Plínio, for giving birth to me in the first place and for educating me to be the person I am today; and my brothers Gabriel and Fabrício for being my friends even now that we are more distant and regardless of my reluctance of using the phone.

Last but not the least, I must thank my wife, Victória, who has patiently followed my struggle to find strength to work on this thesis after many workdays and during weekends. I'll never forget her effort for this thesis to be completed, and I promise I'll take her to the beach whenever she wants! 


\section{Abstract}

Pereira Soares, Murilo; de Aguiar, Alexandre Street; Valladão, Davi Michel. On the Solution Variability Reduction of Stochastic dual Dynamic Programming Applied to Energy Planning. Rio de Janeiro, 2015. 118p. Tese de Doutorado - Departamento de Engenharia Elétrica, Pontifícia Universidade Católica do Rio de Janeiro.

In the hydrothermal energy operation planning of Brazil and other hydro-dependent countries, Stochastic Dual Dynamic Programming (SDDP) computes a risk-averse optimal policy that often considers river-inflow autoregressive models. In practical applications, these models induce an undesirable variability of primal (thermal generation) and dual (marginal cost and spot price) solutions, which are highly sensitive to changes in current inflow conditions. In this work, we propose two differing approaches to stabilize SDDP solutions to the energy operation planning problem: the first approach aims at regularizing primal variables by considering an additional penalty on thermal dispatch revisions over time. The second approach indirectly reduces thermal generation and marginal cost variability by disregarding past inflow information in the cost-to-go function and compensating it with an increase in risk aversion. For comparison purposes, we assess solution quality with a set of proposed indexes summarizing each important aspect of a hydrothermal operation planning policy. In conclusion, we show it is possible to obtain highquality solutions in comparison to current benchmarks and with significantly reduced variability.

\section{Keywords}

Stochastic programming; Stochastic Dual Dynamic Programming; Risk aversion; Operational Research in Energy; Hydrothermal Energy Operation Planning; 


\section{Resumo}

Pereira Soares, Murilo; de Aguiar, Alexandre Street; Valladão, Davi Michel. Redução da Variabilidade da Solução da Programação Dinâmica Dual Estocástica Aplicada ao Planejamento da Operação de Sistemas Hidrotérmicos. Rio de Janeiro, 2015. 118p. Tese de Doutorado - Departamento de Engenharia Elétrica, Pontifícia Universidade Católica do Rio de Janeiro.

No planejamento da operação hidrotérmica brasileiro, assim como em outros países hidro dependentes, a Programação Dinâmica Dual Estocástica (PDDE) é utilizada para calcular uma política ótima avessa a risco que, muitas vezes, considera modelos autorregressivos para modelagem das afluências às hidrelétricas. Em aplicações práticas, estes modelos podem induzir a uma variabilidade indesejável de variáveis primais (geração térmica) e duais (custo marginal e preço spot), que são altamente sensíveis a mudanças nas condições iniciais das vazões. Neste trabalho, são propostas duas abordagens diferentes para estabilizar as soluções da PDDE no problema de planejamento da operação energética: a primeira abordagem visa regularizar variáveis primais considerando uma penalidade adicional sobre as mudanças no despacho térmico ao longo do tempo. A segunda abordagem reduz indiretamente a variabilidade da geração térmica e do custo marginal ao ignorar informações de afluências passadas na função de custo futuro e compensando-a com um aumento na aversão ao risco. Para fins de comparação, a qualidade solução foi avaliada com um conjunto de índices propostos que resumem cada aspecto importante de uma política de planejamento hidrotérmico. Em conclusão, mostramos que é possível obter soluções com boa qualidade em comparação com benchmarks atuais e com uma redução significativa variabilidade.

\section{Palavras-chave}

Programação estocástica; Programação Dinâmica Dual Estocástica; Aversão a Risco; Pesquisa Operacional em Energia; Planejamento da Operação Hidrotérmica; 


\section{Contents}

1 Introduction $\quad 12$

1.1 Motivation 12

$\begin{array}{lll}1.2 & \text { Objective } & 13\end{array}$

1.3 Contributions and Work Organization 14

2 Notation 16

3 Overview of the Brazilian Hydrothermal Operation Planning $\quad 18$

$\begin{array}{lll}3.1 & \text { Physical characteristics } & 18\end{array}$

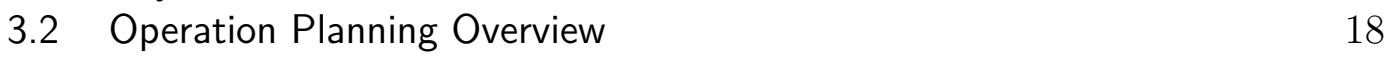

$\begin{array}{ll}3.3 \text { Solution variability } & 27\end{array}$

4 SDDP algorithm and time dependency $\quad 38$

$\begin{array}{lll}4.1 & \text { Independent SDDP algorithm } & 39\end{array}$

4.2 SDDP algorithm with time dependent time series model 45

@ $\quad 5$ On the solution variability reduction $\quad 52$

5.1 Thermal generation stabilization: risk-averse SDDP with increased state space 52

5.2 Thermal generation and marginal costs stabilization: risk-averse backward-independent SDDP algorithm 55

6 Solution Quality Indexes for Hydrothermal Operation Planning Problem 62

6.1 Solution predictability and variability 63

6.2 Cost-based indexes 69

6.3 Continuity of energy supply 71

$\begin{array}{lll}6.4 & \text { Summary indexes } & 73\end{array}$

$7 \quad$ Computational experiments $\quad 75$

$\begin{array}{lll}7.1 & \text { Case studies description } & 75\end{array}$

$\begin{array}{lll}7.2 & \text { Comparison between chosen case studies } & 79\end{array}$

8 Conclusions and Future Work $\quad 97$

$\begin{array}{lll}8.1 \text { Conclusions } & 97\end{array}$

$\begin{array}{lll}8.2 & \text { Future Work } & 98\end{array}$

$\begin{array}{ll}\text { A Publications } & 100\end{array}$

B Autoregressive model impact on a general problem 102

$\begin{array}{ll}\text { C Case study parametrization } & 104\end{array}$

C.1 Thermal generation stabilization 104

$\begin{array}{ll}\text { C.2 Risk averse backward independent SDDP } & 109\end{array}$ 


\section{List of Figures}

3.1 Brazilian interconnected power system 19

3.2 Brazilian interconnected power system representation 20

3.3 Thermal generation availability and unit variation cost - May/2015 32

3.4 Energy inflows in Southeast system as a \% of the monthly historical average 34

3.5 Weekly thermal generation and variation - Oct/2012-Mar/2013 35

3.6 Weekly marginal costs and variation - Oct/2012-Mar/2013 37

6.1 1-SAFE index estimation - dashed red line is the regression including the red triangle spilling points; the blue line is the adopted regression, discarding such points

6.2 Hypothetical energy stored levels average, 5\% and 95\% quantiles for each stage [MWmonth]

6.3 Second stage solution PLD

6.4 Variable results for a given scenario 67

6.5 Variability index 68

6.6 Average and 99\% quantile costs for each stage 69

6.7 Policy values $\left[\mathrm{R} \$ \times 10^{6}\right]$ and its $95 \%$ confidence interval $\quad 70$

6.8 Stored Energy Volume Analysis $\quad 72$

$\begin{array}{ll}6.9 \text { Annual risks of deficit [\%] } & 74\end{array}$

7.1 Lower bound over the iteration process 79

7.2 Simulation cost over the iteration process (cost for 1 forward scenario) 79

7.3 Thermal Generation volatility 1-SAFE measure over time 81

7.4 PLD volatility 1-SAFE measure over time 82

7.5 Thermal Generation average and $5 \%$ and $95 \%$ quantiles 82

7.6 PLDs average and 5\% and 95\% quantiles 83

$\begin{array}{lll}7.7 & \text { Thermal generation variability } & 84\end{array}$

7.8 Thermal generation for simulation with historical inflows occurred between 2001-2005 85

7.9 PLD variability 85

7.10 PLDs for simulation with historical inflows occurred between 20012005

$\begin{array}{ll}7.11 \text { Second stage thermal generation [MWmonth] } & 87\end{array}$

7.12 Second stage PLDs $\quad 88$

7.13 Average and 99\% quantile costs for each stage $\quad 89$

7.14 Average, 5\% and 95\% quantile stored volumes for each stage $\begin{array}{ll}\text { [MWmonth] } & 91\end{array}$

7.15 Stored Energy Quality Index for each stage $\quad 92$

7.16 Annual risks of deficit [\%] 93

7.17 Total stored energy for simulation with historical inflows occurred between 1979-1983

7.18 Total spilled Energy for simulation with historical inflows occurred between 1979-1983 
7.19 Total stored energy for simulation with historical inflows occurred between 2004-2008

7.20 Total Brazilian stored energy for simulation with historical inflows occurred between 2001-2005

7.21 Total Brazilian stored energy for simulation with historical inflows occurred between 1952-1956

7.22 Total Brazilian energy deficit for simulation with historical inflows occurred between 1952-1956

C.1 Thermal generation approach - Policy values and 95\% confidence interval

C.2 Thermal generation approach - Annual risks of deficit

C.3 Thermal generation approach - Thermal generation 1-SAFE index

108

C.4 Thermal generation approach - PLD 1-SAFE index 109

C.5 BW-I approach - Policy values and 95\% confidence interval 110

C.6 BW-I approach - Annual risks of deficit

C.7 BW-I approach - Thermal generation 1-SAFE index

C.8 BW-I approach - PLD 1-SAFE index 


\section{List of Tables}

$\begin{array}{lll}7.1 & \text { Equivalent reservoirs data [MWmonth] } & 75\end{array}$

$\begin{array}{lll}7.2 & \text { Thermal plants summary } & 76\end{array}$

$\begin{array}{lll}7.3 & \text { Deficit costs and depths } & 76\end{array}$

7.4 Interconnection limits between systems [MWavg] 76

$\begin{array}{ll}7.5 \text { Summary results indexes } & 80\end{array}$

7.6 Uncertainty indexes 83

7.7 Thermal Generation Average Variability 84

7.8 Thermal Generation Variability Standard Deviation 84

7.9 PLD Average Variability 86

7.10 PLD Variability Standard Deviation 86

7.11 Second stage thermal generation statistics [R\$/MWh] 87

$\begin{array}{ll}7.12 \text { Second stage PLDs statistics } & 88\end{array}$

7.13 Policy values $\left[R \$ \times 10^{6}\right] \quad 90$

$7.145 \%$ Conditional Value at Risk $\left[\mathrm{R} \$ \times 10^{6}\right] \quad 90$

7.15 Stored Energy Quality Indexes 92 
The major problem - one of the major problems, for there are several - one of the many major problems with governing people is that of whom you get to do it; or rather of who manages to get people to let them do it to them.

To summarize: it is a well-known fact that those people who must want to rule people are, ipso facto, those least suited to do it. To summarize the summary: anyone who is capable of getting themselves made President should on no account be allowed to do the job.

Douglas Adams, The Restaurant at the End of the Universe. 


\section{Introduction}

\section{1 \\ Motivation}

The Brazilian hydrothermal operation planning is centralized by an Independent System Operator (ISO), and it is done with a chain of computational models [1], [2] that uses the Stochastic Dual Dynamic Programming (SDDP) methodology [3]. These models simulate the future behaviour of the system under many hydrological scenarios and calculate a policy that minimizes a risk-adjusted cost-based objective function. The main results of the operation planning studies are weekly thermal generation goals, sub-markets marginal $\operatorname{costs}^{1}$ and solution quality indexes that reflect the expected energy supply as well as the system conditions for up to five years ahead. These results impact the whole energy sector in Brazil, from energy trading contracts to Federal Government policies for energy and economy sectors.

The marginal cost is the smallest cost to produce the next MWh of energy, considering different possibilities of future resource availability, is also used as a basis to set short-term energy prices. These prices, also known as PLD (Portuguese translation of Differences Settlement Price) in Brazil, are used to settle the differences between contracted and generated energy, i.e., the exposure of a market agent, and could possibly be a benchmark for the energy price in bilateral contracts. Although in real applications there may be minor differences in the problem marginal cost and the settlement price (PLD), in this work the only difference between them is that the PLD is limited by both a cap and floor price.

The electrical system expansion planning also uses the marginal costs as economic signals, and requires a stable behaviour and a strong bond between the model marginal cost and the system physical conditions. However, these signals can be inadequate due to the volatility of spot market price. As an example, the occurrence of favourable hydro conditions can decrease spot prices even if there are structural problems with supply [4]. As a consequence, the spot price would rise sharply only when the system is close to a power crisis, when there's no more time to make investments [4]. An investor, the one responsible

\footnotetext{
${ }^{1}$ Lagrange multipliers of the stochastic model
} 
for making the expansion planning become reality, or even an energy trader, may desire regulatory stability and a good predictability of future prices. The system operator, on the other hand, additionally to cost expectation and risk minimization may desire that the decisions, such as thermal generations, are stable and do not change due to small changes in the problem's initial conditions. In all cases, a high variability in either the solution or in marginal costs may increase the future uncertainty and has negative consequences, such as unpredictability of thermal plant fuel requirements that results in higher fuel contract prices, lower future price predictability, increased risk to the investors, traders and hydro generators, fragility of the system operator decisions, lack of a realistic benchmark price and distorted signal to the expansion planning.

Moreover, it is known empirically that the solutions of the SDDP applied to hydrothermal operation problem are more sensible to changes in past inflows, which gives an uncertain information about future resource availability, than to initial condition of other state variables. This fact was already pointed out in [4] and was called "hydrological noise" in that work. The solution variability and its impacts are commented with more details in [4] and [5]. This behaviour is often criticized by market agents, as it increases their business risk and decreases the attractiveness of the energy sector, and a technique to regularize the solutions is being sought for a long time. The causes of such behaviour are related to several aspects of the problem, from modelling and models usage to physical characteristics of the system, and are going to be discussed in details in Section 3.3. Some mechanisms, such as bilateral energy contracts, can provide an adequate hedge against spot price volatility for thermal plants, but in the case of hydro generators (majority of Brazilian generation capacity) these contracts might not be sufficient to provide a complete hedge and agents can be forced to transact substantial amounts of energy in the spot market, which might disrupt their revenue streams depending on the spot prices [5].

\section{2}

\section{Objective}

The main objective of this work is to propose a modification on the formulation of the long term power generation planning problem aiming at reducing decisions and marginal costs variability without compromising the desired solution properties given by the standard modelling The second objective is to propose indexes to assess the solution quality, allowing the comparison of several case studies.

In this work we present two proposals aimed at reducing the solution 
variability:

1. The first one reduces thermal generation variability by modifying the problem formulation penalizing its variation over time, and makes use of an increased state space for the problem.

2. The second one reduces both thermal generation and marginal costs variability by the reduction of the information used to calculate future decisions, and requires two modifications on the standard SDDP methodology:

- the use of different time series models for the forward and backward procedures; and

- the reduction of the state space of the problem.

This proposal makes use of a serially independent approach to model the stochastic process in the backward step of the algorithm and a more conservative risk aversion parametrization, and envisions a cost-to-go function that is less sensitive to variations in the inflows stochastic values.

\section{3}

\section{Contributions and Work Organization}

Although hydrothermal planning problem solution variability and its drawbacks have been previously identified in the literature, actually we could not find any reference to a stabilization technique for multistage problems within the SDDP framework. With respect to the existing literature, the contribution of this work is to propose two approaches that stabilizes the problem solutions while preserving its desirable characteristics, such as the security of the system, among others. A more stable formulation approach and less dependent on current conjuncture and uncertain near future information increases future prices predictability and, as a consequence, reduces the agent's risks, makes the spot prices more stable and closer to become an energy price benchmark, and improves the quality of the operation price signal received by the expansion planning.

A second contribution of the work is the proposal of several indexes to compare solutions of hydrothermal operation planning problems, which makes the comparison between different solutions and methodologies more structured and objective.

As a by-product of the proposed approach, which is possible to be seen as a third contribution of this work, we show that it is possible to use different time series models in the SDDP forward and backward steps and still get good 
solutions. This opens the possibility for many applications that cannot use the SDDP algorithm nowadays, as it is possible to sample states for the SDDP in the forward step of the method using any time series model, even nonlinear ones, and solve the problem with simpler models in the backward procedure.

The remainder of the document is organized as follows: Section 2 describes the notation used in this work. Section 3 provides an overview of the Brazilian hydrothermal operation problem framework, discuss the solution variability within this problem and shows some results that corroborates the motivation of this work. Section 4 describes briefly the SDDP algorithm with time dependency and shows that the consideration of a time series model such as an autoregressive leads to an increase in the solution variability due to propagation of initial conditions to the future by the time series models. In Section 5 we propose and discuss the use of two different modelling options of the problem aiming at reducing the solution variability. Section 6 proposes several indexes to assess quality and compare different solutions of hydrothermal planning problem. In Section 7 we present a case study based on real data for the Brazilian system and compare proposed approaches with the standard one, and Section 8 contains some general conclusions and future work. 


\section{Notation}

In this section we define the notation used throughout the document. Vectors are denoted by lowercase letters, while matrices use capital letters. The stage index is shown as a subscript, as in vector $x_{t}$ or in matrix $A_{t}$. We assume a discrete-time framework in which the system is evolving. The state variables for a given stage $t$ are known and denoted by $x_{t-1}$. Moreover, we assume a linear state-transition function of the form $A_{t} x_{t}=b_{t}-B_{t} x_{t-1}$. Such relation defines the state transformation from one stage $t$ to the next $t+1$.

The dynamic programming equation of a given stage $t$ receives as inputs the state vector, $x_{t-1}$, and the realization of the uncertainties for the current stage, $\xi_{t}$, and returns a measure for the impact of optimal decisions from such stage until the end of the problem horizon: $Q_{t}\left(x_{t-1}, \xi_{t}\right)$. In this setting, the corresponding dynamic programming equation of stage $t$ can be defined as the following optimization problem:

$$
\begin{aligned}
Q_{t}\left(x_{t-1}, \xi_{t}\right)=\min _{x_{t}} & c_{t}^{\top} x_{t}+\mathcal{Q}_{t+1}\left(x_{t}\right) \\
\text { s.t. } & A_{t} x_{t}=b_{t}\left(\xi_{t}\right)-B_{t} x_{t-1} \\
& x_{t} \geq 0,
\end{aligned}
$$

where $x_{t}$ is the decision vector of stage $t$, and $c_{t}$ the objective function cost vector. The constraints are written using matrices $A_{t}$ and $B_{t}$, and vector $b_{t}$, with $B_{t}$ being the stage coupling coefficients matrix. We also define the expected cost-to-go function as $\mathcal{Q}_{t+1}\left(x_{t}\right)=\mathbb{E}\left[Q_{t+1}\left(x_{t}, \xi_{t+1}\right)\right]$, where the expectation $\mathbb{E}[\cdot]$ is always taken with respect to the uncertainty parameter $\xi_{t+1}$, which is assumed to have known probability distribution. The recourse function $Q_{t+1}$ is evaluated over the states variables $x_{t}$ and the uncertainty realization $\xi_{t+1}$.

Any of the problem parameters, $A_{t}, B_{t}, b_{t}, c_{t}$, may depend on the uncertainty realization $\xi_{t}$. In this work we assume that only $b_{t}=b_{t}\left(\xi_{t}\right)$ is uncertain, that is, depends on the realization of $\xi_{t}$ while $A_{t}, B_{t}, c_{t}$ are considered deterministic. In some cases, only part of $b_{t}$ is assumed to be random, and when this is the case, it is going to be properly emphasized along the text. The considered approach to solve the problem is to use the SDDP algorithm applied to the sample average approximation (SAA) problem, as defined in [6]. From the original probability distribution, we randomly draw a sample of size $N_{t}$ and 
denote $\hat{\xi}_{t} \in\left\{\xi_{t}^{1}, \cdots, \xi_{t}^{N_{t}}\right\}$, with $\xi_{t}^{i}$ being its $i$-th sampled value. For notation simplicity, we denote $b_{t}^{i}=b_{t}\left(\xi_{t}^{i}\right)$. The original problem (2-1) is replaced by the sample average approximate problem

$$
\begin{aligned}
\widetilde{Q}_{t}\left(x_{t-1}, \xi_{t}^{i}\right)=\min _{x_{t}} & c_{t}^{\top} x_{t}+\widetilde{\mathcal{Q}}_{t+1}\left(x_{t}\right) \\
\text { s.t. } & A_{t} x_{t}=b_{t}^{i}-B_{t} x_{t-1} \\
& x_{t} \geq 0,
\end{aligned}
$$

where $\widetilde{\mathcal{Q}}_{t+1}\left(x_{t}\right):=\frac{1}{N_{t+1}} \sum_{i=1}^{N_{t+1}} \widetilde{Q}_{t+1}\left(x_{t}, \xi_{t+1}^{i}\right), \forall t \in\{1, \ldots, T-1\}$, with $T$ being the total number of stages, and $\widetilde{Q}_{T}=0$ by definition.

The SAA problem (2-2) is solved using the SDDP algorithm. For simplicity, and without loss of generality, we are going to assume that at each stage and iteration only one sample is taken from the probability distribution of $\xi_{t}$ in the forward step of the algorithm. Thereby, the approximate $t$-stage problem after iteration $k$ of the SDDP algorithm may be written, for a given scenario $\xi_{t}^{i}$, as:

$$
\begin{array}{cl}
\min _{x_{t}} & c_{t}^{\top} x_{t}+\mathfrak{Q}_{t+1}^{k}\left(x_{t}\right) \\
\text { s.t. } & A_{t} x_{t}=b_{t}^{i}-B_{t} x_{t-1} \\
& x_{t} \geq 0 .
\end{array}
$$

The current approximated expected recourse function, denoted by $\mathfrak{Q}_{t+1}^{k}\left(x_{t}\right)$, is given by the maximum of a collection of cutting planes, and can be defined recursively as $\mathfrak{Q}_{t+1}^{k}\left(x_{t}\right)=\max \left\{\mathfrak{Q}_{t+1}^{k-1}\left(x_{t}\right), \widetilde{\mathcal{Q}}_{t+1}\left(x_{t, k}^{*}\right)+\widetilde{g}_{t+1, k}^{\top} \cdot\left(x_{t}-x_{t, k}^{*}\right)\right\}$, where $x_{t, k}^{*}$ is the trial decision used to estimate $\widetilde{\mathcal{Q}}_{t+1}\left(x_{t}\right)$ and $\widetilde{g}_{t+1, k}$ is a subgradient of $\widetilde{\mathcal{Q}}_{t+1}$ at trial decision $x_{t, k}^{*}$. 


\section{Overview of the Brazilian Hydrothermal Operation Planning}

\section{1}

\section{Physical characteristics}

The Brazilian interconnected power system, the largest in Latin America, had an installed capacity at the end of 2012 of 100,217.00 MW (plus 6,275 MW available from Paraguayan part of Itaipu hydro plant), from which $79.8 \%$ comes from hydro plants, $18.8 \%$ comes from thermal plants, and $1.4 \%$ from wind and biomass [7]. The energy production during 2012 was 513,184.5 GWh, from which hydro plants accounts for $86 \%$ and thermal plants for $12.4 \%$ [7].

The hydro system is composed of several reservoirs and of run-of-the-river plants. They are arranged in a complex topology over several basins and together have a multi-year regularization capacity. Thermal generation includes nuclear, natural gas, coal and diesel plants. These different power sources are distributed around the country and are interconnected by $106,443.7 \mathrm{~km}$ of high voltage (equal or above $230 \mathrm{kV}$ ) transmission lines grid [7]. Figure 3.1, taken from [7], shows the most important transmission lines and substations connected to the basins of Brazilian National system. Regarding transmission grid characteristics, Brazil can be divided in four sub-systems: South-East (SE), which also comprises Central-West region, South (S), North-East (NE) and North $(\mathrm{N})$.

\section{2}

\section{Operation Planning Overview}

In Brazil, the electrical system operation is centralized in an independent system operator, a non-profitable private company called ONS (Operador Nacional do Sistema Elétrico, which means Electrical System National Operator). This company is responsible not only for controlling the system operation, but also for planning the operation and assessing system's security conditions for next years. The main objective of the operation planning problem is to optimize the system operation for a cost-based criterion, considering the future conditions of the system, such as resources availability, energy demand and physical constraints, among others. Due to Brazilian hydro plants multi-year regularization capacity, the planning horizon comprises up to 60 months (5 


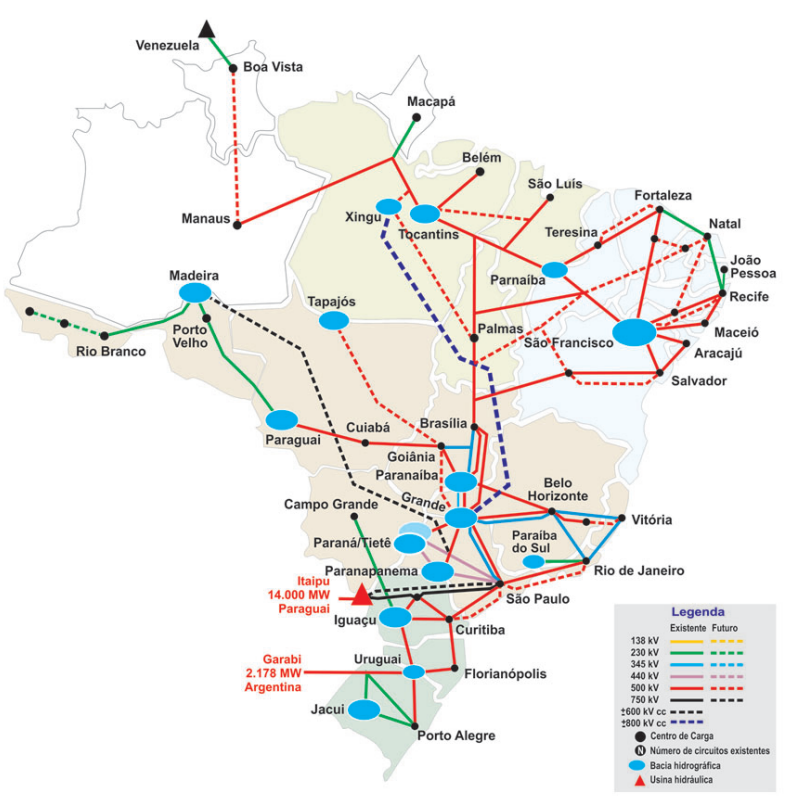

Figure 3.1: Brazilian interconnected power system

years). In such a long horizon, the level of details needed by system operator is different depending on the period, e.g., the closer to the current date the more detailed the information should be. In order to consider more details in the solution of such short-term period, the complexity of the modelling process, in terms of physical system representation, is higher than for long-term periods. On the other hand, the uncertainty related to the problem's variables increases when we move away from current date, and a different type of complexity arises, this time not related to the physical system modelling, but to uncertainty modelling. In order to deal with such a diverse problem, which requires an intense computational effort to be solved, a decomposition approach was used and the problem is solved by several models, as described in [1]. The main idea of this approach is to use different models for the short-term, in which the physical representation of the problem is better detailed, and for the medium-term, in which the uncertainties of the problem are modelled but the physical representation is sacrificed.

In the next sections we are going to discuss the main idea behind each solution strategy, and pave the way to discuss the main idea of this work, the solution variability reduction.

\subsection{1}

\section{Medium-term problem}

The medium-term horizon problem comprises up to five years ahead (discretized monthly), and is responsible to give the system operator information regarding future cost, thermal generation expectations, possibility of energy 
supply shortages in the future, among others. These results allow the operator to decide which measures should to be taken to avoid having any problems with the demand supply during the next years.

The first issue that arises in the medium-term problem is how to solve the problem in such a way that the model considers it as an infinite horizon problem, and not a finite 60 months problem. In order to obtain a reasonable boundary condition that represents the continuity of the energy supply after the planning horizon, a common practice is to consider 60 additional stages in the problem. Hence, the objective function of the planning problem is to minimize a measure of the operation cost (expected value or convex combination between expected value and a risk measure) along the up to 120 stages of the problem, while supplying the area loads and obeying technical constraints. The total cost in one stage is the sum of thermal generation costs plus a penalty term that reflects energy shortage.

By the nature of the results needed for the medium-term horizon and the computational burden a detailed representation of the physical system would certainly bring, a simplification of the problem was adopted in this horizon. In order to reduce the problem dimension, the hydro plants in regions with similar hydrological behaviour are aggregated in equivalent energy reservoirs, as proposed by [8] and [9]. This representation considers hydro plants with reservoirs separately from run-of-river plants, as in the second ones there is no decision regarding the water storage. As a consequence of this aggregate representation, the associated measure units are represented as stored energy and energy inflows. Some systems are connected to each other by transmission lines, which are represented as a single equivalent connection. The interconnected Brazilian system can be represented by four generation nodes - comprising sub-systems South-East (SE), South (S), North-East (NE) and North $(\mathrm{N})$ - and one transshipment node called Imperatriz (see Figure 3.2).

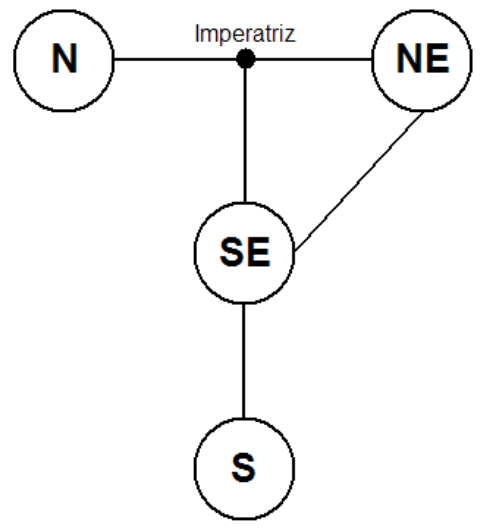

Figure 3.2: Brazilian interconnected power system representation 
Although this approach is a simplification of the original problem and a reduction in its size, many characteristics of the original problem are still represented. As any hydro plant, the resulting energy equivalent systems are subject to generation and storage capacity, storage availability and uncertain energy inflows. Moreover, as it happens with any individual hydro plant, the energy production efficiency of the equivalent systems also decreases when the storage level decreases. Energy losses due to evaporation are also considered, and increases proportionally to the stored energy in the reservoirs. The details of how the equivalent energy modelling was implemented in the models used by the Brazilian system operator are found in works such as [10], [11], [12] and [13].

Despite the monthly discretization of the problem, the official modelling considers three load levels within the month in order to represent difference of the demand level in different times of the day, such as the peak hours.

Regarding thermal plants, they are represented individually and consider a minimum and maximum generation capacity, but are not subject to fuel availability constraints in the current formulation. Unlike hydro plants, thermal plants have a production cost per energy unit, represented by fuel costs in the problem formulation.

In summary, the medium term hydrothermal operation planning problem is a cost expectation minimization, in which the decision variables are associated with equivalent systems and thermal plants dispatch and with energy exchanges between systems. The only uncertainty represented in the problem is related to the inflows to the equivalent systems. The inflows distribution at each stage is modelled and, in order to allow the problem to be solved numerically a finite number of scenarios is sampled from these probability distributions. In this step, problem becomes deterministic, as the probability distributions are replaced by the sampled scenarios tree. Although the number of energy systems is small, the total number of possible states for the problem grows very fast with the number of scenarios sampled at each stage. In order to solve this problem, an SDDP-based model is used. This method is capable of solving large scale problems due to the fact that, instead of using the complete scenarios tree, it samples subsets of it at each iteration. As a consequence, the solution of the algorithm - if stopped before the optimality - is random. Indeed, as the hydrothermal operation planning problem is too large to be solved in reasonable CPU time, it will always be stopped before optimality. The SDDP algorithm is based on Benders decomposition, and solves the problem by iteratively computing functions that couples each stage of the problem. These functions represent, at a given stage, a lower approximation of the ex- 
pected future cost function, and are often called cost-to-go functions. As the iteration number advances, more cuts are added to the cost-to-go functions, which become closer to the real (and unknown) future cost functions and the solution gradually gets closer to the optimal one. Several works give details about the SDDP method, such as [3], [6] and [14]. Work [13] also provides a detailed view of the SDDP algorithm and its application to Brazilian problem.

In order to model the inflows uncertainty and therefore be able to obtain any subset of a theoretical ${ }^{1}$ scenarios tree, a statistical model needs to be used. In the energy planning problems in which hydrological uncertainty is strong, linear regression models are often used to model river flows and energy reservoir inflows. In Brazilian case a periodic autoregressive (PAR) approach is used to model the inflows behaviour, and the scenarios are generated using a combination of a PAR model and a log-normal noise term that guarantees non-negative inflows values. This approach is described in general terms in the works [15], [16], [17] and with more details regarding the implementation used in Brazil in works [18], [19], [20], [21] and [13].

The main characteristic of the autoregressive modelling applied to inflows is that future conditions of the systems are going to be conditioned by the past inflows values. This behaviour is desirable, at least in principle, in the sense that, if there is any sort of correlation in the historical data, this will also be true for the generated scenarios. In Brazilian medium-term modelling the past inflows information of up to six months is used to generate the monthly scenarios for up to five years ahead (plus five years post planning horizon for boundary conditions), and a scenarios tree is generated using 20 random noises per stage, including the first one, which is not considered deterministic in this planning horizon.

\subsection{2}

\section{Short term problem}

The short-term problem, in Brazilian hydrothermal system operation planning, comprises up to two months. Unlike the medium-term problem, that considers a longer planning horizon and then sacrifices the physical representation of the system in order to better model the uncertainty, the short-term problem is able to model the physical characteristics of the problem with more details. Although computational power is not a limitation for this problem nowadays, the uncertainty of the inflows is not considered in the first month, which is discretized in weekly intervals. The weekly inflows forecasts

\footnotetext{
${ }^{1}$ The use of the word theoretical is just to emphasize that the complete scenarios tree is never totally sampled, as it would require a large amount of memory and could easily become computationally impracticable.
} 
are computed using several models, depending on the forecast horizon and the hydrological post in question. In summary, the first week forecast for the most of the important posts are calculated using a rainfall-run-off model called SMAP (Soil Moisture Accounting Procedure) [22] that take not only past inflows into account, but also current soil conditions combined with observed and forecast rain. The first week forecasts for the remaining posts, such as the rest of the month forecast for all posts, are calculated using autoregressivemoving average family models, and consider only inflows information. In the second month, on the other hand, the uncertainty is considered by sampling monthly scenarios using PAR models similarly as done in the medium-term problem.

Unlike the medium-term problem that is solved by a sampling based decomposition algorithm (SDDP), the short-term problem is solved using a deterministic decomposition method, the L-Shaped [23]. The main difference between both methods is that, unlike the SDDP that samples subsets of the complete scenarios tree and obtain a random solution, the L-Shaped method solves the entire scenarios tree and returns the optimal solution (given an optimality gap). This method is only possible to be applied to the problem due to its reduced dimension (two months horizon).

Although the short-term planning horizon ends in the second month, the future cost function for the second month, calculated during the medium-term problem solution, is used as a boundary condition and is added as a set of constraints to the second stage problems of the solution algorithm. The main consequence is that the decisions taken in the short-term problem consider its impact up to five years ahead, and not only within the two months horizon.

\subsection{3}

\section{Risk aversion}

Although used for many years in Brazil and still nowadays in many countries, the risk neutral version of SDDP algorithm can lead, in some situations, to critical states of the system and even to energy shortages. A possibility to improve SDDP results for hydrothermal operation planning problem is to resort to a risk averse implementation of SDDP. The probability of achieving such critical states is reduced when some type of risk aversion is used within the SDDP algorithm. Shapiro [6] proposes the introduction of CV@R [24] in the SDDP in his theoretical work. The first numerical results of this proposal using an implementation that required the use of additional state space variables is found in [25], which was made available on-line in December 
$2010^{2}$. Later on, another way of implementing the same approach in the SDDP is published in [26], also making use of an increased state space. In January 2012 a simpler approach to consider the CV@R within the SDDP without resorting to the use of additional state space variables was made available on-line $^{3}$ and published later in [14]. In the meanwhile, an application of a direct approach in the Brazilian official model was presented in [27] and [28], and later a real case application to Brazilian problem in [29]. All these works show that, depending on the parametrization chosen, the probability of critical states of the system is reduced and thus its security is improved. In this work, the risk averse approach implemented in [14] is used. There are several other ways to consider risk averse behaviour of the solution in hydrothermal planning problems, but this is out of the scope of this work.

Let the equation (3-1) be the $t$-th stage of our multistage stochastic optimization problem, for $t=1, \cdots, T$.

$$
Q_{t}\left(x_{t-1}^{*}, \xi_{t}\right)=\left\{\begin{array}{cl}
\min _{x_{t}} & c_{t}^{\top} x_{t}+\rho_{\lambda}\left(Q_{t+1}\left(x_{t}, \xi_{t+1}\right)\right) \\
\text { s.t. } & T_{t} x_{t-1}^{*}+A_{t} x_{t}=b_{t} \\
& x_{t} \geq 0
\end{array}\right.
$$

where

$$
\rho_{\lambda}\left(Q_{t+1}\left(x_{t}, \xi_{t+1}\right)\right)=(1-\lambda) \cdot \mathbb{E}\left[Q_{t+1}\left(x_{t}, \xi_{t+1}\right)\right]+\lambda \cdot C V @ R_{\alpha}\left(Q_{t+1}\left(x_{t}, \xi_{t+1}\right)\right)
$$

is the risk measure that is responsible to consider the trade-off between the risk neutral expectation value and the $\mathrm{CV} @ \mathrm{R}$ value. This risk measure takes two parameters into account: $\alpha$ is the percentage of the most expensive scenarios that are used to estimate the CV@R, and $\lambda \in[0,1]$ is a risk aversion parameter, i.e., the CV@R weight in the objective function. The solution of this problem within the SDDP framework is straightforward and is described in details in work [14].

\subsection{4}

\section{Problem Formulation}

As stated before, the objective function of the hydrothermal operation planning problem is to minimize a risk adjusted operation cost along the up to $T$ stages of the problem, while supplying the area loads and obeying technical constraints. Note that the demand supply equation is always feasible (i.e., the problem has relatively complete recourse) due to the inclusion of a dummy thermal plant with generation capacity equal to the demand and operation

\footnotetext{
${ }^{2}$ http://www.optimization-online.org/DB_HTML/2010/12/2861.html

${ }^{3}$ http://www.optimization-online.org/DB_HTML/2012/01/3307.html
} 
cost that reflects the social and economic costs of not meeting the energy demand (deficit cost). The problem can be written, for all $t=T, \ldots, 1$ as:

$\widetilde{Q}_{t}\left(v_{t-1}, \xi_{t}\right)=\min _{x t} c t_{t} \cdot g t_{t}+\sum_{s=1}^{N S} c d \cdot d_{t, s}+\beta \cdot \mathfrak{Q}_{t+1}$

s.t.

$v_{t}+g h_{t}+s_{t}=v_{t-1}+a_{t}$

(Hydro balance)

$v_{t} \leq \bar{v}_{t}$

(Storage limits)

$g h_{t} \leq \overline{g h}_{t}$

(Hydro Generation limits)

$\underline{g t}_{t} \leq g t_{t} \leq \overline{g t}_{t}$

(Thermal Generation limits)

$g h_{t, s}+\sum_{j \in N T_{s}} g t_{t, j}+n f_{t, s}+\sum_{i=1}^{N D} d_{t, s, i}=D_{t, s}$,

$$
\forall s=1, \ldots, N S
$$

(Demand supply)

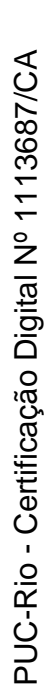

$n f_{t, s}=\sum_{l=1}^{N S}\left(f_{t, l, s}-f_{t, s, l}\right), \forall s=1, \ldots, N S$

(Net Energy flow)

$\left|f_{t, s, l}\right| \leq \bar{f}_{t, s, l}, \forall s=1, \ldots, N S ; \forall l=1, \ldots, N S$

(Energy exchanges limits)

The problem indexes, shown as subscripts in the formulation, are:

$t$ stage identifier;

$s$ system index;

$j$ thermal plants index;

$i \quad$ deficit depth identifier;

$l$ alternative index used to identify the system number.

The variables and parameter dimensions are:

$N T$ total number of thermal plants in the problem;

$N S$ number of systems;

$N D$ total number of deficit depths.

The problem variables are: 
$v_{t} \quad$ vector $[N S \times 1]$ of stored volumes in each system at stage $t$;

$g t_{t} \quad$ vector $[N T \times 1]$ of thermal generations $g t_{t}=\left(g t_{t, 1}, \cdots, g t_{t, N T}\right)$;

$d_{t, s} \quad$ vector $[N D \times 1]$ of energy deficits in system $m$ in all $N_{d}$ depths, as shown in $d_{t, s}=\left(d_{t, s, 1}, \cdots, d_{t, s, N D}\right)^{\top}$;

$g h_{t} \quad$ vector $[N S \times 1]$ of hydro generations $g h_{t}=\left(g h_{t, 1}, \cdots, g h_{t, N S}\right)^{\top}$;

$s_{t} \quad$ vector $[N S \times 1]$ of spilled energy in each system;

$g h_{t, s} \quad$ s-th element of $g h_{t}$ vector, denotes the hydro generation in system $s$;

$g t_{t, j} \quad$ j-th element of vector $g t_{t}$, denotes the generation in thermal plant $j$;

$n f_{t, s}$ net energy flow of system $s$ on stage $t$;

$d_{t, s, i} \quad$ i-th element of vector $d_{t, s}$, denotes the deficit in depth level $i$;

$f_{t, l, s}$ energy exchange [scalar] between systems $l$ and $s$ on stage $t$;

The problem constants are:

$c t_{t} \quad$ vector $[1 \times N T]$ of unit generation cost of thermal plants;

$c d \quad$ vector $[1 \times N D]$ of unit deficit costs $c d=\left(c d_{1}, \cdots, c d_{N_{d}}\right)$;

$\beta$ discount factor [scalar] used to calculate the present value of expected future cost;

$a_{t} \quad$ vector $[N S \times 1]$ of energy inflows in systems equivalent reservoirs;

$\bar{v}_{t} \quad$ vector $[N S \times 1]$ of energy storage capacity in each system;

$\overline{g h}_{t} \quad$ vector $[N S \times 1]$ of maximum hydro generation for each system;

$\underline{g t}_{t} \quad$ vector $[N T \times 1]$ of minimum thermal generations;

$\overline{\overline{g t}}_{t} \quad$ vector $[N T \times 1]$ of maximum thermal generation for each plant;

$N T_{s}$ set of indexes of thermal plants of system $s$;

$D_{t, m}$ energy demand on system $m$ on stage $t$;

$\bar{f}_{t, s, l}$ energy exchange [scalar] capacity from system $s$ to $l$.

The approximation of the recourse function, $\mathfrak{Q}_{t+1}$, denotes the approximated expected future cost function in risk neutral cases, but can also denote the convex combination of the expected future cost function with a risk measure of the future cost distribution.

Although simplified in terms of physical representation of the system, the presented formulation preserves the main characteristics of the problem and its large scale size, which is important in order to evaluate the proposed approaches. The physical modelling used in this work is similar to the one used in work [14].

It is important to mention that in real applications several details are modelled in order to better represent the physical behaviour of the system, even in the medium term problem (in which the hydro plants are modelled as equivalent reservoirs), such as the existence of run-of-river plants, the energy production coefficient changes with respect to storage levels, losses due to 
evaporation and different load levels to roughly represent peak and off-peak hours of the month. In this work all these characteristics are not modelled, as the main points of interest for this work remain present in the simplified approach.

\section{3 \\ Solution variability}

The excessive solution variability of Brazilian energy operation planning is being discussed for a long time. Since 2006 it is possible to find references, such as $[30]^{4}$, that proposes several changes in order to stabilize the models solutions. This work in particular suggests that, instead of using a weekly price as currently used in Brazil, an average monthly price would be more stable. Although this is naturally true, the cause of the variability was not discussed in this document. In the same line, several proposals were presented in the energy sector events in 2008, mainly focused on calculating the energy spot price as an average of past and forecast prices $^{5}$ given by the optimization models used for the operation planning. A weekly price calculated by a moving average of the prices of the last five weeks was almost implemented regarding sources that published, in 2009, an interview given by the Brazilian System Operator director ${ }^{6}$. In the same year, in the event Energy Trading Brasil 2009, a representative of the system operator gave a speech ${ }^{7}$ in which five measures to deal with the price volatility were announced:

1. Inflows time dependence attenuation in the cost-to-go function:

The idea behind this proposal was to consider smaller numbers in the inflows autoregressive coefficients during the cost-to-go function calculation, in an attempt to reduce the autoregressive models importance in the medium-term problem. This approach is the closer to this work.

2. Anchorage of the weekly forecasts:

This proposal consists of - instead of letting the weekly forecasts vary depending on additional informations (such as rain forecast) - averaging the weekly forecast with a monthly forecast made in the beginning of the

${ }^{4}$ Document sent to Brazilian's energy sector regulator (Aneel) public hearing AP 18-2006, available on-line at (in Portuguese): http://www.aneel.gov.br/aplicacoes/audiencia/arquivo/2006/018/contribuicao/duke_energy_pld_mensal_carlos_dornellas.pdf

${ }^{5}$ See the presentation available on-line at (in Portuguese): http://www.abce.org.br/xivsimposio/downloads/roberto_castro.pdf

${ }^{6}$ Details can be found on-line at (in Portuguese): http://www.cerpch.unifei.edu.br/not01.php?id=3129

${ }^{7}$ Presentation available at the ONS website: http://www.ons.org.br/download/biblioteca_virtual/palestras/2009_05_25_Energy_Trading.pdf 
month. The idea is that the resulting forecast was going to be more stable and, therefore, the resulting prices. Although this proposal could have some variability reduction within each month, the variability between months would increase. Moreover, the idea of combining two forecasts in an unsound methodological way just to avoid solution variability was not well received by the sector.

3. Consideration of the uncertainty of the weekly inflows:

This proposal consists of generating scenarios for the weekly stages of the first month instead of solving the problem as if the first month was deterministic. This approach, although has never been implemented in the official model, has the potential to reduce the variability caused by the coupling between the SMAP model, used in the first week, with the Box \& Jenkins models used in the other weeks.

4. Selective sampling of the scenarios:

This was the only proposal implemented in the official model, although the results were not as good as initially expected with respect to the solution and price variability. It basically changes the way the noises to generate the scenarios are sampled. First, a very large sample is taken from the noises distribution considering all subsystems. Second, this large sample is clustered in a smaller number of noise terms and, with the resulting noises vector, the scenarios are generated. This approach is proposed and detailed in [21].

5. Implementation of resampling of the forward scenarios during the SDDP algorithm solution:

This proposal consists of adjusting the current implementation used in Brazil to resample the scenarios used in the forward step of the algorithm at each iteration instead of using a fixed set during the entire iteration process. This characteristic in the implementation of the SDDP was already described in its proposal [3] and was essential in the proofs of convergence published later, such as [31], [32], [6] and [33], but actually is not going to affect the solution variability as it was believed when this speech was given, in 2009.

In the years that followed the solution variability continued to appear in the news, sometimes only as general complains from various energy sector agents $^{8}$, and sometimes from investors of specific sectors, as occurred in 2012

${ }^{8}$ News published in 2010 in Canal Energia website with the title "Elevação de PLD não indica desabastecimento, mas volatilidade preocupa, se- 
when small hydro plants (SHP) investors reported the prices volatility as one of the sources of the crisis in the SHP sector ${ }^{9}$. It is also possible to find short studies from universities trying to identify the causes of prices volatility in Brazil, such as [34] and [35]. In [34], the main causes identified were the lack of investments in the energy sector, mainly regarding the system expansion through hydro plants without storage capacity, environmental constraints and the system operator operations criteria, which are strongly model based. On the other hand, in [35], the thermal plants and fuel availability constraints are pointed out as the major cause for historical episodes of high marginal cost variability in Brazil. The possible causes for the models solutions variability are many, some already identified in those works, and are going to be discussed in this section.

In an attempt to find relations between Brazilian and other markets, Canal Energia agency even published in 2012 some news comparing Brazilian and European markets ${ }^{10}$ in which prices volatility is criticized. Also in 2012 it was reported by Valor Econômico ${ }^{11}$ newspaper that, in an interview given by the director of ONS, both ONS and CCEE teams were already working on the marginal cost volatility issue to propose improvements to mitigate this problem.

Since then, this topic has come several times to discussion and, in 2014, rumours that changes in Brazilian price formation were imminent were propagated by energy sector ${ }^{12}$. In the end of 2014 ONS's director again talked about the marginal cost volatility reduction ${ }^{13}$, and said that ONS was going to propose during 2015 some changes in the current models in order to reduce their solution variability.

Although, as seen in this section, the solution variability is one characteristic often criticized about the models used nowadays, the current literature identifies some variability sources but fails in proposing improvements to re-

gundo agentes": http://www.canalenergia.com.br/zpublisher/secoes/Principais_Noticias.asp?data $=4 / 3 / 2010$

${ }^{9}$ News published in 2012 in Canal Energia website with the title "Setor de PCH tenta encontrar saídas para crise da fonte": http://canalenergia.com.br/zpublisher/secoes/Principais_Noticias.asp?data=22/03/2012

${ }^{10}$ Published in Canal energia in 2012 with the title "Europa: Mercado livre, transparente e isonômico": http://www.canalenergia.com.br/zpublisher/secoes/Principais_Noticias.asp?data $=04 / 10 / 2012$

${ }^{11}$ Available on-line at: http://www.valor.com.br/brasil/1122814

${ }^{12}$ Some similar news were published by different sources, such as Globo newspaper, available at: http://oglobo.globo.com/economia/petroleo-e-energia/cesp-defendemenor-volatilidade-do-preco-da-energia-de-curto-prazo-1-13735032

${ }^{13}$ This news was published by several sources, one of them available on-line at: http://gasnet.com.br/conteudo/17000/ONS-quer-alteracoes-para-conter-volatilidade-noCMO 
duce the solution variability. In this context, lets first identify some of the main sources of solution variability in the Brazilian Energy Sector:

\section{- Reduced regularization capacity}

One main characteristic of hydrothermal systems strongly hydro based, such as the Brazilian one, is the possibility to regularize the inflows for several years, allowing the system operator to smooth the impact that hydrological variability would have on the system operation. However, during some time in Brazil - specially during the nineties - the lack of investments in large projects stopped the system expansion through the construction of big reservoirs. Moreover, due to the fact that environmental constraints are getting increasing importance in recent years, the construction of big dams are out of the Brazilian expansion plan. The immediate impact of the system expansion only through small projects or big projects by without storage capacity is that the system regularization capacity decreases over time, together with the benefit of smoothing the solution variability due to uncertainty of inflows. As examples, consider two extreme cases: the first one with infinite regularization capacity, and the second one with no reservoirs at all. In the first case, depending on the storage level, it could be possible to supply the demand for several years. In this case, even though the inflows could vary a lot, there would be no need to keep changing the solution from one stage to the other, as the stored water could be used to generate energy in case there was a drought. In the second case, on the other hand, the thermal dispatch would change all the time depending on the inflows, as there is no stored water to be used. In this case there is no decision to be taken, and the solution variability is expected to be very high. As the Brazilian system is moving towards the second example, it is expected that the solution variability increases, as the system operator's ability to regularize the inflows is reducing. This work is not going to focus on this source of solution variability.

\section{- Modelling of river basins with different behaviour within the same equivalent reservoir system}

This topic is mainly related to the medium term problem modelling. When the four systems are modelled into energy reservoirs, it is assumed that the hydrological behaviour of all plants considered within the same system are the same, that is, they belong either to the same basin or to basins with similar behaviours. However, some big projects, such as Itaipu, were constructed in one physical region (in this example, Southern) but are connected directly to other region through long transmis- 
sion lines (in this case, South-eastern region). The main characteristic of Brazilian South region is the erratic behaviour of the inflows, mainly due to the soil type in this region, that allows the inflows to increase very fast, but also to reduce very fast. In this case, the South inflows behaviour impacts directly the Southeast equivalent energy inflows behaviour, as Itaipu plant is considered as part of Southeast system. This characteristic makes the Southeast inflows volatile every time there is a large variation in Itaipu inflows, and then, can possibly increase the solutions variability. This problem is currently being solved by the official model developer, Cepel, in an implementation that allows each system to have different equivalent reservoirs to deal with very different hydrological behaviours.

\section{- Thermal plants characteristics}

Another source of solution variability, in this case totally related to energy prices, is the thermal plants cost/power characteristics. Figure 3.3 shows the total thermal generation availability on May 2015 on the vertical axis, and the cost in Brazilian Reais to produce each MWh (CVU, Portuguese acronym for unit variation cost). The bottom blue are represents the inflexible thermal generation, while the red area represents the dispatchable thermal resources. It is clear that, depending on the current thermal generation, it is possible to vary the thermal dispatch without changing much the marginal cost (the cost regions with high vertical steps in this graph allows big changes in thermal generation without significant changes in marginal cost). On the other hand, thermal availability [MWa] areas with long horizontal steps implies that any change in the thermal dispatch can make the marginal cost very different from its previous value. In practice, as the solution marginal cost can be the water value instead of the CVU of the marginal thermal plant, this effect is smaller in reality, but still exists and, depending on the thermal dispatch, the marginal cost variability can be higher or lower.

\section{- Deterministic approach in the first month and coupling between different inflows forecasting models}

As already mentioned before, the first month of the short-term problem is deterministic and is discretized weekly. The only decision that is actually implemented is the first week one and, in the end of the first week, another problem is solved considering what actually happened during this week as initial conditions and a new forecast for the second week of the month (this time considering some rain forecast information to predict the inflows for this week). The fact that forecasts are never equal to reality by itself introduces some variability to the problem, as 


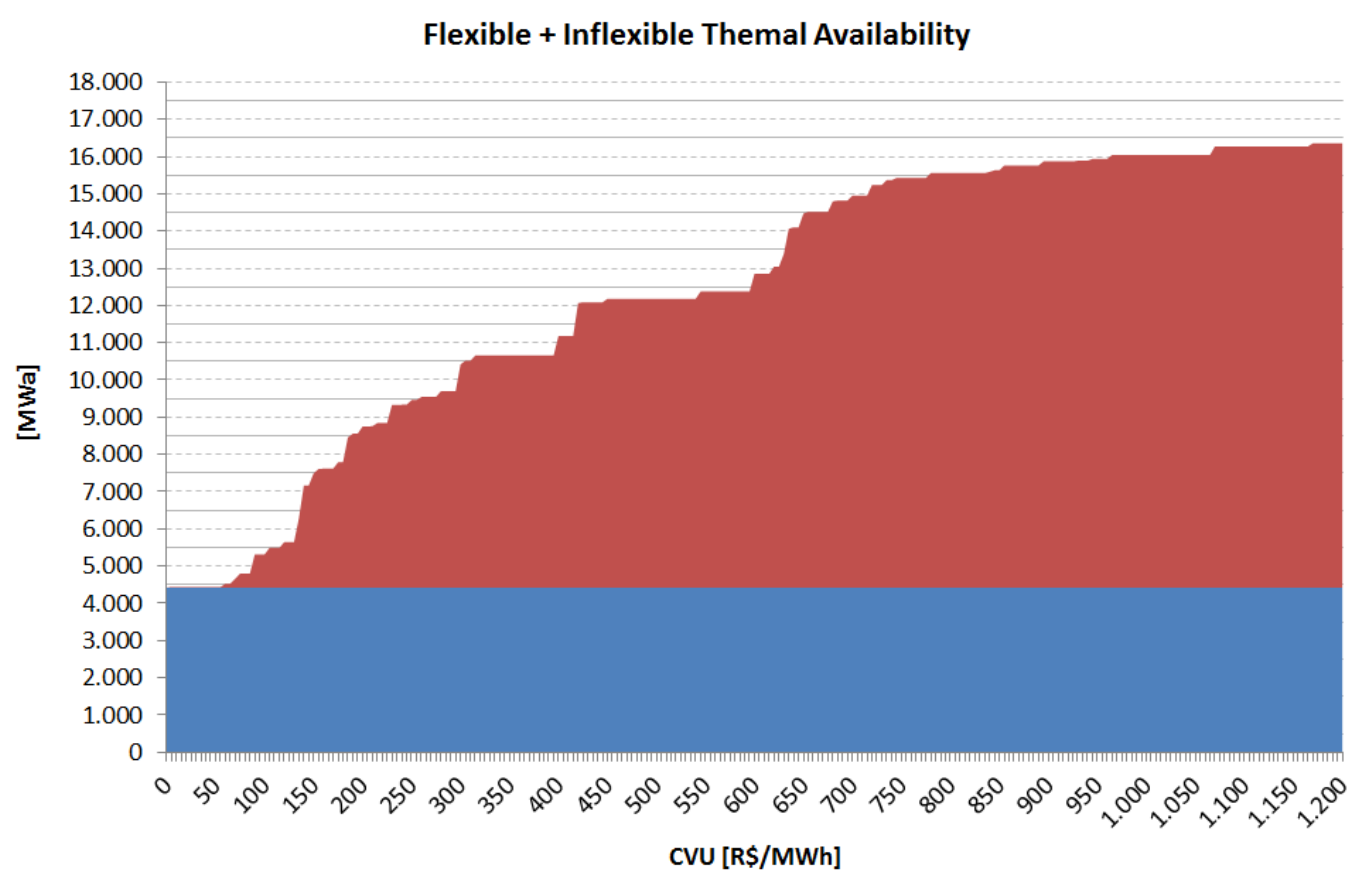

Figure 3.3: Thermal generation availability and unit variation cost - May/2015

the solution taken is never perfect for what is really going to happen. However, this problem can only be reduced with the improvement of the forecasts quality. This is already being done by the system operator with the use of SMAP models in an increasing number of hydrological posts. On the other hand, there is another source of solution variability: the use of Box \& Jenkins models for the rest of the weeks in the first month. The main reason for that increased variability can be understood in the following example: there is a cold front reaching South and Southeast regions in the first week causing the rain forecast models to show strong rains in both regions during the beginning of the first week. The models used to forecast inflows for the first week are going to read the rain forecasts and estimate the amount of water that is likely to become water flows in the rivers which, in this case, will make the inflows increase in both regions. The first week inflows prediction, which is high in this case, is going to be used by models that are, in most cases, autoregressive and are going to propagate this high inflows for the other weeks of the month. However, in the end of the first week the system operator is going to solve a new short-term problem, this time considering what happened in the first week and the second week of the month as the first week of the problem. In this case, the rain-flow models are going to be used again, this time to forecast the second week of the month which may, in this 
case, show no rain forecast for the next ten days. In this situation, the forecast for the second week of the month is going to be dry, the opposite as the previous forecast made by the Box \& Jenkins model for this very same week, and the same will happen to the forecasts for the rest of the month. If we compare the problem that was solved in the beginning of the month, with a high inflows forecast for the whole month (mainly due to a rain in the first week) to the problem solved in the beginning of the second week, with lower inflows for the entire month, we are going to notice an increased solution variability, mainly related to expectation reversals. This problem is also related to the next topic and is going to be discussed later. One possible way to deal with this problem is to consider the weekly uncertainty on the inflows modelling, as this may prevent the decision to be totally biased by a bad forecast, for example, although the entire first month scenarios tree would be biased and so would the decision. One drawback of modelling the weekly uncertainty is that the problem can become too big to be solved by the L-Shaped method and would need a change in the current model used to solve the problem. In work [36] a scenarios tree reduction technique was used to sample scenarios tree that would still allows the short-term problem to be solve by the same method used nowadays.

- Consideration of past inflows in the state space of the problem The last topic to be discussed is the representation of the past energy inflows as state variables in the medium term problem. In this case, what happens is quite similar to what was discussed in the previous topic: the future expectation (given by the cost-to-go function) is biased by the recent events, and as expectation reversals occurs, the next problem will be biased to the opposite direction and can, hence, increase the solution variability. Although expectation reversals are not always happening in the next stage (the models used to generate the scenarios are mainly meant to be used for one-step ahead forecast), as several stages are biased by the first one and the probability that an expectation reversal happens before the model mean reverts is much bigger, we can expect that the solution variability is increased. As an example we show in Figure 3.4 the scenarios generated by the official Brazilian model for January 2015 (in the end of December 2014), together with their average in green, and the performed value in blue. It is clear that expectation reversals occurs even in the first month, although most of times it happens after the first month. The same data is presented for February, and it is possible to see that in this case the scenarios generated are in line with the performed 
energy inflows. The effect discussed in this topic, combined with the

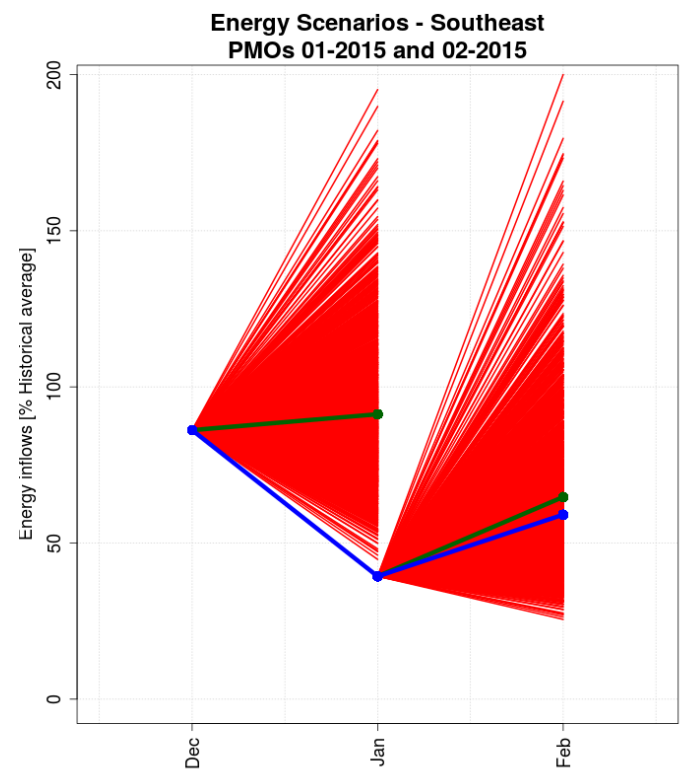

Figure 3.4: Energy inflows in Southeast system as a \% of the monthly historical average

changes in the first stage forecast presented in the previous topic, is one of the major responsible for the solution variability found the operation planning problem in terms of modelling. This is going to be the main focus of this work, and is going to be discussed in more details in the next section.

\subsection{1}

\section{Solution variability with respect to state space variables}

Brazilian hydrothermal operation planning problem decision variables are energy exchanges, thermal generation and hydro plants stored, turbined and spilled volumes over time, while the only uncertainty represented is associated to water inflows in hydro plants. Within a time independent framework, the state of the system at a given stage is described by the stored volumes in the reservoirs at the beginning of that stage. In real applications, however, the inflows to power plants are represented by autoregressive time series models, which requires an augmented state space including past inflows. Although equally represented as state variables, stored volumes and past inflows are different in terms of the information they carry on. While stored volumes accurately represents resources currently available, past inflows helps to predict future resource availability, which is an uncertain quantity. Another characteristic of stored volumes is that it varies smoothly over the time frame considered - at least for most of reservoirs, where water inflow/outflow volumes 
are small compared to storage capacity - while the past inflows show high variations in short periods of time.

Even though one could intuitively think stored volumes should be more important due to certainty of information about resources availability it carries on, the solutions are more sensible to changes on past inflows than to changes on stored volumes, as it will be shown in the ensuing text. As a consequence, an undesirable variability occurs, as shown in Figure 3.5 (plotted with data from [37]). Indeed, it shows Brazilian weekly thermal generations of the SouthEast system from October/2012 to March/2013 (red line plot, left axis). Its percentage variation is shown by the bars (right axis). The mean absolute percentage weekly variation is equal to $10.3 \%$, with a maximum observed value of $40 \%$. This high variability is observed on real data, and cannot be attributed only to inflows variability, but also to the other aspects discussed in this section, specially to the fact that, although the uncertainty in the first month is high, it is modelled by a deterministic approach in the weekly stages.

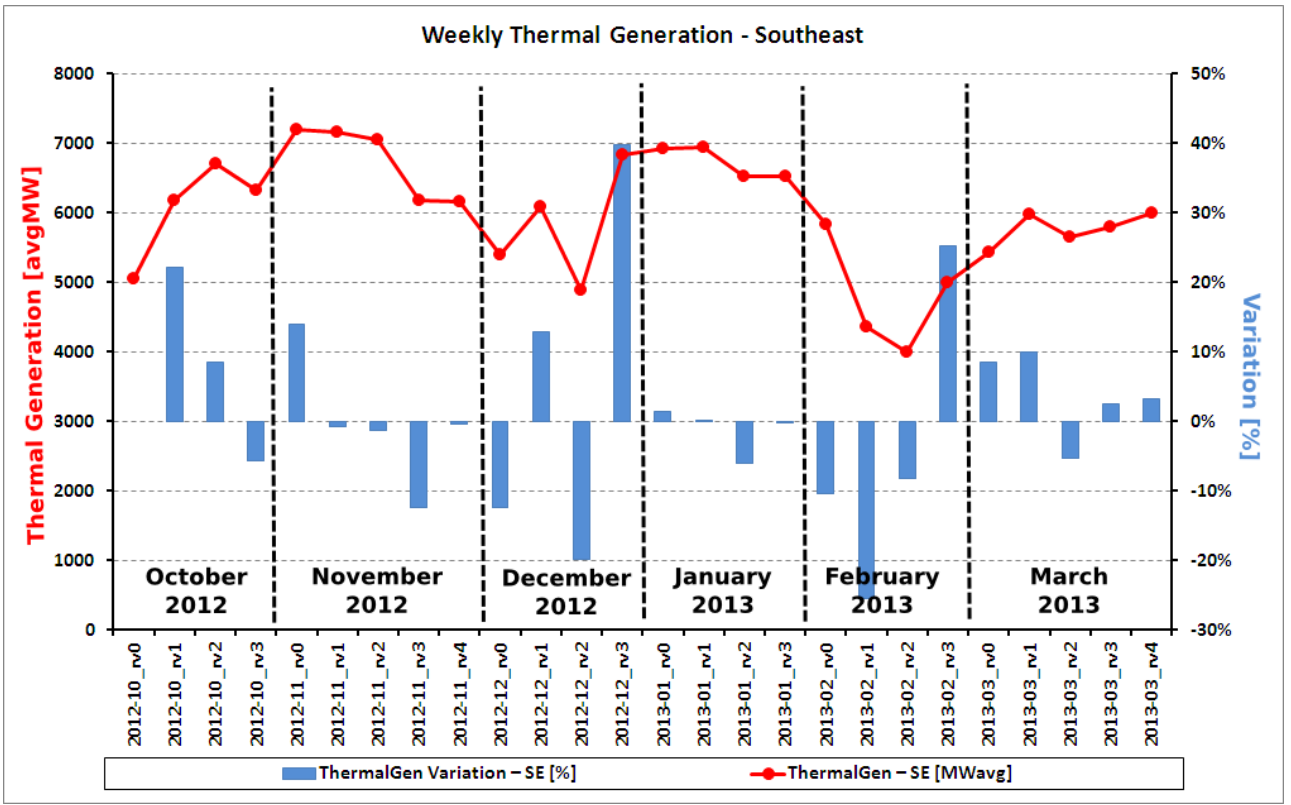

Figure 3.5: Weekly thermal generation and variation - Oct/2012-Mar/2013

Additionally, the high variability on energy prices shown in Figure 3.6 (plotted with data from [37]) also poses a challenge to agents involved in the Brazilian energy market. The energy price is the marginal cost obtained as the Lagrange multiplier (dual variable) of the demand supply equation. Figure 3.6 shows a high variation of marginal costs through weeks: the mean percentage absolute variation is $25.5 \%$. The bars on Figure 3.6 show the changes of the marginal costs (in $\mathrm{R} \$ / \mathrm{MWh}$ ), and its breakdown into different types of variation sources: stored energy, first week energy inflows and other reasons. The proportion of each variation type is obtained by a sensitivity analysis, 
changing only one data source of the problem from one week to another (i.e. revised inflows forecast, actual stored volumes, etc.) and computing the changes in marginal cost. Although the order in which the sensitivity study is done affects the obtained proportions, the overall picture doesn't change, and it is clear that the major source of variations is the change in first week energy inflows, which is quite intriguing for a problem that has a multi year regulation capacity. It is important to mention that, as the marginal cost is a dual variable in the operation problem, a large variation of its value does not mean that the thermal dispatch has changed much. It is possible to have big changes in marginal costs even with small changes in thermal dispatch, mainly due to the need to turn on or off a plant with fuel cost very different from the previous dispatched one.

Recalling the difference between the two types of state variables previously described, it seems clear that the second type of state variables, although uncertain and with a very high short-term variability, has more weight in the decision process. In Section 4, we show a mathematical evidence to justify this behaviour, i.e., the solution variability increase is a side effect of using an autoregressive time series model embedded in SDDP. This side effect is undesirable in hydrothermal operation problem whose solutions obtained by SDDP algorithm gives not only the goals for hydro and thermal plants generation, but also the energy spot prices. It is important to mention that this model characteristic makes the solution variability increase not only due to higher variability of the inflows, but also to the inflows uncertainty. Within a dependent framework the uncertainty regarding the next stage is smaller, and this information is then taken into account by the optimization model, which bias the scenarios generated for several stages ahead. The problem is that whenever there is a expectation reversal there is a potential for a drastic change in the solutions, which may change from a wet biased decision to a dry biased one. Actually, if we consider the number of stages until the model mean reverts, it is quite likely that there is an expectation reversal. As an example, lets consider a wet inflow recent history. The decision given by the model is going to be driven by a wet future expectation, which can happen to be wrong. In this case, if a dry inflow follows, the decision will go from one wet biased solution to a dry biased solution, when the problem is solved again in the next stage, this time considering a dry recent history. In such cases, the solution variability can be even higher than the inflows variability. 


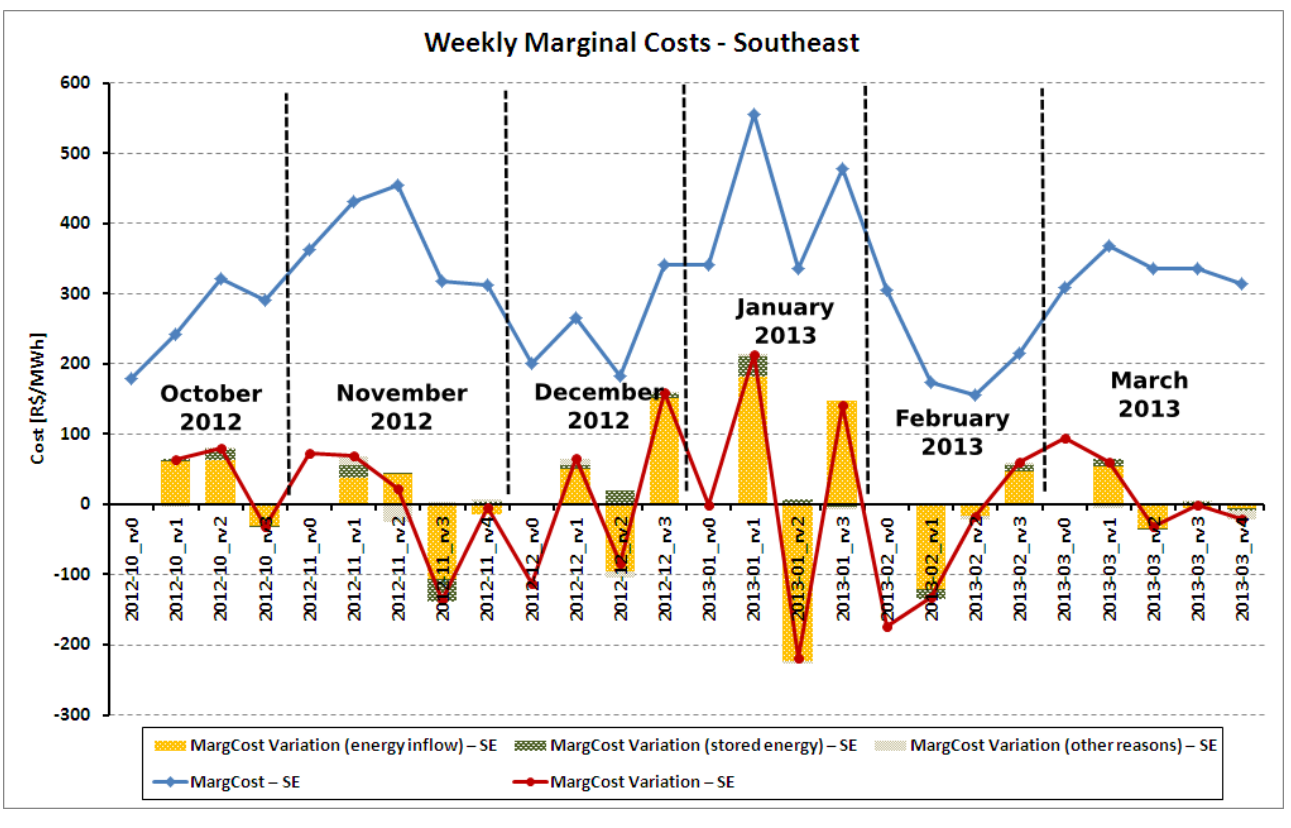

Figure 3.6: Weekly marginal costs and variation - Oct/2012-Mar/2013 


\section{4}

\section{SDDP algorithm and time dependency}

Multistage stochastic programs with recourse have become very common over the last two decades, with practical applications specially in energy problems. The success of stochastic programming, even in large scale problems, is due to the increase in computer processing capacity and the development of algorithms capable of solving them in a reasonable time. Multistage stochastic programming problems are usually solved by approximating the random variables by discrete values sampled from its probability distribution. The resulting large scale problem can be solved by optimization methods such as the Stochastic Dual Dynamic Programming (SDDP), proposed by Pereira and Pinto [3], or other sampling-based methods like Abridged Nested Decomposition (AND) [38], Convergent Cutting-Plane and Partial-Sampling Algorithm (CUPPS) [39] and ReSa [40]. These methods are extensions of Benders Decomposition to stochastic programming problems, which use sampling procedures to construct an outer approximation of the future cost function.

Sampling-based algorithms usually assume stagewise independency, which guarantees that the cost-to-go functions only depend on the current time stage, i.e., a cut calculated for a particular scenario is also valid for any other scenario at the same time stage. Even though stagewise independence is needed, the problem formulation can be modified using specific type of time dependent stochastic models for the uncertain parameters, such that stagewise independence is preserved and the SDDP method can handle it, see [3]. This is also described by [41], which shows how to consider time dependency in stochastic parameters of sampling-based algorithms. This makes possible to represent stochastic parameters using linear models, such as the autoregressive one. In some applications, such as hydrothermal operation planning problem [42], this linear uncertainty modelling can improve the solution quality to satisfactory levels, even for large scale problems, while in other applications in which more complex models are required to represent the uncertainty the linear assumption requirement is a strong limitation and hampers the SDDP usage.

Basically, the SDDP algorithm consists of two procedures, one forward in time and one backward in time. The forward procedure of the SDDP algorithm is carried out by sampling a subset of scenarios from a finite scenario tree and running a forward in time simulation for each one of the sampled scenarios. The 
most important feature of the forward procedure is to provide trial decisions for the backward step of the algorithm. Moreover, it can also be used to evaluate an upper bound for the policy value. This last feature will not be used in this work as a stopping criterion given that, as commented in [6], in large scale problems the optimality gap may be too loose to be useful. In Section 5.2.2 we are going to discuss this topic in more details.

The backward procedure of the algorithm is done backwards in time, and its objective is to estimate the cost-to-go function for each trial decision given by the forward procedure. Details of the algorithm can be found in many works, such as [3] and [14].

In some problems, with the objective of improving the solution by taking into account a more realistic representation of the uncertainties, the stochastic process is modelled by time series models, which requires proper adjustments on the state space modelling before resorting to SDDP algorithm. In the hydrothermal operation problem, modelling the time dependency of the inflows may have consequences other than an improvement on the problem representation. A question that is very often risen on Brazilian energy operation problem is why does the variability of the inflows forecasts seem to impact the decision so much more than the stored volumes for the optimization problem. Indeed, the impact observed on the (primal and dual) solution variables of stage $t$ is higher for a unit change in past inflows when compared to a unit change on the initial volume, as it was mentioned in Chapter 3. Although both variables change the right hand side (RHS) of the same equation of the problem, a change in the inflows forecast has a direct impact on the expected cost for the future stages.

In this section we are going to describe the Benders cut calculation procedure in a SDDP iteration and show that, with time dependent stochastic process, the decisions of the algorithm considers the past inflows to be more important than the stored volumes. As the inflows are more volatile than stored volumes, the results of the dependent SDDP are more volatile than the ones that uses independent time series model.

\section{1}

\section{Independent SDDP algorithm}

Assuming a discrete probability space with a stage-wise independent process $\xi_{t} \in\left\{\xi_{t}^{1}, \ldots, \xi_{t}^{N_{t}}\right\}, \forall t \in\{2, \ldots, T\}$, let us define a $T$-stage stochastic programming problem by the dynamic programming equations (4-1) and (4-2): 


$$
\begin{aligned}
\widetilde{Q}_{t}\left(x_{t-1}, \xi_{t}\right)=\min _{x_{t}} & c_{t}^{\top} \cdot x_{t}+\widetilde{\mathcal{Q}}_{t+1}\left(x_{t}\right) \\
\text { s.t. } & A_{t} x_{t}=b_{t}-B_{t} x_{t-1} \\
& x_{t} \geq 0
\end{aligned}
$$

and

$$
\begin{aligned}
z=\min _{x_{1}} & c_{1}^{\top} \cdot x_{1}+\widetilde{\mathcal{Q}}_{2}\left(x_{1}\right) \\
\text { s.t. } & A_{1} x_{1}=b_{1} \\
& x_{1} \geq 0 .
\end{aligned}
$$

In this case, the recourse function $\widetilde{Q}_{t}\left(x_{t-1}, \xi_{t}\right)$ depends on the state of the system, $x_{t-1}$, and on the uncertainty parameter $\xi_{t}=\left(b_{t}\right)$.

The main idea of the solution algorithm is to iteratively approximate the expected value of the recourse function $\widetilde{\mathcal{Q}}_{t}\left(x_{t-1}\right)$ using Benders cuts. The approximated function is denoted by $\mathfrak{Q}_{t}\left(x_{t-1}\right)$. Thereby, the approximate $t$-th stage problem after iteration $k$ of the SDDP algorithm may be written as:

$$
\begin{array}{cl}
\min _{x_{t}} & c_{t}^{\top} \cdot x_{t}+\mathfrak{Q}_{t+1}^{k}\left(x_{t}\right) \\
\text { s.t. } & A_{t} x_{t}=b_{t}-B_{t} x_{t-1} \\
& x_{t} \geq 0
\end{array}
$$

The maximum of a collection of cutting planes, $\mathfrak{Q}_{t+1}^{k}\left(x_{t}\right)$, can be expressed in a linear programming context with the use of an additional variable, $\alpha_{t+1}$. The resulting problem with $k$ cutting planes can be written as:

$$
\begin{array}{cl}
\min _{x_{t}, \alpha_{t+1}} & c_{t}^{\top} \cdot x_{t}+\alpha_{t+1} \\
\text { s.t. } & A_{t} x_{t}=b_{t}-B_{t} x_{t-1} \\
& \alpha_{t+1} \geq \widetilde{\mathcal{Q}}_{t+1}\left(x_{t, 1}^{*}\right)+\widetilde{g}_{t+1,1}^{\top} \cdot\left(x_{t}-x_{t, 1}^{*}\right) \\
& \alpha_{t+1} \geq \widetilde{\mathcal{Q}}_{t+1}\left(x_{t, 2}^{*}\right)+\widetilde{g}_{t+1,2}^{\top} \cdot\left(x_{t}-x_{t, 2}^{*}\right) \\
& \vdots \\
& \alpha_{t+1} \geq \widetilde{\mathcal{Q}}_{t+1}\left(x_{t, k}^{*}\right)+\widetilde{g}_{t+1, k}^{\top} \cdot\left(x_{t}-x_{t, k}^{*}\right) \\
& x_{t} \geq 0, \alpha_{t+1} \geq 0 .
\end{array}
$$

To calculate a $k$-th cut for stage $t-1$ using a decision point $x_{t-1, k}^{*}$, the problem (4-4) is solved for all $b_{t} \in\left\{b_{t}^{1}, \cdots, b_{t}^{N_{t}}\right\}$. Let $\widetilde{Q}_{t, j}\left(x_{t-1, k}^{*}\right)$ be the optimal value of problem (4-4) for $b_{t}^{j}$, and $\pi_{t, k, j}^{*}$ its optimal dual variable vector. The cut for stage $t-1$ is:

$$
\alpha_{t} \geq \widetilde{\mathcal{Q}}_{t}\left(x_{t-1, k}^{*}\right)+\widetilde{g}_{t, k}^{\top} \cdot\left(x_{t-1}-x_{t-1, k}^{*}\right)
$$

where $\widetilde{\mathcal{Q}}_{t}\left(x_{t-1, k}^{*}\right)=\frac{1}{N_{t}} \sum_{j=1}^{N_{t}} \widetilde{Q}_{t, j}\left(x_{t-1, k}^{*}\right)$ and $\widetilde{g}_{t, k}=-\frac{1}{N_{t}} \sum_{j=1}^{N_{t}} \pi_{t, k, j}^{*} B_{t}$. 


\subsection{1}

\section{Example: hydrothermal operation planning}

To illustrate the above procedure and to help us analyse the impact of a change in the problem's RHS, let's consider a small and simplified hydrothermal generation problem with $\mathrm{T}$ stages, one hydro plant and one thermal plant. The SAA problem defined by dynamic programming equations is given by

$$
\begin{array}{cl}
z_{1}=\min _{g t_{1}, g h_{1}, v_{1}} & c_{1} \cdot g t_{1}+\widetilde{\mathcal{Q}}_{2}\left(v_{1}\right) \\
\text { s.t. } & \\
& g t_{1}+g h_{1}=D_{1} \\
& g h_{1}+v_{1}=a_{1}+v_{0}
\end{array}
$$

and

$$
\begin{array}{cl}
\widetilde{Q}_{t}\left(v_{t-1}, \xi_{t}\right)=\min _{g t_{t}, g h_{t}, v_{t}} & c_{t} \cdot g t_{t}+\widetilde{\mathcal{Q}}_{t+1}\left(v_{t}\right) \\
\text { s.t. } & \\
& g t_{t}+g h_{t}=D_{t} \\
& g h_{t}+v_{t}=a_{t}+v_{t-1},
\end{array}
$$

where the uncertainty parameter refers only to inflow $a_{t}$ to the hydro plant, that is, $a_{t}\left(\xi_{t}\right)$, and is considered independent of $\xi_{t-1}$. The decision variables of stage $t$ are: thermal generation $\left(g t_{t}\right)$, hydro generation $\left(g h_{t}\right)$ and final stored volume $\left(v_{t}\right)$. The unit cost of thermal plant is given by $c_{t}$ and the energy demand is $D_{t}$.

In this example, our main interest is to investigate the impact of variations in the initial stored volumes $V_{0}$ and first stage inflows $a_{1}$ in the problem solution. Let's consider a three stages problem, $T=3$, and analyse the first iteration of the SDDP algorithm. For a given trial decision $v_{2}^{*}$ and considering $a_{3}^{j}$ sampled from the discretized probability distribution of the inflows, $\left\{a_{3}^{1}, \cdots, a_{3}^{N_{3}}\right\}$, the third stage problem for $a_{3}^{j}$ may be written as

$$
\begin{array}{lll}
\min _{g t_{3}, g h_{3}, v_{3}} & c_{3} \cdot g t_{3} \\
\text { s.t. } & & \\
& g t_{3}+g h_{3}=D_{3} & \left(\pi_{d, 3, j}^{*}\right) \\
& g h_{3}+v_{3}=a_{3}^{j}+v_{2}^{*} & \left(\pi_{h b, 3, j}^{*}\right)
\end{array}
$$

with optimal value $\widetilde{Q}_{3, j}\left(v_{2}^{*}\right)$, where $\pi_{d, 3, j}^{*}$ denotes the demand supply equation optimal simplex multiplier and $\pi_{h b, 3, j}^{*}$ the hydro balance equation optimal simplex multiplier.

The Benders cut for stage 2 can be calculated as:

$$
\alpha_{3} \geq \widetilde{\mathcal{Q}}_{3}\left(v_{2}^{*}\right)+\widetilde{\pi}_{h b, 3} \cdot\left(v_{2}-v_{2}^{*}\right)
$$

where $\widetilde{\pi}_{h b, 3}=\frac{1}{N_{3}} \sum_{j=1}^{N_{3}} \pi_{h b, 3, j}^{*}$ and $\widetilde{\mathcal{Q}}_{3}\left(v_{2}^{*}\right)=\frac{1}{N_{t}} \sum_{j=1}^{N_{t}} \widetilde{Q}_{3, j}\left(v_{2}^{*}\right)$. 
This cut is added to approximate the second stage problem, which can be written, for $v_{1}^{*}$ trial decision and for all $a_{2}^{j} \in\left\{a_{2}^{1}, \cdots, a_{2}^{N_{2}}\right\}$, as:

$$
\begin{array}{lll}
\min _{\substack{g t_{2}, g h_{2}, v_{2}, \alpha_{3} \\
\text { s.t. }}} & c_{2} \cdot g t_{2}+\alpha_{3} & \\
& & \\
& g t_{2}+g h_{2}=D_{2} & \left(\pi_{d, 2, j}^{*}\right) \\
& g h_{2}+v_{2}=a_{2}^{j}+v_{1}^{*} & \left(\pi_{h b, 2, j}^{*}\right) \\
& \alpha_{3} \geq \widetilde{\mathcal{Q}}_{3}\left(v_{2}^{*}\right)+\widetilde{\pi}_{h b, 3} \cdot\left(v_{2}-v_{2}^{*}\right) & \left(\pi_{c, 2, j}^{*}\right)
\end{array}
$$

where $\pi_{c, 2, j}^{*}$ refers to the Benders cut optimal dual variables. The Benders cut for stage 1 can be calculated as:

$$
\alpha_{2} \geq \widetilde{\mathcal{Q}}_{2}\left(v_{1}^{*}\right)+\widetilde{\pi}_{h b, 2} \cdot\left(v_{1}-v_{1}^{*}\right)
$$

and the first stage problem results as:

$$
z_{1}=\min _{g t_{1}, g h_{1}, v_{1}, \alpha_{2}} c_{1} \cdot g t_{1}+\alpha_{2}
$$

s.t.

$$
\begin{array}{ll}
g t_{1}+g h_{1}=D_{1} & \left(\pi_{d, 1}\right) \\
g h_{1}+v_{1}=a_{1}+v_{0} & \left(\pi_{h b, 1}\right) \\
\alpha_{2} \geq \widetilde{\mathcal{Q}}_{2}\left(v_{1}^{*}\right)+\widetilde{\pi}_{h b, 2} \cdot\left(v_{1}-v_{1}^{*}\right) & \left(\pi_{c, 1}\right) .
\end{array}
$$

From the first stage problem one can see that the impact of a variation in the initial stored volume $v_{0}$ on $z_{1}$ is equal to $\pi_{h b, 1}$, which is equal to the impact given by the same variation in hydro plant inflow $a_{1}$. This property is a consequence of the assumed time independence of the hydrological stochastic process, and is going to be illustrated by a numerical example.

\section{Numerical Example}

The characteristics of the hydrothermal problem in this example are described below. For simplicity and to avoid conversions from water inflows to generated energy, all numbers refer to the same energy unit, which was omitted. The demand at each stage is $D=50$ energy units.

\section{Hydro plant:}

- Initial stored energy $v_{0}=30$.

- First stage inflows forecast $a_{1}=18$.

- Second and third stages scenarios: $a_{t}=(4,15,25), t=2,3$.

\section{Thermal plant:}

- Thermal plant cost $c=90$ monetary units per energy unit.

- Unlimited maximum capacity. 
The sampled trial points for the current iteration were: $v_{1}^{*}=25$ and $v_{2}^{*}=30$. The third stage problem needs to be solved for each scenario considering the starting volume $v_{2}^{*}$.

1. Scenario $a_{3}^{1}=4$ :

The problem can be written as:

$$
\begin{array}{clc}
\min _{g t_{3}, g h_{3}, v_{3}} & 90 \cdot g t_{3} \\
\text { s.t. } & & \\
& g t_{3}+g h_{3}=50 & \left(\pi_{d, 3,1}^{*}\right) \\
& g h_{3}+v_{3}=4+30 \quad\left(\pi_{h b, 3,1}^{*}\right)
\end{array}
$$

The optimal solution is $g h_{3}=34, v_{3}=0, g t_{3}=16$ and $\widetilde{Q}_{3,1}\left(v_{2}^{*}\right)=$ $16 \cdot 90=1440$. The dual variables are $\pi_{d, 3,1}^{*}=90$ and $\pi_{h b, 3,1}^{*}=-90$, which means that a one unit increase in the right hand side of the problem's constraints increases the objective function by 90 , if this increase happens in the demand supply equation, and decreases the objective function by 90 if this change happens in the hydro balance equation.

2. Scenario $a_{3}^{2}=15$ :

The problem can be written as:

$$
\begin{array}{clc}
\underset{g t_{3}, g h_{3}, v_{3}}{\min } & 90 \cdot g t_{3} \\
\text { s.t. } & & \\
& g t_{3}+g h_{3}=50 & \left(\pi_{d, 3,2}^{*}\right) \\
& g h_{3}+v_{3}=15+30 & \left(\pi_{h b, 3,2}^{*}\right)
\end{array}
$$

The optimal solution is $g h_{3}=45, v_{3}=0, g t_{3}=5$ and $\widetilde{Q}_{3,2}\left(v_{2}^{*}\right)=5 \cdot 90=$ 450. The dual variables are $\pi_{d, 3,2}^{*}=90$ and $\pi_{h b, 3,2}^{*}=-90$.

3. Scenario $a_{3}^{3}=25$ :

The problem can be written as:

$$
\begin{array}{clc}
\underset{\substack{g t_{3}, g h_{3}, v_{3} \\
\text { s.t. }}}{\min } & 90 \cdot g t_{3} \\
& g t_{3}+g h_{3}=50 & \left(\pi_{d, 3,3}^{*}\right) \\
& g h_{3}+v_{3}=25+30 & \left(\pi_{h b, 3,3}^{*}\right)
\end{array}
$$

The optimal solution is $g h_{3}=50, v_{3}=5, g t_{3}=0$ and $\widetilde{Q}_{3,2}\left(v_{2}^{*}\right)=0$. The dual variables are $\pi_{d, 3,3}^{*}=0$ and $\pi_{h b, 3,3}^{*}=0$.

The average cut to be added to the second stage problem is:

$$
\alpha_{3} \geq \frac{(1440+450+0)}{3}+\frac{(-90-90+0)}{3} \cdot\left(v_{2}-30\right)
$$


which gives:

$$
\alpha_{3} \geq 2430-60 \cdot v_{2}
$$

The second stage problem considering trial point $v_{1}^{*}$ is solved for each scenario, and the cut for the first stage is calculated.

1. Scenario $a_{2}^{1}=4$ :

The problem can be written as:

$$
\begin{array}{clc}
\min _{g t_{2}, g h_{2}, v_{2}} & 90 \cdot g t_{2}+\alpha_{3} & \\
\text { s.t. } & & \\
& g t_{2}+g h_{2}=50 & \left(\pi_{d, 2,1}^{*}\right) \\
& g h_{2}+v_{2}=4+25 & \left(\pi_{h b, 2,1}^{*}\right) \\
& \alpha_{3} \geq 2430-60 \cdot v_{2} & \left(\pi_{c, 2,1}^{*}\right)
\end{array}
$$

The optimal solution is $g h_{2}=29, v_{2}=0, g t_{2}=21$ and $\widetilde{Q}_{2,1}\left(v_{1}^{*}\right)=$ $21 \cdot 90+2430=1890+2430=4320$. The dual variables are $\pi_{d, 2,1}^{*}=90$ and $\pi_{h b, 2,1}^{*}=-90$.

2. Scenario $a_{2}^{2}=15$ :

The problem can be written as:

$$
\begin{array}{clc}
\min _{g t_{2}, g h_{2}, v_{2}} & 90 \cdot g t_{2}+\alpha_{3} & \\
\text { s.t. } & & \\
& g t_{2}+g h_{2}=50 & \left(\pi_{d, 2,2}^{*}\right) \\
& g h_{2}+v_{2}=15+25 & \left(\pi_{h b, 2,2}^{*}\right) \\
& \alpha_{3} \geq 2430-60 \cdot v_{2} & \left(\pi_{c, 2,2}^{*}\right)
\end{array}
$$

The optimal solution is $g h_{2}=40, v_{2}=0, g t_{2}=10$ and $\widetilde{Q}_{2,2}\left(v_{1}^{*}\right)=$ $10 \cdot 90+2430=900+2430=3330$. The dual variables are $\pi_{d, 2,2}^{*}=90$ and $\pi_{h b, 2,2}^{*}=-90$.

3. Scenario $a_{2}^{3}=25$ :

The problem can be written as:

$$
\begin{array}{clc}
\min _{g t_{2}, g h_{2}, v_{2}} & 90 \cdot g t_{2}+\alpha_{3} & \\
\text { s.t. } & & \\
& g t_{2}+g h_{2}=50 & \left(\pi_{d, 2,3}^{*}\right) \\
& g h_{2}+v_{2}=25+25 & \left(\pi_{h b, 2,3}^{*}\right) \\
& \alpha_{3} \geq 2430-60 \cdot v_{2} & \left(\pi_{c, 2,3}^{*}\right)
\end{array}
$$

The optimal solution is $g h_{2}=50, v_{2}=0, g t_{2}=0$ and $\widetilde{Q}_{2,3}\left(v_{1}^{*}\right)=2430$. The dual variables are $\pi_{d, 2,3}^{*}=90$ and $\pi_{h b, 2,3}^{*}=0$. 
The average cut to be added to the first stage problem is:

$$
\alpha_{2} \geq \frac{(4320+3330+2430)}{3}+\frac{(-90-90+0)}{3} \cdot\left(v_{1}-25\right)
$$

which gives:

$$
\alpha_{2} \geq 4860-60 \cdot v_{1}
$$

The first stage problem can be written as:

$$
\begin{array}{clc}
\min _{g t_{1}, g h_{1}, v_{1}} & 90 \cdot g t_{1}+\alpha_{2} & \\
\text { s.t. } & & \\
& g t_{1}+g h_{1}=50 & \left(\pi_{d, 1}^{*}\right) \\
& g h_{1}+v_{1}=a_{1}+v_{0} & \left(\pi_{h b, 1}^{*}\right) \\
& \alpha_{2} \geq 4860-60 \cdot v_{1} & \left(\pi_{c, 1}^{*}\right)
\end{array}
$$

The solution of this problem with $a_{1}=18$ and $v_{0}=30$ is $g h_{1}=48$, $v_{1}=0, g t_{1}=2$ and $\widetilde{Q}_{1}\left(v_{0}\right)=2 \cdot 90+4860=5040$, with dual variables $\pi_{d, 1}^{*}=90$ and $\pi_{h b, 1}^{*}=-90$. In other words, within the independent approach, the impact of a unit variation on either $a_{1}$ and $v_{0}$ is given by $\pi_{h b, 1}^{*}=-90$, that is, both sources of information are considered equally important within the independent approach. On the other hand, as it is going to be discussed in the next section, if the stochastic process is assumed to be autoregressive, the impact of an additional resource is different depending if it comes from an increment in initial stored volume or from hydro plant inflow.

\section{2}

\section{SDDP algorithm with time dependent time series model}

An independent time series model is usually too simple to represent the stochastic process properly. An usual approach to get a more realistic representation of the underlying stochastic process is to resort to a linear model, such as a periodic autoregressive model (PAR). Unlike the independent case shown in Section 4.1, with such a dependent representation, the impacts of the variation of different components of the state vector on the solution are different. For instance, in the hydrothermal problem, it is observed that the solution becomes more sensitive to variations in the initial inflows forecast than to the initial stored volumes. This section formalizes this result.

Let's consider that $b_{t}$ is modelled as a linear function of $b_{t-1}$, that is, $b_{t}=\phi_{t} \cdot b_{t-1}+\varepsilon_{t}$, where $\phi_{t}$ is a diagonal matrix and $\varepsilon_{t}$ is a vector of constants of proper dimension. The $t$-th stage problem can then be written as: 


$$
\begin{aligned}
\widetilde{Q}_{t}\left(x_{t-1}, b_{t-1}, \xi_{t}\right)=\min _{x_{t}} & c_{t}^{\top} \cdot x_{t}+\widetilde{\mathcal{Q}}_{t+1}\left(x_{t}\right) \\
\text { s.t. } & A_{t} x_{t}=\left[\begin{array}{ll}
-B_{t} & \phi_{t}
\end{array}\right] \cdot\left[\begin{array}{c}
x_{t-1} \\
b_{t-1}
\end{array}\right]+\varepsilon_{t} \\
& x_{t} \geq 0 .
\end{aligned}
$$

Note that the probability distribution of $\xi_{t}=\left(\varepsilon_{t}\right)$ is considered to be known and independent of state variables $x_{t-1}$ and $b_{t-1}$. This problem is solved by the same approach of the independent case for $\varepsilon_{t} \in\left\{\varepsilon_{t}^{1}, \cdots, \varepsilon_{t}^{N_{t}}\right\}$. Solving the problem using the SDDP algorithm, the approximate $t$-th stage problem at iteration $k$ is:

$$
\begin{array}{ll}
\min _{x_{t}} & c_{t}^{\top} \cdot x_{t}+\mathfrak{Q}_{t+1}^{k}\left(x_{t}\right) \\
\text { s.t. } & A_{t} x_{t}=\left[\begin{array}{ll}
-B_{t} & \phi_{t}
\end{array}\right] \cdot\left[\begin{array}{c}
x_{t-1} \\
b_{t-1}
\end{array}\right]+\varepsilon_{t} \\
& x_{t} \geq 0
\end{array}
$$

and the Benders cut calculated at iteration $k$ is written as

$$
\alpha_{t} \geq \widetilde{\mathcal{Q}}_{t}\left(x_{t-1, k}^{*}\right)+\widetilde{g}_{t, k}^{\top} \cdot\left[\begin{array}{c}
x_{t-1}-x_{t-1, k}^{*} \\
b_{t-1}-b_{t-1, k}^{*}
\end{array}\right]
$$

where the sub-gradient can be estimated as $\widetilde{g}_{t, k}=-\frac{1}{N_{t}} \sum_{j=1}^{N_{t}} \pi_{t, k, j}^{*}\left[\begin{array}{ll}B_{t} & -\phi_{t}\end{array}\right]$.

\subsection{1}

\section{Example: hydrothermal operation planning with time dependent inflows}

Consider a first iteration of the same problem described in Section 4.1, with a lag-one periodic autoregressive time series model for the inflows $a_{t}=$ $\phi_{t} a_{t-1}+\varepsilon_{t}$. For a given trial decision $\left(v_{2}^{*}, a_{2}^{*}\right)$ and considering $\varepsilon_{3} \in\left\{\varepsilon_{3}^{1}, \cdots, \varepsilon_{3}^{N_{3}}\right\}$, the third stage problem for $\varepsilon_{3}^{j}$ is:

$$
\begin{array}{lll}
\underset{c t_{3}, g h_{3}, v_{3}}{\min } & c_{3} \cdot g t_{3} & \\
\text { s.t. } & & \\
& g t_{3}+g h_{3}=D_{3} & \left(\pi_{d, 3, j}^{*}\right) \\
& g h_{3}+v_{3}=\phi_{3} a_{2}^{*}+\varepsilon_{3}^{j}+v_{2}^{*} & \left(\pi_{h b, 3, j}^{*}\right)
\end{array}
$$

with optimal value $\widetilde{Q}_{3, j}\left(v_{2}^{*}, a_{2}^{*}\right)$, where $\pi_{d, 3, j}^{*}$ denotes the demand supply equation optimal simplex multiplier and $\pi_{h b, 3, j}^{*}$ the hydro balance equation optimal simplex multiplier.

The Benders cut for stage 2 can be calculated as:

$$
\alpha_{3} \geq \widetilde{\mathcal{Q}}_{3}\left(v_{2}^{*}, a_{2}^{*}\right)+\widetilde{\pi}_{h b, 3} \cdot\left(v_{2}-v_{2}^{*}\right)+\phi_{3} \cdot \widetilde{\pi}_{h b, 3} \cdot\left(a_{2}-a_{2}^{*}\right)
$$

where $\tilde{\pi}_{h b, 3}=\frac{1}{N_{3}} \sum_{j=1}^{N_{3}} \pi_{h b, 3, j}^{*}$. 
This cut is added to the second stage approximate problem, which can be written for trial decision $\left(v_{1}^{*}, a_{1}^{*}\right)$ and $\varepsilon_{2} \in\left\{\varepsilon_{2}^{1}, \cdots, \varepsilon_{2}^{N_{2}}\right\}$ as:

$$
\min _{g t_{2}, g h_{2}, v_{2}, \alpha_{3}} c_{2} \cdot g t_{2}+\alpha_{3}
$$

s.t.

$$
\begin{array}{lc}
g t_{2}+g h_{2}=D_{2} & \left(\pi_{d, 2}\right) \\
g h_{2}+v_{2}=\phi_{2} a_{1}^{*}+\varepsilon_{2}^{j}+v_{1}^{*} & \left(\pi_{h b, 2, j}^{*}\right) \\
\alpha_{3} \geq \widetilde{\mathcal{Q}}_{3}\left(v_{2}^{*}, a_{2}^{*}\right)+\widetilde{\pi}_{h b, 3} \cdot\left(v_{2}-v_{2}^{*}\right)+\phi_{3} \cdot \widetilde{\pi}_{h b, 3} \cdot\left(a_{2}-a_{2}^{*}\right) & \left(\pi_{c, 2, j}^{*}\right) .
\end{array}
$$

The Benders cut for stage 1 can be calculated as:

$$
\alpha_{2} \geq \widetilde{\mathcal{Q}}_{2}\left(v_{1}^{*}, a_{1}^{*}\right)+\tilde{\pi}_{h b, 2} \cdot\left(v_{1}-v_{1}^{*}\right)+\phi_{2} \cdot\left(\widetilde{\pi}_{h b, 2}+\phi_{3} \widetilde{\pi}_{h b, 3} \tilde{\pi}_{c, 2}\right) \cdot\left(a_{1}-a_{1}^{*}\right)
$$

and the first stage problem is going to be:

$$
\begin{aligned}
& z_{1}=\min _{g t_{1}, g h_{1}, v_{1}} c_{1} \cdot g t_{1}+\alpha_{2} \\
& \text { s.t. } \\
& \begin{array}{lc}
g t_{1}+g h_{1}=D_{1} & \left(\pi_{d, 1}\right) \\
g h_{1}+v_{1}=a_{1}+v_{0} & \left(\pi_{h b, 1}\right) \\
\alpha_{2} \geq \widetilde{\mathcal{Q}}_{2}\left(v_{1}^{*}, a_{1}^{*}\right)+\widetilde{\pi}_{h b, 2} \cdot\left(v_{1}-v_{1}^{*}\right)+ & \\
+\phi_{2} \cdot\left(\widetilde{\pi}_{h b, 2}+\phi_{3} \widetilde{\pi}_{h b, 3} \widetilde{\pi}_{c, 2}\right) \cdot\left(a_{1}-a_{1}^{*}\right) & \left(\pi_{c, 1}\right) .
\end{array}
\end{aligned}
$$

By the problem of stage one, it is easy to see that the impact on $z_{1}$ of a unit variation on the initial stored volume $v_{0}$ is given by the hydro balance equation dual variable $\pi_{h b, 1}$, while the impact of a similar change in the inflow $a_{1}$ is equal to $\left(\pi_{h b, 1}+\phi_{2} \widetilde{\pi}_{h b, 2} \pi_{c, 1}+\phi_{2} \phi_{3} \widetilde{\pi}_{h b, 3} \widetilde{\pi}_{c, 2} \pi_{c, 1}\right)$. To compare both impacts we shall check the behaviour of the dual variables. The hydro balance equation dual variable $\pi_{h b, i} \leq 0$, as an increase in the available resources will always decrease or not change the objective function value. The dual variables $\pi_{c, i}$, on the other hand, are non-negative, as an increase in the cut RHS can only make the objective function value higher (or doesn't change it), that is, $\pi_{c, i} \geq 0$. Assuming positive autoregressive coefficients $\phi_{i}$, which is usually true for real hydrothermal operation planning problems, the impact of $a_{1}$ in the objective function is greater than the impact of initial stored volume $v_{0}$.

Note that allowing autoregressive models with orders greater than one can make this difference even higher, as the state space of the problem is going to increase. As an example, consider the same problem as before, but an order two autoregressive model in the last stage: $a_{3}=\phi_{3,1} a_{2}+\phi_{3,2} a_{1}+\varepsilon_{3}$. The remaining models stay the same. The cut for the second stage is going to have an extra term to account for the new state variable in the third stage, $a_{1}^{*}$, and 
is going to be:

$$
\alpha_{3} \geq \widetilde{\mathcal{Q}}_{3}\left(v_{2}^{*}, a_{2}^{*}\right)+\widetilde{\pi}_{h b, 3} \cdot\left(v_{2}-v_{2}^{*}\right)+\phi_{3,1} \widetilde{\pi}_{h b, 3} \cdot\left(a_{2}-a_{2}^{*}\right)+\phi_{3,2} \widetilde{\pi}_{h b, 3} \cdot\left(a_{1}-a_{1}^{*}\right) .
$$

After solving the second stage problems, the cut to be added to the first stage is:

$$
\alpha_{2} \geq \widetilde{\mathcal{Q}}_{2}\left(v_{1}^{*}, a_{1}^{*}\right)+\widetilde{\pi}_{h b, 2} \cdot\left(v_{1}-v_{1}^{*}\right)+\left[\phi_{2} \cdot\left(\widetilde{\pi}_{h b, 2}+\phi_{3,1} \widetilde{\pi}_{h b, 3} \widetilde{\pi}_{c, 2}\right)+\phi_{3,2} \widetilde{\pi}_{h b, 3} \widetilde{\pi}_{c, 2}\right] \cdot\left(a_{1}-a_{1}^{*}\right) .
$$

The impact of a change in the inflows $a_{1}$ is:

$$
\left(\pi_{h b, 1}+\phi_{2} \widetilde{\pi}_{h b, 2} \pi_{c, 1}+\phi_{2} \phi_{3,1} \widetilde{\pi}_{h b, 3} \widetilde{\pi}_{c, 2} \pi_{c, 1}+\phi_{3,2} \widetilde{\pi}_{h b, 3} \pi_{c, 1}\right)
$$

which can be even higher than the previous example depending on the autoregressive parameters. A discussion of this effect for a general case with $T$ stages and autoregressive models with orders higher than one is presented on Appendix B.

\section{Numerical Example}

The characteristics of the hydrothermal problem in this example are the same as the one presented in previous section. The main difference is that the stochastic process is now modelled by an autoregressive model of the form $a_{t}=0.9 \cdot a_{t-1}+\varepsilon_{t}$. The additional information needed in this problem is:

- Inflows trial points: $a_{1}^{*}=18$ and $a_{2}^{*}=15$.

- Sampled noises for each stage: $\varepsilon_{2}=(-12.2,-1.2,8.8)$ and $\varepsilon_{3}=$ $(-9.5,1.5,11.5)$.

The third stage problem needs to be solved for each scenario considering the starting volume $v_{2}^{*}$ and inflow $a_{2}^{*}$.

1. Scenario $a_{3}^{1}=0.9 \cdot 15-9.5=4$ :

The problem can be written as:

$$
\begin{array}{clc}
\min _{g t_{3}, g h_{3}, v_{3}} & 90 \cdot g t_{3} \\
\text { s.t. } & & \\
& g t_{3}+g h_{3}=50 & \left(\pi_{d, 3,1}^{*}\right) \\
& g h_{3}+v_{3}=4+30 & \left(\pi_{h b, 3,1}^{*}\right)
\end{array}
$$

The optimal solution is the same as for the independent case, that is, $g h_{3}=34, v_{3}=0, g t_{3}=16$ and $\widetilde{Q}_{3,1}\left(v_{2}^{*}\right)=16 \cdot 90=1440$. The dual variables are $\pi_{d, 3,1}^{*}=90$ and $\pi_{h b, 3,1}^{*}=-90$. 
The problems for scenarios $a_{3}^{2}=0.9 \cdot 15+1.5=15$ and $a_{3}^{3}=0.9 \cdot 15+11.5=$ 25 are also the same as in the third stage of last section, and will be omitted.

The average cut to be added to the second stage problem is:

$$
\alpha_{3} \geq \frac{(1440+450+0)}{3}+\frac{(-90-90+0)}{3} \cdot\left(v_{2}-30\right)+0.9 \cdot \frac{(-90-90+0)}{3} \cdot\left(a_{2}-15\right)
$$

which gives:

$$
\alpha_{3} \geq 3240-60 \cdot v_{2}-54 \cdot a_{2} .
$$

The second stage problem considering trial points $v_{1}^{*}$ and $a_{1}^{*}$ is solved for each scenario, and the cut for the first stage is calculated.

1. Scenario $a_{2}^{1}=0.9 \cdot 18-12.2=4$ :

The problem can be written as:

$$
\min _{g t_{2}, g h_{2}, v_{2}} 90 \cdot g t_{2}+\alpha_{3}
$$

s.t.

$$
\begin{array}{lc}
g t_{2}+g h_{2}=50 & \left(\pi_{d, 2,1}^{*}\right) \\
g h_{2}+v_{2}=4+25 & \left(\pi_{h b, 2,1}^{*}\right) \\
\alpha_{3} \geq 3240-60 \cdot v_{2}-54 \cdot 4 & \left(\pi_{c, 2,1}^{*}\right)
\end{array}
$$

The optimal solution is $g h_{2}=29, v_{2}=0, g t_{2}=21$ and $\widetilde{Q}_{2,1}\left(v_{1}^{*}\right)=$ $21 \cdot 90+3024=4914$. The dual variables are $\pi_{d, 2,1}^{*}=90, \pi_{h b, 2,1}^{*}=-90$ and $\pi_{c, 2,1}^{*}=1$.

2. Scenario $a_{2}^{2}=0.9 \cdot 18-1.2=15$ :

The problem can be written as:

$$
\begin{array}{clc}
\min _{g t_{2}, g h_{2}, v_{2}} & 90 \cdot g t_{2}+\alpha_{3} & \\
\text { s.t. } & & \\
& g t_{2}+g h_{2}=50 & \left(\pi_{d, 2,2}^{*}\right) \\
& g h_{2}+v_{2}=15+25 & \left(\pi_{h b, 2,2}^{*}\right) \\
& \alpha_{3} \geq 3240-60 \cdot v_{2}-54 \cdot 15 & \left(\pi_{c, 2,2}^{*}\right)
\end{array}
$$

The optimal solution is $g h_{2}=40, v_{2}=0, g t_{2}=10$ and $\widetilde{Q}_{2,2}\left(v_{1}^{*}\right)=$ $10 \cdot 90+2430=3330$. The dual variables are $\pi_{d, 2,2}^{*}=90, \pi_{h b, 2,2}^{*}=-90$ and $\pi_{c, 2,2}^{*}=1$.

3. Scenario $a_{2}^{3}=0.9 \cdot 18+8.8=25$ :

The problem can be written as: 


$$
\begin{array}{lll}
\min _{g t_{2}, g h_{2}, v_{2}} & 90 \cdot g t_{2}+\alpha_{3} & \\
\text { s.t. } & & \\
& g t_{2}+g h_{2}=50 & \left(\pi_{d, 2,3}^{*}\right) \\
& g h_{2}+v_{2}=25+25 & \left(\pi_{h b, 2,3}^{*}\right) \\
& \alpha_{3} \geq 3240-60 \cdot v_{2}-54 \cdot 25 & \left(\pi_{c, 2,3}^{*}\right)
\end{array}
$$

The optimal solution is $g h_{2}=50, v_{2}=0, g t_{2}=0$ and $\widetilde{Q}_{2,3}\left(v_{1}^{*}\right)=1890$. The dual variables are $\pi_{d, 2,3}^{*}=90, \pi_{h b, 2,3}^{*}=0$ and $\pi_{c, 2,3}^{*}=1$.

The average cut to be added to the first stage problem is:

$\alpha_{2} \geq 3378+\frac{(-90-90+0)}{3} \cdot\left(v_{1}-25\right)+0.9 \cdot\left[\frac{(-90-90+0)}{3}-54\right] \cdot\left(a_{1}-18\right)$

which gives:

$$
\alpha_{2} \geq 6724.8-60 \cdot v_{1}-102.6 \cdot a_{1}
$$

The first stage problem can be written as:

$$
\begin{array}{ccc}
\min _{g t_{1}, g h_{1}, v_{1}} & 90 \cdot g t_{1}+\alpha_{2} & \\
\text { s.t. } & & \\
& g t_{1}+g h_{1}=50 & \left(\pi_{d, 1}^{*}\right) \\
& g h_{1}+v_{1}=a_{1}+v_{0} & \left(\pi_{h b, 1}^{*}\right) \\
& \alpha_{2} \geq 6724.8-60 \cdot v_{1}-102.6 \cdot a_{1} & \left(\pi_{c, 1}^{*}\right)
\end{array}
$$

The solution of this problem with $a_{1}=18$ and $v_{0}=30$ is $g h_{1}=48$, $v_{1}=0, g t_{1}=2$ and $\widetilde{Q}_{1}\left(v_{0}\right)=2 \cdot 90+6724.8=4878$, with dual variables $\pi_{d, 1}^{*}=90, \pi_{h b, 1}^{*}=-90$ and $\pi_{c, 1}^{*}=1$. The impact of a variation on $v_{0}$ is given by $\pi_{h b, 1}^{*}=-90$, while the impact of the same variation on $a_{1}$ forecast is $\pi_{h b, 1}^{*}-102.6 \cdot \pi_{c, 1}^{*}=-192.6$, that is, 2.14 greater than the impact of $v_{0}$. This effect is cumulative with the number of stages and, depending on the autoregressive parameters, can add up to more than five times. Although the first stage solution of both independent and dependent problems were the same in this small example, this is not true for all problems, otherwise it would not matter which SDDP modelling choice to use. Indeed, as the number of thermal plants with distinct costs increases, the decision in the first stage is going to change in order to reduce the expected future cost and, hence, the total expected cost.

This characteristic of autoregressive linear models explains why in real applications, although unintentionally, the impact on decision due to state variables linked to uncertain information about availability of future resources (inflows) is greater than the one provided by variables linked to certain 
information (stored volume). This explains the higher variability of the solution when inflows are modelled as a dependent time series as compared to the independent case: the solution inherits its irregular behaviour from the most influential state variable, the inflows. 


\section{5 \\ On the solution variability reduction}

In hydrothermal operation planning problem formulation, the inflows to the hydro plants (vector $b_{t}$ ) appear on the right hand side of the hydro balance equation. The vector $b_{t}$ is usually modelled as a function of past state values, such as $b_{t}=\Phi \cdot\left[b_{t-1}, \cdots, b_{t-p}\right]^{\top}+\varepsilon_{t}$, where $p$ is the linear model order, $\Phi$ is the regression coefficients matrix and $\varepsilon_{t}$ is the innovations vector.

This type of time series model is being used together with the SDDP algorithm for years aiming at better representation of the real problem. Although we cannot deny this improvement, we cannot also deny the side effect of increasing the solution variability, as shown in Section 4.2. With the purpose of reducing such variability, in this section we propose two different formulations of the problem. The first one, aimed at reducing the thermal generation variability, is focused on controlling the variation of this variable between stages, at the cost of increasing the state space. The second one was motivated by the results of Section 4.2, and is aimed at reducing the variability of both thermal generation and marginal costs. It uses the reduction of the state space of the problem to avoid fast responses of the system to small changes in the past inflows.

\section{1}

Thermal generation stabilization: risk-averse SDDP with increased state space

The first approach described in this work to reduce the SDDP solution variability is focused mainly on the control of primal variables, in particular thermal generation. This approach increases the state space of the problem in order to make possible to control variability of any primal variable. The decision to control primal variables variability resorting to an increase the hydrothermal problem state space was also taken in [43], where additional variables were added to consider the necessity of ordering in advance the liquefied natural gas that is going to be used in some thermal plants several stages later. An application on the same problem was published in [44]. In [45] the control of primal variables related to hydro plants was proposed, and [46] proposes the control of any primal variable variation, both works related to a deterministic hydrothermal operation programming problem with an up to 
two weeks horizon.

In hydrothermal operation planning problem, one important primal variable is thermal generation decision, and its value changes significantly over time due to changes in inflows. One possibility to stabilize individual thermal generation is to add, for each thermal plant, a state variable to the cost-to-go function and, in the objective function, penalize any changes greater than a threshold. The downside of this approach is that the state space of the problem can become significantly large and an unnecessary computational burden is added to the problem. Another possibility, more pragmatic, is to consider that we want to stabilize the total thermal generation in each system, and not individual generations. This is a much simpler approach, as only one state variable for each system should be added to the problem, and is also more coherent with our main objective, that is to restrain total amount of thermal generation within a certain range from one stage to another.

This proposal needs three main changes in the original problem:

1. The current stage total thermal generation needs to be calculated by a constraint and an additional variable in the problem, in this case, $g T_{t, m}$, which is the total thermal generation at stage $t$ and system $m$.

2. Constraints on thermal generation $g T_{t, m}$ must also be added. In this work, we want to restrict $g T_{t, m}$ to vary between $(1+R)$ and $(1-R)$ of the last stage thermal generation $g T_{t-1, m}$. The constant $R \in(0,1)$ is responsible for how much free variability is allowed in the problem. In order to allow changes greater than the desired range and to penalize such variations, slack variables must also be considered in such constraints.

3. The last change in the problem formulation is to consider, in the objective function, a penalty term for the variations outside the range $\left[(1-R) \cdot g T_{t-1, m},(1+R) \cdot g T_{t-1, m}\right]$

The choice of $R \in(0,1)$ was made to limit the thermal generation $g T_{t, m}$ interval choice to $\left[0,2 g T_{t-1, m}\right]$, but it is not a requirement of the proposed approach. 
The hydrothermal problem can be written, for all $t=T, \ldots, 1$ as:

$\widetilde{Q}_{t}\left(v_{t-1}, g T_{t-1}, \xi_{t}\right)=\min _{x_{t}} c t_{t} \cdot g t_{t}+\sum_{s=1}^{N S} c d \cdot d_{t, s}+p_{u p} \cdot \delta_{u p}+p_{\text {down }} \cdot \delta_{\text {down }}+\beta \cdot \mathfrak{Q}_{t+1}$

s.t.

$v_{t}+g h_{t}+s_{t}=v_{t-1}+a_{t}$

$v_{t} \leq \bar{v}_{t}$

$g h_{t} \leq \overline{g h}_{t}$

$\underline{g t}_{t} \leq g t_{t} \leq \overline{g t}_{t}$

$g h_{t, s}+\sum_{j \in N T_{s}} g t_{t, j}+n f_{t, s}+\sum_{i=1}^{N D} d_{t, s, i}=D_{t, s}, \forall s=1, \ldots, N S$

$n f_{t, s}=\sum_{l=1}^{N S}\left(f_{t, l, s}-f_{t, s, l}\right), \forall s=1, \ldots, N S$

$\left|f_{t, s, l}\right| \leq \bar{f}_{t, s, l}, \forall s=1, \ldots, N S ; \forall l=1, \ldots, N S$

$g T_{t, s}-\sum_{j \in N T_{s}} g t_{t, j}=0, \forall s=1, \ldots, N S$

$g T_{t, s}-\delta_{u p, s} \leq(1+R) \cdot g T_{t-1, s}, \forall s=1, \ldots, N S$

$g T_{t, s}+\delta_{\text {down }, s} \geq(1-R) \cdot g T_{t-1, s}, \forall s=1, \ldots, N S$

where the decisions variable vector, $x_{t}$, is defined by $x_{t}=$ $\left(g t_{t}, g h_{t}, d_{t}, v_{t}, s_{t}, f_{t}, n f_{t}, g T_{t}, \delta_{\text {up }}, \delta_{\text {down }}\right)$. The additional problem variables are:

$g T_{t, s} \quad$ total thermal generation of system $s$ at stage $t$;

$\delta_{u p} \quad$ ramp-up constraint slack variable vector;

$\delta_{\text {down }}$ ramp-down constraint slack variable vector.

The additional problem constants are:

$p_{u p} \quad$ penalty vector of excessive thermal generation increase, of size $N S$, in $\mathrm{R} \$ / \mathrm{MWa}$;

$p_{\text {down }}$ penalty vector of excessive thermal generation decrease, of size $N S$, in $\mathrm{R} \$ / \mathrm{MWa}$;

$R$ constant in $(0,1)$ that defines the allowed variability range.

Note that the $g T_{t-1, s}$ variable, in stage $t$ problem, is a constant and is part of the state of the system. The cut calculation procedure is done in the same way as before, but now considering the stages coupling matrix $B_{t}$ of the reformulated problem.

Within this approach framework there are two possibilities to control the thermal generation variability: changing the penalty term value and changing 
the allowed range size. In this work we consider a range of $5 \%$, that is, current thermal generation is allowed to be inside the range $[95 \%, 105 \%]$ of last stage thermal generation without being penalized. The penalty term, on the other hand, is a more difficult choice, as it impacts the solution behaviour and can make the main goal to be achieved or not. While a high penalty value would for sure reduce the variability, it would also increase the total operation cost, as in some critical situations an increase in thermal generation would be avoided due to the high penalty value. A low value, on the other hand, wouldn't have a big impact on the total operation cost, but could also not reduce the solution variability to an acceptable level. Several penalty values choices are discussed in Appendix C.

One point that was not considered in the experiments performed in this work is the thermal generation seasonality that is naturally present in any hydrothermal system, mostly caused by the inflows and demand seasonality. To consider this seasonality we would have to change, at each stage, the interval of allowed thermal generation change in order to make possible for the total thermal generation to follow its natural seasonality without being penalized. In this case we would have asymmetric intervals depending on the thermal generation seasonal movement. Although it is very simple to consider this asymmetric intervals in the above formulation, the choice of the intervals would require a broader study of the systems behaviour and is out of the scope of this work. Another point worth to mention is that controlling the absolute variation of thermal generation instead of the percentage variation may be more interesting in real applications, as the percentage variation may allow very small changes in low thermal generation situations but, again, would require further investigations. A final comment, this time regarding the consideration of more than one load level: with more load levels there are different options of thermal generation stabilization, such as within each load level and within all load levels. In the medium term operation planning problem, however, it seems reasonable to control only the total thermal generation variation over time.

\section{2}

\section{Thermal generation and marginal costs stabilization: risk-averse backward-independent SDDP algorithm}

This proposal is aimed at stabilizing both primal and dual variables of the problem. In hydrothermal operation planning framework, the variables of interest are thermal generation (primal) and marginal costs (dual). The main assumption behind this proposal is that the state of the problem should 
only consider variables the give certain information about future resources availability. In hydrothermal problem this implies that the coupling between stages is a function of stored volumes only and that no information about the past inflows are used to calculate the Benders cuts during the backward step of the SDDP algorithm. An immediate consequence of such approach is the reduction in the solution variability but, on the other hand, the decision quality (in terms of cost and security) is also reduced.

To deal with this drawback, we propose a twofold approach:

1. sample the trial decisions (state values) $x_{t-1}^{*}$ using a time series model that complies with the behaviour of the nature - be it linear or not so as to guarantee that the future-cost-function is estimated for realistic values; and

2. use a risk averse weight $\lambda$ so as to compensate for not taking into account the time dependency in the backward step and avoid high costs in critical situations.

Details regarding this proposal are discussed in the following sections.

\subsection{1}

\section{Backward step of SDDP algorithm}

While the forward step has only an auxiliary function for the SDDP algorithm (to sample state trial decisions and to estimate the problem bounds), the backward step is responsible for the solution of the problem. Its major objective is to estimate piecewise affine functions representing the coupling between stages. These functions are estimated for all states sampled at the forward step of the algorithm, and constitute a lower approximation of the true cost-to-go functions of the problem.

To achieve the objective of reducing the solution variability, the proposal described in this work requires that the backward step of the algorithm is solved considering only controllable variables as states, which means that an independent time series model has to be used for the stochastic inflows process in the hydrothermal planning problem. An independent time series model does not mean that we cannot use any information to generate scenarios, it means that its distribution cannot change due to different past conditions. This implies that the backward scenarios for each stage can be sampled unconditionally from the same model used in the forward step of the algorithm. Moreover, since stagewise independence is preserved, the more information that is used to estimate the distribution of the uncertainty model - such as 
future climate information and the lag zero correlation between systems - the better the solutions can be.

Although the correlation between stages are not considered in the backward step, this information is not disregarded, as the forward simulation considers a realistic time series model to estimate the states for which the cost-togo function is calculated. However, depending on the quality of models used in the forward and backward steps, this is not enough to guarantee the same improvement ${ }^{1}$ in the solution that the algorithm with time dependency and CV@R can provide, and an additional criterion is needed to guarantee the same solution quality. For instance, consider the occurrence of a sequence of low inflows, where stages with low inflows are followed by similar low inflows stages. In this case, as the autoregressive model has the ability of generating such a dry sequence, the use of an autoregressive model allows the algorithm to obtain good solutions. On the other hand, if no information such as climate forecasts is used to condition the uncertainty distribution, an independent time series model is not going to be able to generate sequences of critical inflows with the same frequency as the PAR model. Thereafter, the obtained solution is expected to be less conservative than the one obtained with the use of a PAR model and can be insecure in low inflows situations.

To deal with these critical situations, the proposed approach considers a risk measure such as the CV@R. This risk measure in particular increases the relative weight of expensive scenarios in the cut calculation and is similar to a distortion in the uncertainty probability distribution, as each scenario is going to have a different weight in the cut calculation that is proportional to its associated cost. The resulting policy is going to be much more conservative than the one considering independent time series model and a risk neutral approach, and can be as secure as the risk averse calculated considering a PAR model, as it is going to be shown later in this work.

\subsection{2}

\section{Forward step of SDDP algorithm}

The forward step of the algorithm is a direct simulation on time, from which the system's behaviour is simulated for realistic conditions and likely state values are estimated, as well as the expected cost of the policy corresponding to the entire planning horizon. In standard risk neutral approach, this expected policy value is known to be, on average, an upper bound for the problem solution. In the proposal described in this work, this property does not

\footnotetext{
${ }^{1}$ By improvement we mean greater guaranties on meeting the demand requirements which can be associated with changes in the solution such as reduction on the risks of deficit and increase in the average of stored volumes distribution.
} 
hold because of two reasons: (i) we consider the risk averse SDDP approach; and (ii) the forward step of the algorithm makes use of a time dependent time series model, while the backward step's uncertainty model is stagewise independent. This means that the estimated operation cost cannot be used as a stopping criterion for the algorithm as in traditional approaches. Note that in real sized problems this upper bound estimate is usually of no use, as it is going to be discussed.

\section{Discussion on SDDP convergence and Stopping criteria}

Although Donohue [47] claimed that the finite convergence of SDDP algorithm should follow from the finite convergence of the Nested Decomposition algorithms [48] if the scenarios used to calculate optimality cuts are resampled at each iteration, the first formal proof of almost-sure convergence for SDDP-like algorithms was derived only in [39] for the CUPPS method. Later, Linowsky and Philpott [31] have identified some crucial conditions to guarantee the convergence of sampling-based multistage stochastic decomposition methods, such as SDDP, AND, CUPPS, and ReSa, which are the independence between forward scenarios, the need to re-sample forward scenarios at each iteration and ensure that the samples used to create it are also used in cut generation. In 2008, Philpott and Guan [32] gave a simpler proof of almostsure convergence of SDDP-like algorithms based on the finiteness of the set of distinct cut coefficients. A proof of finite convergence of the SDDP algorithm was also given by Shapiro [6] using similar assumptions. Recently, Girardeau, Lecrere and Philpott [33] gave a more general proof of convergence for models with convex sub-problems, and Barty [49] proves SDDP convergence for a general convex stochastic control program, not necessarily linear, without making assumptions on the finite representation of randomness.

Despite the finite convergence of SDDP, on real problems the number of possible scenarios and cuts can be very large, and hence the time required for an algorithm to converge to the true optimum could be unreasonable [6]. Indeed, on practical applications the SDDP algorithm has to be stopped before the optimal solution is found. The standard stopping criteria for the SDDP method was proposed in [3], and consists of stopping the algorithm when the lower bound reaches the lower end of the upper bound confidence interval. This criterion can lead to premature termination when the sample size is small [50], that is, it can be too optimistic with respect to the solution optimality [6].

Hindsberger and Philpott [50] compares the SDDP standard stopping criteria to more restrictive statistical ones and to non statistical methods based on the lower bound stabilization during the iteration process. The results of 
this paper show that some of the non-statistical criteria are superior due to a better compromise between CPU time and solution quality. The authors, on the other hand, point out that the non-statistical criteria lack the quality assessment provided by statistical ones. However, as discussed in [6], it is true that stabilization of the lower bound does not mean that the solution is close to optimality, but it is also true that statistical criteria like the standard one does not ensure the quality of the obtained solution.

Another statistical approach, proposed in [6], is to use the difference between the upper end of the upper bound's confidence interval and the lower bound. This difference gives an estimate of a statistic upper bound on the optimality gap, and may be used to stop the algorithm if it is smaller than a specified accuracy. However, as shown in [51], in large problems this gap may not become small enough in a reasonable computational time. In [51] it was also tested a criterion based on the stabilization of the policy value distribution between iterations by the use of a t-test. In paper [52] a new statistical stopping criterion was proposed, as well as some modifications on the standard criterion to avoid it stopping prematurely.

Even though all discussion on SDDP stopping criteria, the best way to stop this algorithm in large scale problems stills as an open question. In the absence of a definite way to stop the algorithm, in this work we are going to use a maximum number of iterations criterion. Although it doesn't guarantee optimality of the solution, at least it makes possible to compare different experiments in a fair basis, i.e., all experiments have the same number of calculated cuts.

\section{2 .3}

\section{SDDP algorithm description}

The SDDP algorithm for the proposed approach is presented in Algorithm 1. For simplicity, in the forward step of the algorithm it is assumed an autoregressive model of order one and one forward scenario per iteration. The stopping criterion adopted was the maximum iteration number.

The algorithm requires the cut sets for each stage - usually initialized as empty sets - the boundary condition for the last stage and a maximum number of iterations. In line 1 the lower bound estimate and iteration counter are initialized. The backward scenarios for each stage are sampled in line 2 . As the scenarios considered in the backward step are stagewise independent, these scenarios will not change during the iteration process. The scenarios for the forward step of the algorithm, on the other hand, are re-sampled at each iteration using a time series model. In this case, an order one autoregressive 
model was used in line 7 . The trial decisions $x_{t}^{*}$ are calculated in line 8 and used during the backward step, which starts in line 10. Then, the backward problem is solved for all $N_{t}$ scenarios (lines 12 to 14) and the average simplex multiplier and expected cost are calculated (line 15). The CV@R is considered in line 16, where the conditional average simplex multiplier and costs are calculated. The risk averse cut is calculated in line 17 as a convex combination of the average cut with the CV@R cut and added to the cuts set of stage $t$. Then, the lower bound is updated (line 19) and the current iteration ends (line 21). 
$\overline{\text { Algorithm } 1 \text { Proposed approach to SDDP algorithm }}$

1: Initialize: $i \leftarrow 0, \underline{z}=0$ (Lower bound)

2: Sample $N_{t}$ scenarios for backward step: $\left\{\left\{b_{t}^{j}\right\}_{1 \leq j \leq N_{t}}\right\}_{2 \leq t \leq T}$

3: while $i<i_{\max }$ do

4: $\quad$ (Forward step)

5: $\quad$ Sample 1 scenario: $\left\{\varepsilon_{t}\right\}_{2 \leq t \leq T}, \varepsilon_{1}=0$

6: $\quad$ for $t=1 \rightarrow T$ do

7: $\quad b_{t}=\Phi b_{t-1}+\varepsilon_{t}$, (or any time series model)

8: $\quad x_{t}^{*} \leftarrow \arg \min _{x_{t}}\left\{\begin{array}{l}c_{t}^{\top} x_{t}+\mathfrak{Q}_{t+1}: \\ T_{t} x_{t-1}^{*}+A_{t} x_{t}=b_{t}, \\ x_{t} \geq 0\end{array}\right\}$

9: $\quad$ end for

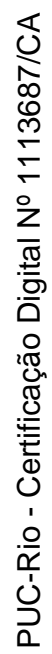

$\begin{array}{ll}\text { 10: } & \text { (Backward step) } \\ \text { 11: } & \text { for } t=T \rightarrow 2 \text { do }\end{array}$

12:

for $j=1 \rightarrow N_{t}$ do

13:

$$
\left[\widetilde{Q}_{t, j}^{*}\left(x_{t-1}^{*}\right), \pi_{t, j}^{*}\right] \leftarrow \min _{x_{t}}\left\{\begin{array}{l}
c_{t}^{\top} x_{t}+\mathfrak{Q}_{t+1}: \\
T_{t} x_{t-1}^{*}+A_{t} x_{t}=b_{t}^{j}, \\
x_{t} \geq 0
\end{array}\right\}
$$

\section{end for}

15:

$\widetilde{\mathcal{Q}}_{t}^{*}\left(x_{t-1}^{*}\right)=\frac{1}{N_{t}} \sum_{j=1}^{N_{t}} \widetilde{Q}_{t, j}^{*}\left(x_{t-1}^{*}\right) ; \widetilde{\pi}_{t}^{*}=\frac{1}{N_{t}} \sum_{j=1}^{N_{t}} \pi_{t, j}^{*}$

16 :

17:

Define $l \in\left\{1, \cdots, N_{t}\right\}$ corresponding to the $(1-\alpha)$ sample quantile of ordered $\widetilde{Q}_{t, j}^{*}\left(x_{t-1}^{*}\right)$;

$\widetilde{\mathcal{Q}}_{t}^{\prime *}\left(x_{t-1}^{*}\right)=\widetilde{Q}_{t, l}^{*}\left(x_{t-1}^{*}\right)+\frac{1}{\alpha N_{t}} \sum_{j=1}^{N_{t}}\left[\widetilde{Q}_{t, j}^{*}\left(x_{t-1}^{*}\right)-\widetilde{Q}_{t, l}^{*}\left(x_{t-1}^{*}\right)\right]_{+} ; \widetilde{\pi}_{t}^{* *}=$ $\pi_{t, l}^{*}+\frac{1}{\alpha N_{t}} \sum_{j=1}^{N_{t}} \gamma_{t, j}$

where $\gamma_{t, j}=\left\{\begin{array}{lll}0, & \text { if } & \widetilde{Q}_{t, j}^{*}\left(x_{t-1}^{*}\right)-\widetilde{Q}_{t, l}^{*}\left(x_{t-1}^{*}\right)<0 \\ \pi_{t, j}^{*}-\pi_{t, l}^{*}, & \text { if } \quad \widetilde{Q}_{t, j}^{*}\left(x_{t-1}^{*}\right)-\widetilde{Q}_{t, l}^{*}\left(x_{t-1}^{*}\right) \geq 0\end{array}\right.$

18:

$$
\text { end for }
$$$$
\mathfrak{Q}_{t}^{i}=\left\{(1-\lambda) \widetilde{\mathcal{Q}}_{t}^{*}\left(x_{t-1}^{*}\right)+\lambda \widetilde{\mathcal{Q}}_{t}^{* *}\left(x_{t-1}^{*}\right)-\left[(1-\lambda) \widetilde{\pi}_{t}^{*}+\lambda \widetilde{\pi}_{t}^{\prime *}\right]^{\top} \cdot T_{t} \cdot\left(x_{t-1}-x_{t-1}^{*}\right)\right\}
$$

$\mathfrak{Q}_{t} \leftarrow \mathfrak{Q}_{t} \cup \mathfrak{Q}_{t}^{i}$

19: $\quad$ (Lower bound update)

20: $\quad \underline{z} \leftarrow \min _{x_{1}}\left\{\begin{array}{l}c_{1}^{\top} x_{1}+\mathfrak{Q}_{2}: \\ A_{1} x_{1}=b_{1}-T_{1} x_{0}^{*}, \\ x_{1} \geq 0\end{array}\right\}$

21: $\quad i \leftarrow i+1$

22: end while 


\section{Solution Quality Indexes for Hydrothermal Operation Plan- ning Problem}

In real applications of large scale optimization problems, it is usually impossible to find an optimal solution, even with the use of parallel processing and advanced computing techniques. Moreover, we usually have several ways to model the problems in order to solve them with different techniques, each one having its own strengths and simplifications of the problem. Sometimes, when different techniques or parametrizations of the same technique are applied to a problem, it is difficult to decide whether to use one or another. This difficulty arises because of the large variety of implicit criteria involved with such problems and due to the lack of methodological ways to compare these policies.

In this work two approaches to solve the hydrothermal operation planning problem using SDDP algorithm are proposed, and we want to compare these proposals with both risk neutral and risk averse SDDP algorithms. In order to do this comparison in a way that the most important criteria are taken into account, this section presents some indexes that are later going to be used to compare different alternatives to solve the problem.

Although in hydrothermal operation planning problem the only criterion explicitly considered is cost minimization, other two criteria are important and, in a simplified way, considered as constraints or indirectly using a penalization approach in the objective function. An example is the system's security, which is considered by a penalization approach using an increasing cost for deficits, by the CV@R that penalizes the most expensive scenarios and some simplified constraints that tries to represent electrical security constraints. The other criterion, which is not represented in the problem at all, is the predictability and stability of solutions and marginal costs (and its derived curve PLD) of the problem.

In order to allow us to compare different policies with respect to these criteria, this section defines some indexes, and describes how we are going to use them to compare different approaches to solve the problem. Some of those indexes are already used in hydrothermal operation problem, while others were created to meet our purposes. Later in this work, a simplified hydrothermal operation problem is going to be used to assess the solution properties of the 
proposed and traditional approaches. The solution for each approach is going to be evaluated using the same set of 2000 scenarios, and the indexes are going to be calculated using the results for this scenarios set.

\section{1}

\section{Solution predictability and variability}

An important criterion to evaluate an hydrothermal operation planning problem policy is to assess its solution uncertainty, together with its variability. Although this is the main topic of this work, among the three criteria discussed, this is the less important one. This means that in order to achieve the main objective of this work, we cannot deteriorate the results with respect to the other two criteria.

In this section we are going to assess two types of solution variability: the predictability of the solution at each given stage for a set of scenarios and the variability over time for each scenario, during all stages of the planning horizon. Although both are going to be studied, the first type is more important, as it gives more relevant information about the uncertainty of the future solution than the second one.

\subsection{1}

\section{Solution predictability at each stage}

The first type of solution variability, and the most important one, is related to the uncertainty at each stage, and is going to be assessed in three ways, described below.

\section{Solution predictability index: 1-SAFE measure}

The first way we are going to assess the solution predictability is resorting to the concept that the volatility is directly related to the unpredictability of a variable. Unlike the unconditional uncertainty shown in previous section, the volatility of a variable at stage $t$ measures the unpredictability of stage $t$ given the past values. The proposed index is going to be called 1-SAFE, which stands for the volatility of the "one step ahead forecast error". This index can be calculated by resorting to a linear regression of type $x_{t}=\beta_{0, t}+\beta_{1, t} x_{t-1}+e_{t}$, where $x_{t}$ can be either the thermal generation or the PLD, $\beta_{1, t}$ is the regression coefficient, $\beta_{0, t}$ its intercept and $e_{t}$ the error term.

The procedure to estimate a volatility index is summarized below:

1. Run a simulation with $N$ scenarios, from stage 1 to $T$.

2. For each stage $t=2, \cdots, T$ proceed as follows: 
(a) Take all PLDs or thermal generations at stage $t-1$ and $t$ as $x_{t-1}$ and $x_{t}$.

(b) Discard all pairs $\left(x_{t-1}, x_{t}\right)$ in which there is spillage in stage $t-1$ or $t$.

(c) Fit a linear model $x_{t}=\beta_{0, t}+\beta_{1, t} x_{t-1}+e_{t}$ to the data.

(d) Calculate the models residuals by taking $\tilde{e}_{t}=x_{t}-\beta_{0, t}+\beta_{1, t} x_{t-1}$ for all scenarios.

(e) The volatility index of stage $t$ is going to be the standard deviation of the model errors, which is directly related to the predictability of the data:

$$
V I_{t}[R \$ / M W h]=s d\left(e_{t}\right) .
$$

The described procedure is going to be done for PLDs and thermal generation discarding the scenarios in which the system is spilling. The changes from spilling to not spilling and the other way round can be seen as changes in regimes, and a single linear model would not fit well both cases. Although this model is simple enough to be able to forecast situations which system is about to spill, in real world applications it is possible to forecast spillages, as information regarding reservoirs levels, together with inflows and weather forecast, is available.

The effect of discarding such values is shown for a hypothetical case study in Figure 6.1. The regression considering all points, including the red triangle ones, is shown by the dashed red line, while the regression discarding such points is shown by the blue line. As the discarded points are usually related to large forecast errors, the 1-SAFE index can be highly impacted by the increased presence of spilling in risk aversion cases. In this example, the VI index considering all the data points equals to 48.37 , while without considering the spillage points the VI index equals to 19.49. This emphasizes the importance of discarding the points in which there is spillage, as the impact on the results are huge, but could be easily predicted in most cases.

There are several other ways to compute a predictability index such as the one proposed in this work. Models considering not only past PLDs, but also the storage levels and energy inflows could give better results to forecast future PLDs and, therefore, would give lower predictability indexes. On the other hand, the relation between the results calculated for each solution approach would not change much. In the development of this variability index both storage levels and energy inflows were considered in the initial assessments, but due to the small improvement achieved and the higher complexity of the model, a simple order one regression was applied to the data. 


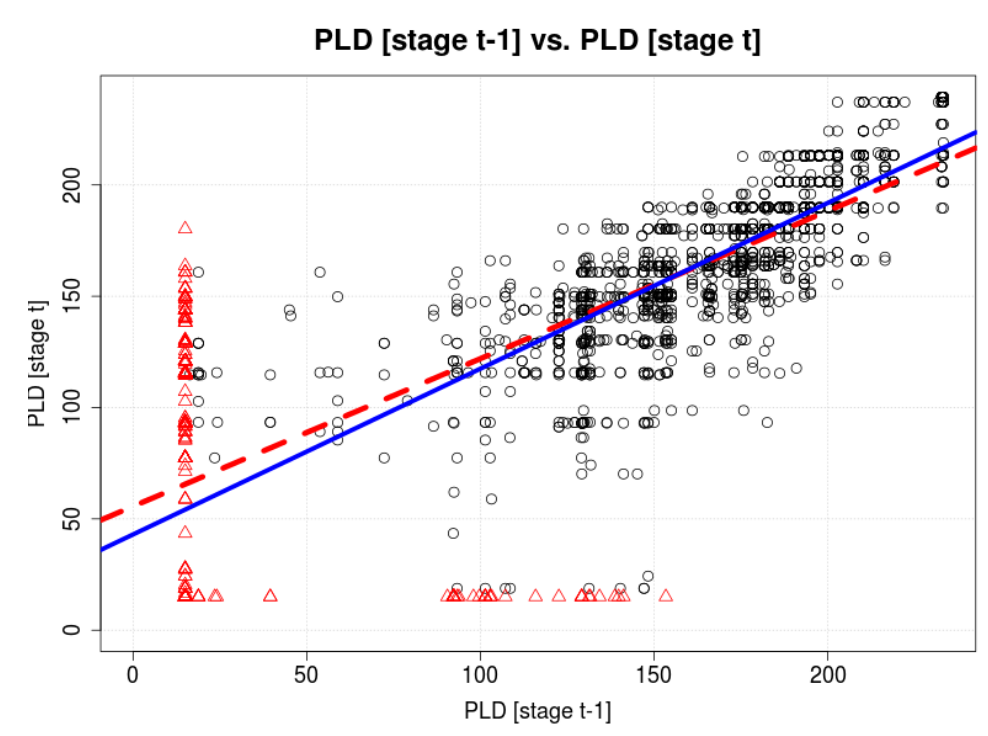

Figure 6.1: 1-SAFE index estimation - dashed red line is the regression including the red triangle spilling points; the blue line is the adopted regression, discarding such points

\section{Solution uncertainty at each stage}

The second way we are going to assess the solution variability for a given stage is by examining the graph of the average thermal generation, PLD and energy stored levels for each stage, together with their 5\% and 95\% quantiles, similarly as shown in Figure 6.2 for hypothetical energy stored levels. These results allow us to visually compare the dispersion of each distribution, together with its expected value, and is directly related to the unconditional solution uncertainty at a given stage $t$, which is important in medium-term analysis to assess the behaviour of this system index not driven by the current situation.

This result can be summarized by an index that express the overall dispersion of the variable along time. In this work we propose the use of the average $90 \%$ empirical confidence interval given by:

$$
\frac{1}{T} \sum_{t=1}^{T}\left(Q_{t}^{95 \%}-Q_{t}^{5 \%}\right),
$$

where $Q_{t}^{x \%}$ represents the $x \%$ quantile of a given variable at stage $t$.

\section{Solution variability given the same initial conditions}

In this analysis, we are going to compare the thermal generations and PLDs of the second stage of the problem. As the first stage of the problem is deterministic, the second stage problems have similar initial conditions, and differ from each other by the energy inflows to equivalent reservoirs, which are different for every sampled scenario. This implies that the distribution of 


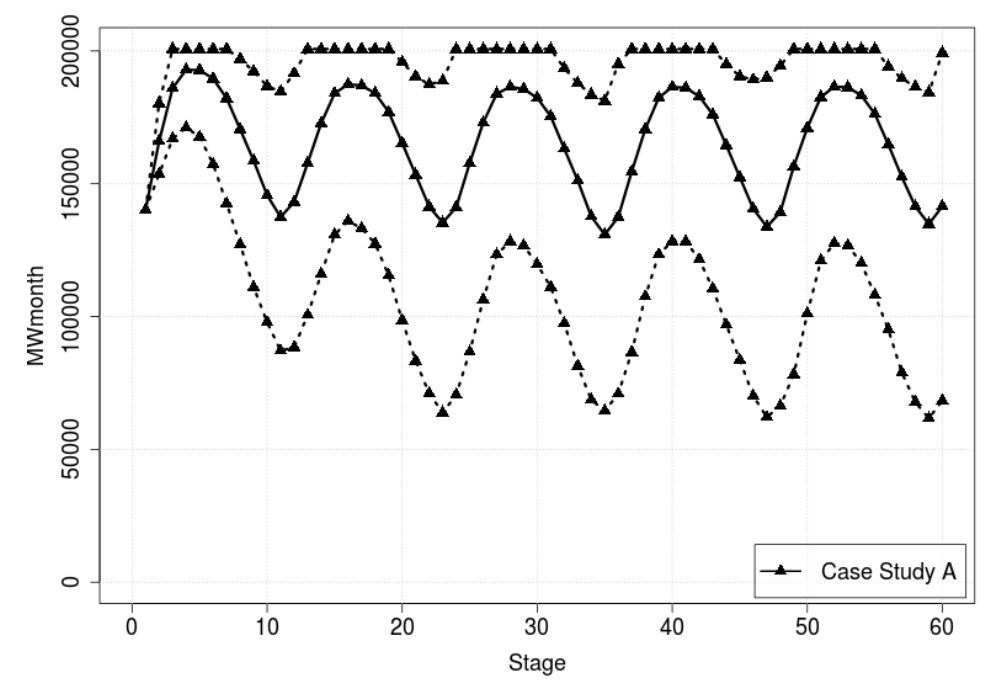

Figure 6.2: Hypothetical energy stored levels average, 5\% and 95\% quantiles for each stage [MWmonth]

the second stage solution gives us information regarding the variability of the solution with respect to changes in the inflows, which is exactly the problem that motivated this work in the first place.

These results are going to be summarized in graphs like the one shown in Figure 6.3, where we can see the second stage PLD histograms of two example case studies, together with their first stage PLDs (dashed vertical lines). It is expected that the second stage values are distributed around the first stage one, and the smaller the deviation, the better the policy may be considered.

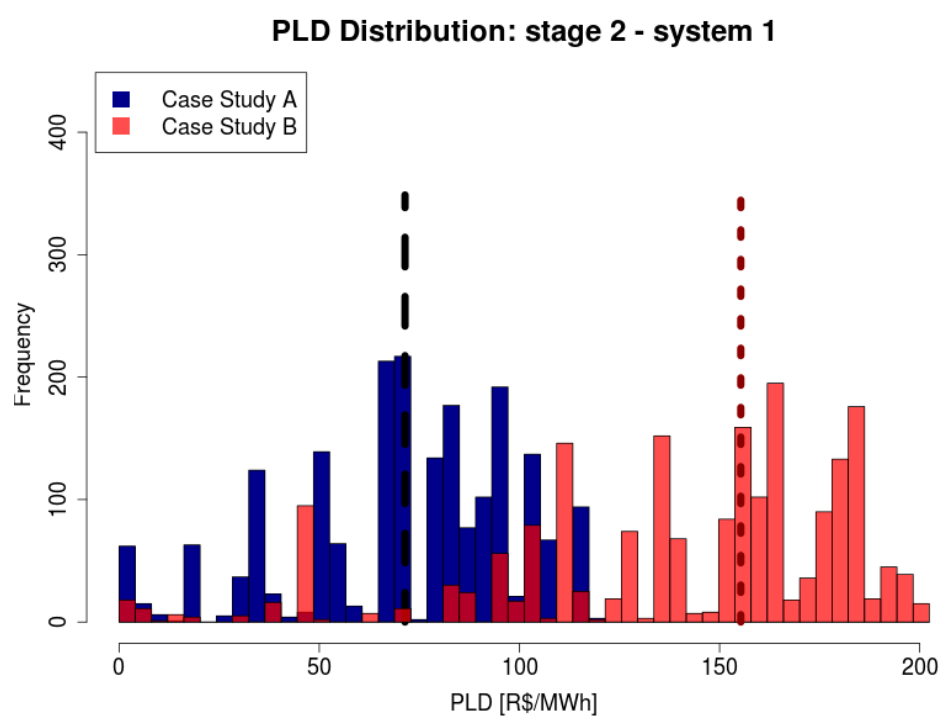

Figure 6.3: Second stage solution PLD 


\section{1 .2}

\section{Solution variability over time}

This type of solution variability is related to the variability of thermal generation over time. Figure 6.4 shows an example of a variable behaviour over time for a given scenario, in this case, the historical inflows between the years of 1936-1940. The main objective of this section is to propose a way that allows a comparison of several case studies such that it is possible to distinguish which one has the smoother variable behaviour for a set of scenarios. In this example, the Case Study B has a smoother behaviour, and the proposed index should reflect that.

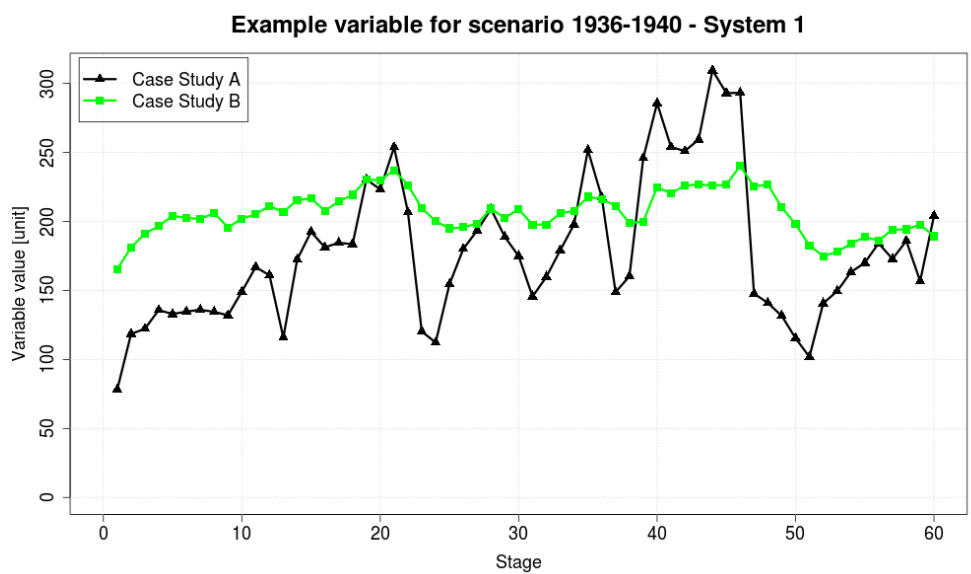

Figure 6.4: Variable results for a given scenario

For each simulated scenario $s$, we propose the following procedure to estimate a variability index:

1. Thermal generation variation over time [\%] for scenario $s$ :

$$
\delta_{s, t}[\%]=100 \times \frac{g t_{t}^{s}-g t_{t-1}^{s}}{g t_{t-1}^{s}}, \forall t=2, \cdots, T
$$

where $T$ is the total number of stages and $g t_{t}^{s}$ is the thermal generation on stage $t$ and scenario $s$.

2. For all $\delta_{s, i}$ where the marginal cost is below the minimum spot price defined for Brazilian energy market its value is discarded and considered as "missing value". This procedure is done in order to avoid contamination of the variability index due to periods when spillage is responsible for drastic drops in thermal generations and marginal costs. As spillage increases when risk averse measures are implemented, we could misleadingly correlate the use of a risk measure with an increase in solution variability. 
3. The variability of scenario $s$ is defined as:

$$
V_{s}[\%]=\frac{1}{T-1} \sum_{t=2}^{T}\left|\delta_{s, t}\right| .
$$

For each scenario we know the variability index $V_{s}$, and we can plot the distribution of this index to compare the variability of thermal generation of several approaches. The same analysis can be done to compute the variability index of PLDs. Figure 6.5 shows the distributions of an example variable, and it is clear that Case Study B has a more stable behaviour.

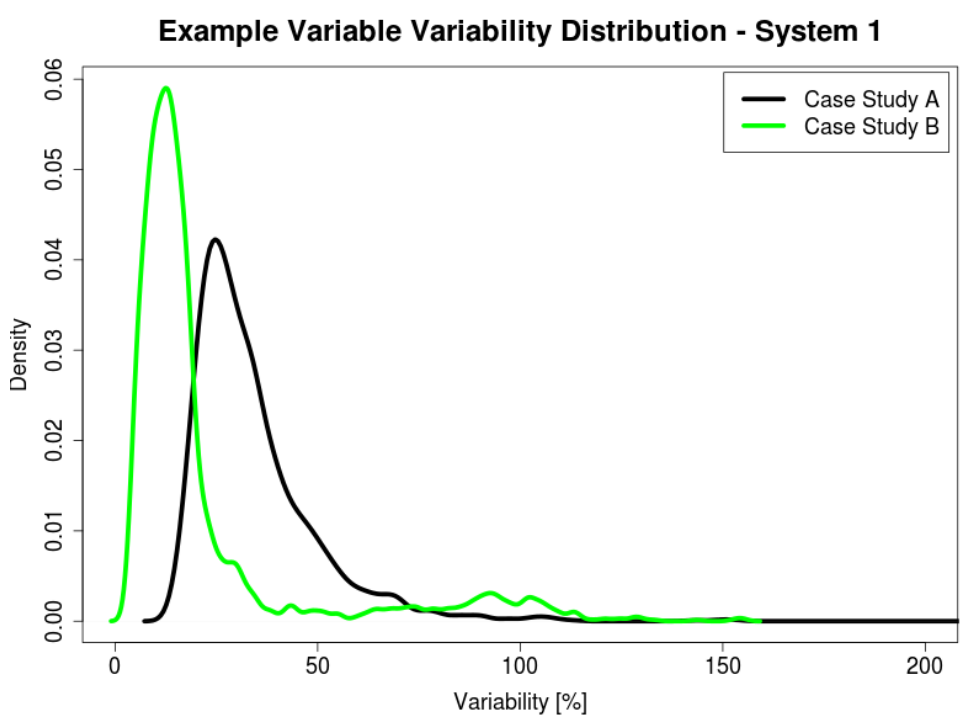

Figure 6.5: Variability index

It is important to note that the proposed index measures the average changes over time of a given scenario, and has no relation with the solution uncertainty, that is, with the easiness to forecast future values. A time series with a deterministic seasonal behaviour or a deterministic trend may show a high variability index value, but could be easily forecast. The main point in assessing the smoothness of a given variable is that a very erratic behaviour usually means that there is an extra effort to accomplish the same task that a smooth behaviour would accomplish. Although this is not always true, for thermal generation variable, for example, a stable behaviour can give the same amount of energy in a certain period of time that a solution with an erratic behaviour, and possibly with a lower cost in cases in which the obtained solution is not optimal, such as in the operation planning problem, which is too large to be solved to optimality. 


\section{2}

\section{Cost-based indexes}

The next criterion to be discussed, and for many people the most important one, is operation cost. As Brazilian laws for the energy sector are mainly guided by a low tariff policy, this criterion is the most important one, together with the security of supply. In this work we are going to present three cost-based results.

\subsection{1}

\section{Simulation costs over time}

The first one presents information about the distribution of operation costs along stages (the penalty costs at each stage are disregarded). It shows in one graph, for each stage, the average cost value and the $99 \%$ quantile of the cost distribution. With this result we can check whether one approach implies in a cost expectation increase, and for which stages this happens, and if there is a reduction on price peaks, which means that the high costs of dry scenarios were avoided. An example of this result is shown in Figure 6.6.

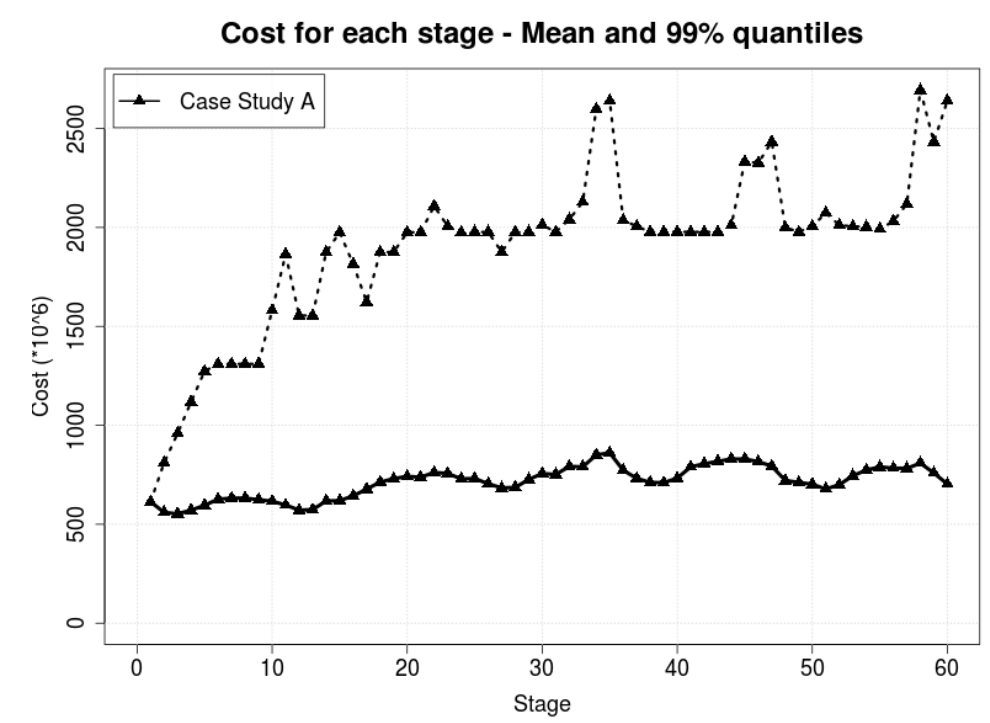

Figure 6.6: Average and 99\% quantile costs for each stage

\subsection{2}

\section{Policy values and its confidence interval}

The second result summarizes the cost information in one present value and its $95 \%$ confidence interval. For each scenario $i$, we compute the sum of the present values of all stages, such as in Equation 6-4, with an annual discount rate $r$ and a cost $c_{t, i}$ at each stage $t$. 


$$
Z_{i}=\sum_{t=1}^{T}\left(\frac{1}{\sqrt[12]{1+\frac{r}{100}}}\right)^{t-1} \cdot c_{t, i}
$$

The expectation is taken over $N$ scenarios with equal probability, as in Equation 6-5, and the expected policy value $95 \%$ confidence interval is computed as in Equation 6-6, where $s d(\cdot)$ denotes standard deviation.

$$
\begin{gathered}
\mathbb{E}[Z]=\frac{1}{N} \cdot \sum_{i=1}^{N} Z_{i} \\
C I_{\mathbb{E}[Z]}=\left[\mathbb{E}[Z]-\frac{1.96 \cdot s d(Z)}{\sqrt{N}}, \mathbb{E}[Z]+\frac{1.96 \cdot s d(Z)}{\sqrt{N}}\right]
\end{gathered}
$$

This index gives information about the uncertainty on the expected total cost estimator, and allows us to measure the expected increase in the policy value between risk neutral and the other approaches. We expect that we are able to find parametrizations of the proposed approaches that gives similar results, although we would allow a small increase in cost expectation if the solution variability is reduced. An example of such result is shown for two case studies in Figure 6.7.

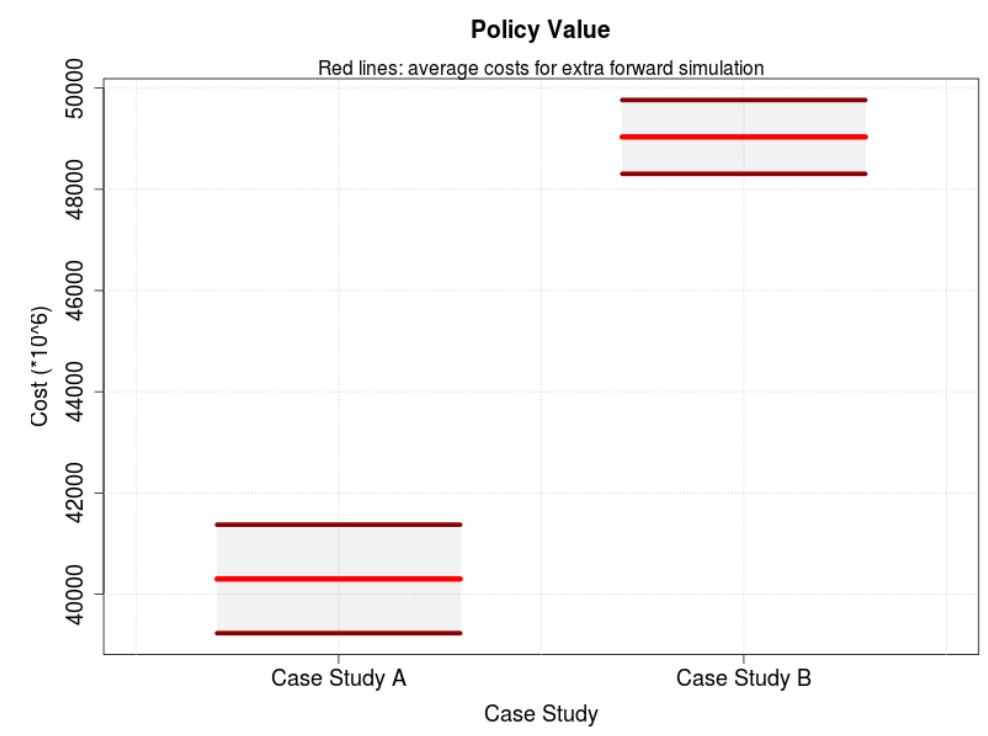

Figure 6.7: Policy values $\left[\mathrm{R} \$ \times 10^{6}\right]$ and its $95 \%$ confidence interval

\subsection{3}

\section{Conditional value at risk}

The third result reflects the average cost in the worst scenarios, and is given by the $C V @ R_{\alpha}$ risk measure:

$$
C V @ R_{\alpha}(Z)=V @ R_{\alpha}(Z)+\alpha^{-1} \mathbb{E}\left[\left(Z-V @ R_{\alpha}(Z)\right)_{+}\right]
$$


where $V @ R_{\alpha}(Z)$ is the $(1-\alpha)$-quantile of the distribution of $\mathrm{Z}$, and $(x)_{+}$is the maximum between 0 and $x$. In this work we are going to use $\alpha=0.05$, as this is an usual value in risk applications. Note that $\mathrm{Z}$ is the present value of the policy values. This index should be smaller for risk aversion cases, as the objective function considers $C V @ R$ minimization explicitly, although in the optimization the CV@R is considered in a nested formulation, and not taken over the present values of the policy values. This is an important measure, as it is directly related to the cost of worst scenarios and shows which approach deals better with dry situations.

\section{3}

\section{Continuity of energy supply}

The last criterion to be assessed is the continuity of energy supply. This criterion is often referred to as system operation security. A solution approach that disregard this criterion could have drastic economical impacts in case the continuity of energy supply is compromised. Therefore, this criterion rivals with the minimum cost objective and for some policy makers it can be considered as the most important one. The security provided by each approach is going to be assessed by two indexes: the stored energy and the annual risk of deficit.

\subsection{1}

\section{Stored Energy}

The stored hydro energy is directly related to the security of hydrothermal systems that strongly relies on hydro plants to supply its demand, such as the Brazilian one. In those cases, the more water stored in hydro plants, the longer dry period the system can manage to supply its demand without resorting to an energy rationing. The stored energy for all case studies are going to be compared in two ways.

The first way consists of a graph that shows, for each stage, the average stored energy value and the 5\% and 95\% quantiles of the stored energy distribution, as shown in Figure 6.2. This graph allows the comparison of the level and variability of the distribution. For instance, it emphasizes the effect of including risk aversion concerns in the problem: higher stored levels in the reservoirs. This result also gives a clue about the behaviour of the tails of stored volumes distribution, such as which approach keeps the reservoirs in higher levels in dry situations, for example.

Although this result gives a general overview, it lacks a more objective way of comparing the results and, in some cases, can be insufficient to distinguish between two different solutions. To deal with this lack of objectivity, 
we have created an index that results from the comparison of the stored volumes distribution at each stage. It is summarized, for each stage, as follows:

1. Calculate de empirical cumulative distribution function of the stored energy volumes for all case studies, as shown in Figure 6.8. This gives the sorted vectors $S t o V o l i$, for all case study $i$, with dimension $N$, where $N$ is the number of scenarios (in our case, 2000).

2. For all $N$ probability levels $p=\frac{1}{N}, \cdots, 1$, calculate the maximum stored volume among the case studies, which gives the vector $\overline{S t o V o l}$. This is the best solution found at each probability level, that is, it dominates all solutions, and is shown by the blue dashed line in Figure 6.8. This procedure is repeated to calculate the minimum stored volumes, StoVol.

3. For each probability level and case study $i$ calculate a score vector by dividing (term by term) its stored volume vector by the maximum values vector: $I_{i}^{t m p}=\frac{S t o V o l_{i}}{S t o V o l}$. The resulting vector $I_{i}^{t m p}$ indicates, for all probability levels, the relation of case study $i$ with the best value found, with 1 being the best possible score.

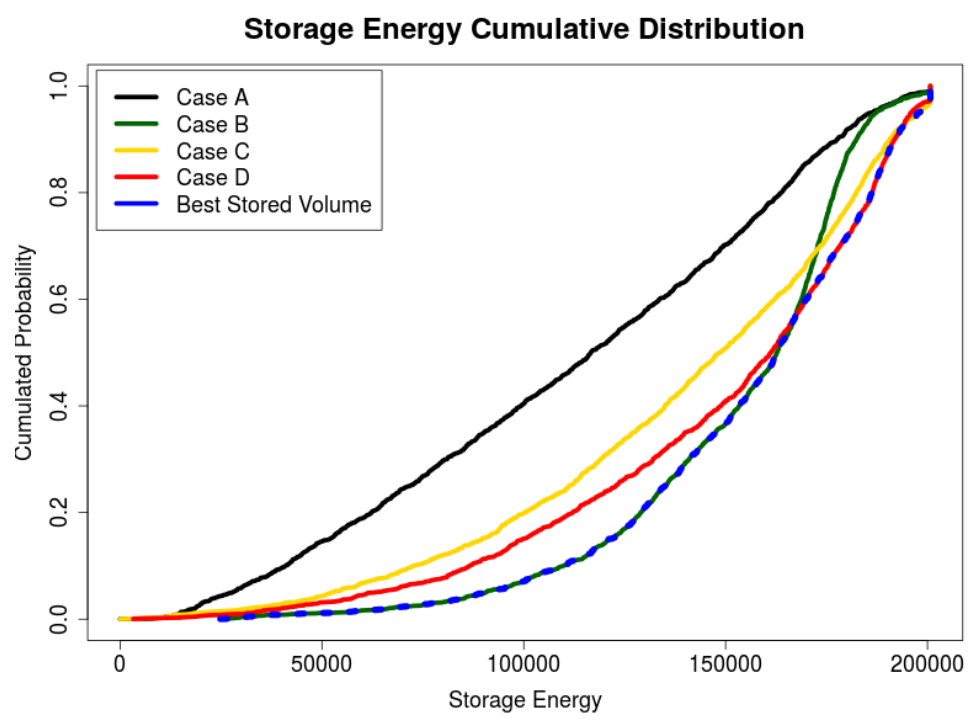

Figure 6.8: Stored Energy Volume Analysis

Although this score vector $I_{i}^{t m p}$ allows us to compare all solutions $i$ at each stage and probability level, it does not make any distinction between stored levels, that is, the importance of all scores in the vector $I_{i}^{t m p}$ is the same, no matter to which stored level it is related. From a practical point of view, it seems much more important to have the highest stored volume in low storage (dry) situations than when the reservoirs are almost at full level. To 
represent this, we define a function $f(x)$, which gives a weight for each storage level interval:

$$
f(x)= \begin{cases}1.2, & \text { if } x \in[0,30 \%] \\ 1.1, & \text { if } x \in(30 \%, 50 \%] \\ 1.0, & \text { if } x \in(50 \%, 70 \%] \\ 0.9, & \text { if } x \in(70 \%, 90 \%] \\ 0.8, & \text { if } x \in(90 \%, 100 \%] .\end{cases}
$$

The values that define this function should account for the decision maker preferences, and were chosen in a way that reflects a preference for higher stored volumes in critical situations. This function $f(x)$ is then applied to all probability levels $p$, using the minimum stored volume at each level, $\underline{S t o V o l}$, as the variable $x$ of function $f$. The resulting weights are stored in a vector $W$, which gives the importance of the scores $I_{i}^{t m p}$ for each probability level. The final index vector is calculated by an element-wise product $I_{i}=W \cdot I_{i}^{t m p}$. The quality index for case study $i$ at a given stage is $Q I_{i}=\frac{1}{N} \sum_{j=1}^{N} I_{i, j}$, where $I_{i, j}$ denotes the $j$-th element of vector $I_{i}$.

The above result allows us to compare, in one graph, the stored energy quality index in all stages for every approach. Moreover, it allows us to estimate the average quality index for each solution being compared.

\subsection{2 \\ Risks of deficit}

The last index measures security explicitly: the annual frequency of deficits for several deficit depths. For each system and deficit depth (greater than $1 \%, 5 \%, 10 \%$ and $20 \%$ of the demand), we compute how many scenarios lies within the level of deficit in each year of the study horizon, and divide by the total number of scenarios. As a result, for each case study and deficit depth, we have an annual deficit frequency. This result is shown for a hypothetical case study in Figure 6.9, where each line represents one deficit depth and the dots represent the years, from year one up to five and can be used to explicit which proposal is related to smaller risks of deficit.

\section{4}

\section{Summary indexes}

The previous topics presented a variety of ways to compare different solutions for many criteria. In order to give a brief overview of each solution approach with respect to all three criteria at a glance, this section presents a summary index for each criterion. The cost-based criterion is going to be summarized by the expected total cost and confidence interval (Section 6.2.2), which gives an idea of costs level for each approach. The second criterion, 


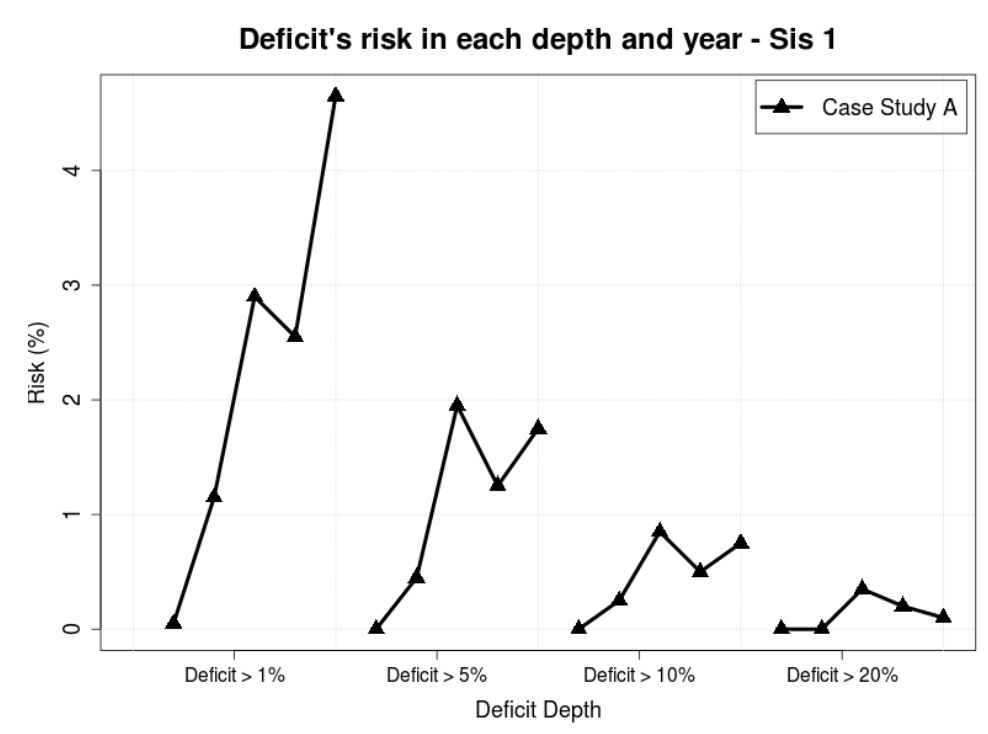

Figure 6.9: Annual risks of deficit [\%]

system security, is going to be summarized by the average of the annual deficits going to be summarized by the average $90 \%$ PLD empirical confidence interval calculated by Equation (6-2), that is, the average of the distances between 5\% and $95 \%$ quantiles of the PLD distribution. These summary indexes are not meant to decide between approaches, as they lack many details assessed in previous topics, but just to give an idea of which are the strengths of each methodology. 


\section{Computational experiments}

In order to compare both proposed approaches with standard risk neutral and risk averse SDDP algorithm, a computer implementation was written in $\mathrm{C}++$ using Soplex Solver [53], and considered the risk neutral and risk averse SDDP approaches. Detailed description of the risk neutral and risk averse algorithm can be found in [14]. The codes were run on 1 core of a quad-core Intel Xeon $3.4 \mathrm{GHz}$ with $16 \mathrm{~GB}$ RAM machine.

\section{1}

\section{Case studies description}

\subsection{1}

\section{Problem description}

The cases studies considered a hydrothermal operation planning problem and were based on a simplified Brazilian Interconnected Power System configuration, as of January 2012. The problem was discretized monthly in 120 stages (60 stages of operation planning horizon and 60 stages as a boundary condition). The system was represented as in Figure 3.2, with four energy systems interconnected by transmission lines. The PLDs were calculated as the marginal cost values, limited to the interval [12.20,727.52], which was the PLD interval used in 2012. The same yearly discount rate used in the official problem was adopted, that is, $12 \%$. The equivalent systems maximum generation values, storage availability and initial conditions are shown in Table 7.1. A thermal plants summary for each system is shown in Table 7.2, where it is possible to see the installed capacity and the price range for each system. It is important to note in both Tables 7.1 and 7.2 that the South-East system is the biggest one, and it is going to be the main focus of our analysis.

\begin{tabular}{c|c|c|c} 
System & $\begin{array}{c}\text { Maximum } \\
\text { Generation }\end{array}$ & $\begin{array}{c}\text { Maximum } \\
\text { Storage Volume }\end{array}$ & $\begin{array}{c}\text { Initial } \\
\text { Stored Volume }\end{array}$ \\
\hline South-East & 45829.1 & 200717.6 & 119428.8 \\
South & 13381.8 & 19617.2 & 11535.1 \\
North-East & 9780.9 & 51806.1 & 29548.2 \\
North & 7740.2 & 12679.9 & 6649.4
\end{tabular}

Table 7.1: Equivalent reservoirs data [MWmonth] 


\begin{tabular}{c|c|c|c} 
System & $\begin{array}{c}\text { Installed } \\
\text { Capacity [MW] }\end{array}$ & $\begin{array}{c}\text { Minimum } \\
\text { Price }[\mathrm{R} \$ / \mathrm{MWh}]\end{array}$ & $\begin{array}{c}\text { Maximum } \\
\text { Price }[\mathrm{R} \$ / \mathrm{MWh}]\end{array}$ \\
\hline South-East & 13772 & 0.01 & 1047.38 \\
South & 3630 & 53.82 & 780 \\
North-East & 4336 & 70.16 & 926.43 \\
North & 332 & 463.5 & 463.5
\end{tabular}

Table 7.2: Thermal plants summary

The demand for each system is considered deterministic, such as in Brazilian real operation planning applications. The energy inflows in equivalent systems reservoirs, on the other hand, are uncertain and are represented by the use of scenarios, modelled by a periodic autoregressive, or by an independent time series model, depending on the case study.

The load of each area must be supplied by local hydro and thermal plants or by power flows among the interconnected areas. The load shortage at each area is taken into account by a slack thermal generator with costs that increase with the amount of load curtailment (Table 7.3). The interconnection limits between areas may differ depending of the flow direction, see Table 7.4. The energy balance equation for each sub-system must be satisfied at each stage and scenario. The case's general data, such as hydro and thermal plants data and interconnections capacities were taken as static values through time, while the demand for each system and the energy inflows in each reservoir were taken as time varying.

\begin{tabular}{c|c|c} 
& $\begin{array}{c}\% \text { of total } \\
\text { load curtailment }\end{array}$ & $\begin{array}{c}\text { Cost } \\
{[\mathrm{R} \$ / \mathrm{MWh}]}\end{array}$ \\
\hline 1 & $0-5$ & 1206.38 \\
2 & $5-10$ & 2602.56 \\
3 & $10-20$ & 5439.12 \\
4 & $20-100$ & 6180.26
\end{tabular}

Table 7.3: Deficit costs and depths

It is important to emphasize again that the modelling used in this work is simplified in comparison with the official model used in Brazilian operation

\begin{tabular}{|c|c|c|c|c|c|c|}
\hline & \multicolumn{5}{|c|}{ to } \\
\hline & & $\mathrm{SE}$ & $\mathrm{S}$ & $\mathrm{NE}$ & $\mathrm{N}$ & IM \\
\hline \multirow{5}{*}{ from } & $\mathrm{SE}$ & - & 7700 & 1000 & 0 & 4000 \\
\hline & S & 5670 & - & 0 & 0 & 0 \\
\hline & $\mathrm{NE}$ & 600 & 0 & - & 0 & 3000 \\
\hline & $\mathrm{N}$ & 0 & 0 & 0 & - & - \\
\hline & IM & 2854 & 0 & 3960 & 3149 & - \\
\hline
\end{tabular}

Table 7.4: Interconnection limits between systems [MWavg] 
planning. We used the same modelling described in [14], which is able to preserve the main characteristics of the problem and its large scale size. The main simplifications were the lack of a run-of-river equivalent reservoirs, the absence of minimum flows constraints (that are responsible for a minimum generation, as each reservoir must let an amount of water to go down the river), the consideration of only one load depth, no correction to the equivalent reservoirs production due to storage levels and no water evaporation considered in the equivalent reservoirs. Although these simplifications could be seen as a weakness of the current experiments, the consideration of these problem characteristics would not reduce the solution variability and, therefore, their absence does not invalidate the results show in this section.

\subsection{2}

\section{Solution approaches description}

In order to evaluate the performance of the proposed approaches for solving multi-stage linear stochastic long-term operation planning problems, we carried out numerical experiments considering two reference cases:

- Risk neutral case: the main objective of this study is to establish a reference for the problems solution when no risk measure is taken into account. A periodic autoregressive model was used to sample the scenarios for the backward and forward procedure. This case study, a risk neutral with backward dependent procedure, is going to be called "RN BW-D" from here onwards.

- Risk averse case $(\alpha=0.05, \lambda=0.15)$ : this case study establishes a reference for a good solution in the sense that the risks of load curtailment are small and the increase in the operation cost is acceptable. It uses the same scenarios tree as the "RN BW-D" case, and is going to be called "RA BW-D ( $\alpha=0.05, \lambda=0.15)$ ". The choice of the quantile $\alpha$ was the standard used in most risk aversion applications.

Additionally, each proposal described in this work is represented by one case study. The choice of each parametrization was done empirically, and is described in Appendix C. The proposed case studies, to be compared with the reference cases, are:

- RA BW-D-GT $\left(\alpha=0.05, \lambda=0.15, \Delta_{G T}=5 \%, p=100\right)$ : chosen parametrization of thermal generation as state variable approach, with a maximum variability in the generation of thermal plants of $5 \%$ over stages, and penalty term values $p=100$. 
- $\mathrm{RA}^{+}$BW-I $(\alpha=0.05, \lambda=0.40)$ : risk averse backward independent approach, with the same CV@R level as the reference risk averse case, but with a higher risk aversion weight. This higher risk aversion is emphasized by the "+" superscript, and can be interpreted as an uncertainty (or ambiguity) aversion, and is trying to compensate the fact that we are using a distribution that is known to be different from reality.

Where clear from the context, the case studies names are going to appear without the parameter values to avoid either being repetitive or due to space and formatting issues.

The SDDP algorithm was run with 1 trial solution per iteration for a total of 3000 iterations. The choice of 1 forward scenario at each iteration is already in use for a long time in the DOASA software [54], and was also studied in [51], where it was shown that for a strategy of keeping a fixed number of scenarios during the iteration process, this seems to be the most efficient parametrization to compute SDDP cuts. In [51] it was also shown that a strategy of increasing the number of sampled scenarios during the iteration process can give even better results, but due to the lack of studies regarding its parametrization it was not used in this work. The choice of 3000 iterations in our experiments was the same as used in [51], which had the same modelling simplifications and showed that the optimality gap is usually stable by the 3000-th iteration, and that the improvement in the solution is not worth the computational effort after that iteration. However, this choice is not meant to be the same in other implementations, as the detail level can increase the problem complexity and, then, increase the number of iterations required to get a reasonable solution. Another important point to mention is that, with a fixed number of iterations, the comparison among the cases are done in a fair basis, as all approaches have the possibility to calculate the same number of cuts. Figure 7.1 shows the lower bound convergence during the 3000 iterations. It is clear that the Backward independent cases stabilize before the dependent ones, and that the later ones stabilize after 2500-th iteration. Figure 7.2 shows the simulation costs in each forward simulation (with 1 scenario). The results of the risk averse cases are clearly more expensive than in the risk neutral one, as it was expected.

The scenarios tree used in the case studies had 100 realizations per stage with the total number of scenarios $1 \times 100 \times \cdots \times 100=100^{119}$. As a consequence, the backward procedure used 100 scenarios at each stage. For each approach, at the end of the iteration process two extra forward simulations were done, one with 2000 out-of-sample randomly generated scenarios using the periodic autoregressive model and another with 75 historical inflows. Most comparisons are shown using the generate scenarios, but in some cases in which 


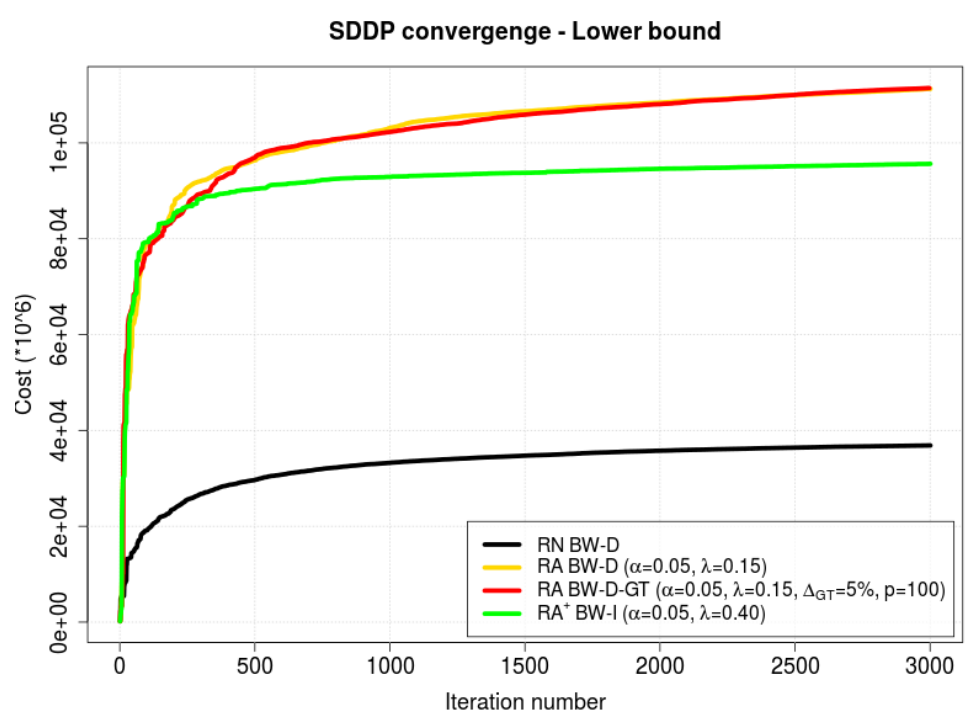

Figure 7.1: Lower bound over the iteration process

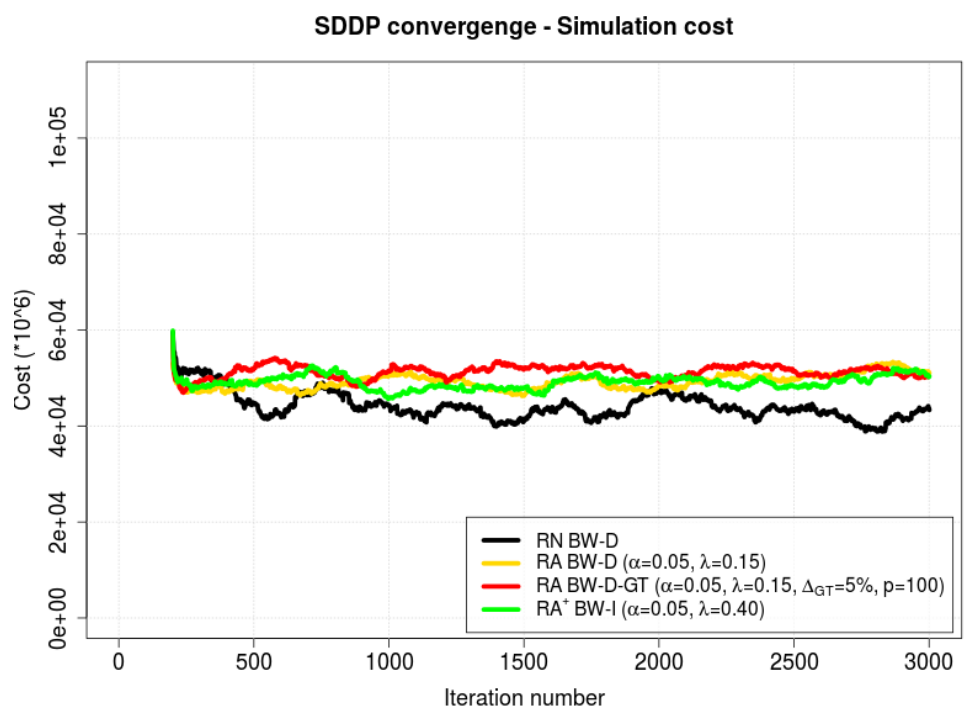

Figure 7.2: Simulation cost over the iteration process (cost for 1 forward scenario)

specific behaviour of some series are the focus of the analysis the historical scenarios are shown.

\section{2}

\section{Comparison between chosen case studies}

In this section we are going to compare both risk neutral and risk averse cases with the solutions of each proposed approach. In order to have a comparison of all solutions at a glance we are going to present the summary indexes first, followed by more detailed results regarding solution variability, cost and security. 


\subsection{1}

\section{Summary results}

The summary indexes results for the case studies are shown in Table 7.5. Although all of them should be minimized, they represent conflicting objectives. The reference case, "RA BW-D", is shown in bold. While the risk neutral case shows the best results for expected total cost, it does not show good results for system security and solution variability. The proposed approach "RA BW-D-GT" shows the best results for system security, but the worst for expected total cost criterion. Also, it does not show good results regarding solution variability, which in this case is only related to PLDs. The proposed approach "RA+ BW-I" shows the best results considering all criteria together. Although it does not show good results for cost-based criterion if compared to RN BW-D case, it is the best among the risk averse cases. Moreover, its system security results are similar to the other risk averse cases (although it is more than twice the value found by the other risk averse cases, all average risks over 5 years are lower than $1 \%$ ), and the variability criterion is best performed by this approach, suggesting that this is the best approach within the studied options. The increase in the cost for all risk averse cases can be seen as a premium paid to increase security of the solution.

\begin{tabular}{c|c|c|c} 
Case study & $\begin{array}{c}\text { Cost index } \\
\left(\times 10^{6}\right)\end{array}$ & $\begin{array}{c}\text { Security index } \\
(\%)\end{array}$ & $\begin{array}{c}\text { Variability index } \\
(\mathrm{R} \$ / \mathrm{MWh})\end{array}$ \\
\hline RN BW-D & $40,300.79$ & 2.26 & 261.44 \\
RA BW-D & $\mathbf{4 9 , 0 3 5 . 3 4}$ & $\mathbf{0 . 2 3}$ & $\mathbf{2 3 4 . 7 7}$ \\
RA BW-D-GT & $49,862.23$ & 0.20 & 239.78 \\
RA $^{+}$BW-I & $47,944.02$ & 0.48 & 188.71
\end{tabular}

Table 7.5: Summary results indexes

These results give an overview of the solutions obtained with each approach to solve the problem, and show that the objective of this work is accomplished. A more detailed analysis of the results is given in the ensuing text.

\subsection{2}

\section{Solution variability indexes}

The proposal of this work is to reduce the SDDP solution uncertainty and variability, and the investigation of this topic is the main objective of this section.

As mentioned before - and despite not being considered in the problem formulation - one could favour thermal generation decisions that do not change very much on a weekly basis, as it may imply in fuel logistic difficulties and 
in higher total operation cost for the system along the planning horizon. Moreover, as a long-term operation problem, the weekly decisions shouldn't be so strongly based on recent inflows information.

\section{Solution uncertainty index: 1-SAFE measure}

The first results are focused on the measurement of the volatility reduction over time, which is directly related to the predictability of the solution. This volatility was assessed using the 1-SAFE measure, which methodology is described in Section 6.1.1. Figures 7.3 and 7.4 depict the 1-SAFE index over time, and it is clear that the proposed methodologies are able to reduce the solution volatility, with RA BW-D-GT reducing only thermal generation volatility, while $\mathrm{RA}^{+}$BW-I reduces also PLD volatility.

This result is very important, because it shows that the solution predictability is increased by the proposed approaches and, if the remaining characteristics of the solution are not deteriorated, implies that the main objective of this work is achieved. However, before assessing the other criteria of interest, let us first evaluate the solution variability and uncertainty with respect to different measures.

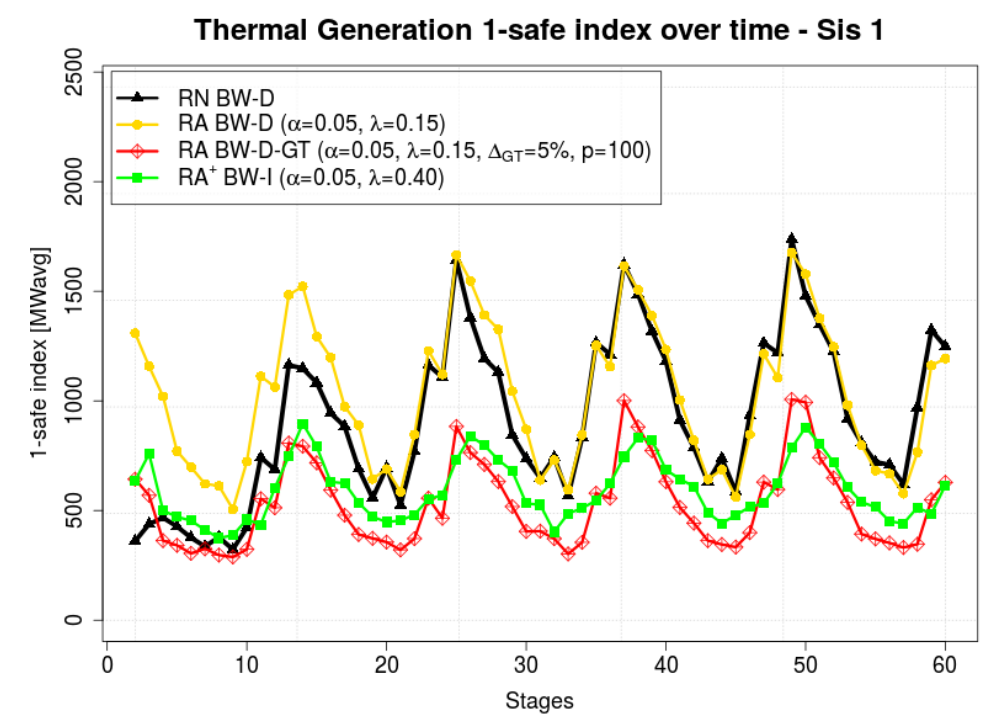

Figure 7.3: Thermal Generation volatility 1-SAFE measure over time

\section{Solution uncertainty at each stage}

The second results, presented in Figure 7.5, depicts the average thermal generation and the $5 \%$ and $95 \%$ quantiles, and show that the thermal generation uncertainty at each stage is reduced in both proposed approaches, although in RA BW-D-GT proposal the reduction is not very significant. Ad- 


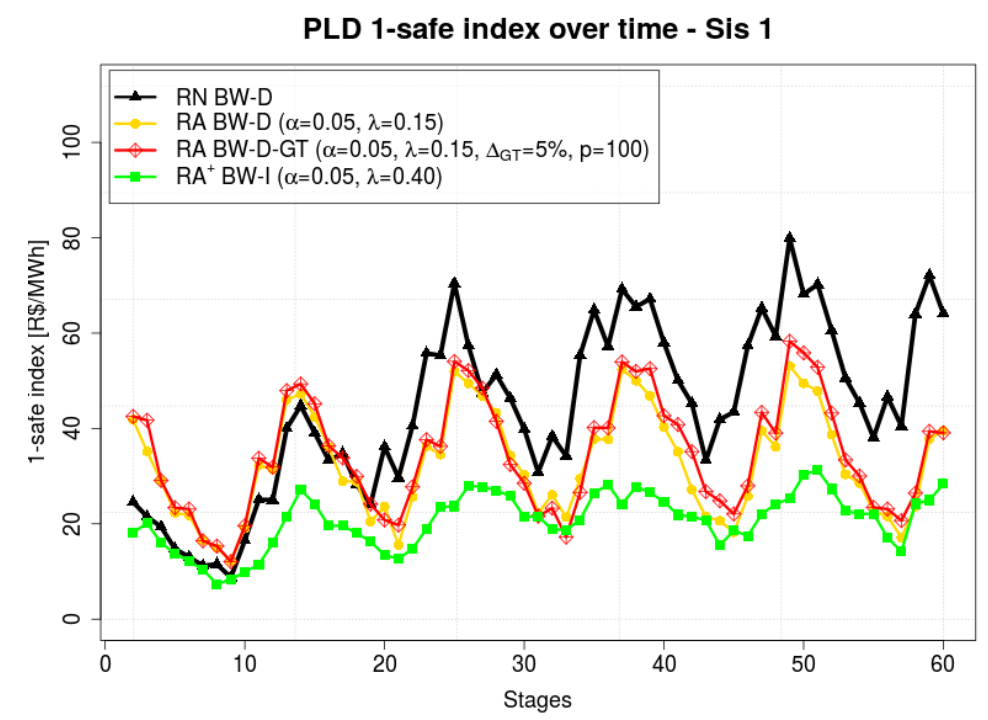

Figure 7.4: PLD volatility 1-SAFE measure over time

ditionally, Figure 7.6 shows that the uncertainty of PLDs distribution at each stage is reduced only in $\mathrm{RA}^{+} \mathrm{BW}-\mathrm{I}$ approach. This result reinforces the appropriateness of the presented proposals, and shows that the uncertainty regarding the solution can be reduced.

Thermal Generation for each stage - Q5\%, Q95\% and mean - Sis 1

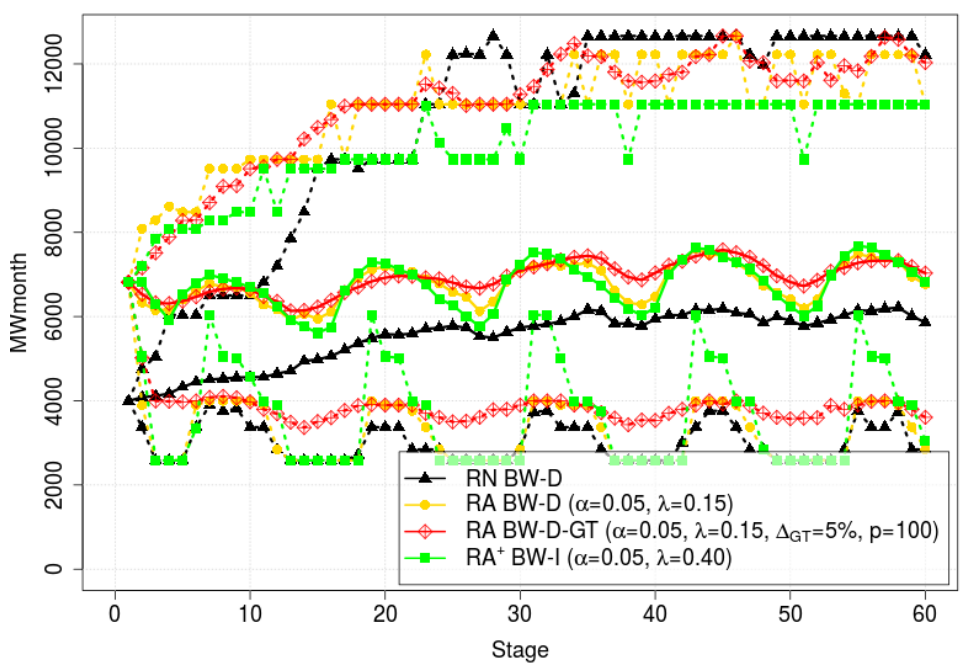

Figure 7.5: Thermal Generation average and 5\% and 95\% quantiles

An uncertainty index can be calculated by the average $90 \%$ empirical confidence interval for both variables, and is shown in Table 7.6. This index was already shown for PLDs in the summary results. 


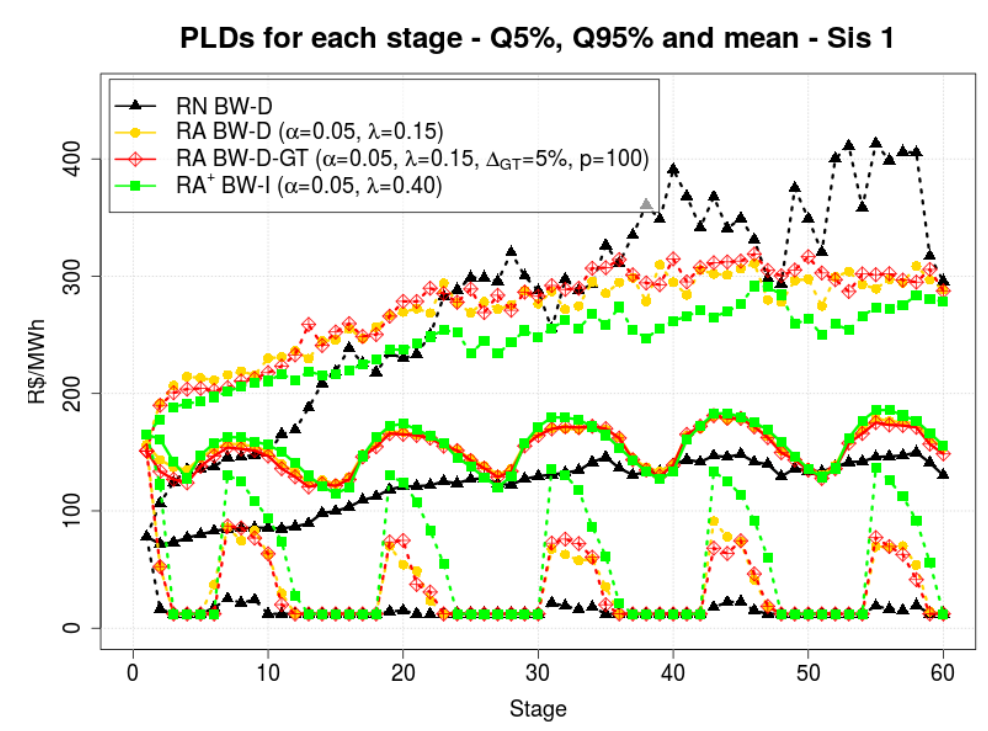

Figure 7.6: PLDs average and 5\% and 95\% quantiles

\begin{tabular}{c|c|c} 
Case study & $\begin{array}{c}\text { Thermal generation } \\
(\text { MWavg })\end{array}$ & $\begin{array}{c}\text { PLD } \\
(\mathrm{R} \$ / \mathrm{MWh})\end{array}$ \\
\hline RN-BW-D & 7533.70 & 261.44 \\
RA-BW-D & 7633.50 & 234.77 \\
RA-BW-D-GT & 7115.70 & 239.78 \\
RA $^{+}$BW-I & 6333.45 & 188.71
\end{tabular}

Table 7.6: Uncertainty indexes

\section{Solution variability over time}

The next result we are going to show in this section is related to the variability of thermal generation over time for a given scenario. The procedure used to calculate this index was described in Section 6.1. The distribution of the variability index $V_{s}$ for thermal generation is shown in Figure 7.7. It is clear that both proposed approaches imply in a reduction of thermal generation variability over time. It is important to note that in the $\mathrm{RA}^{+} \mathrm{BW}-\mathrm{I}$ case this result is uncontrollable, while in the RA BW-D-GT case the adjustment of the penalty value and $\Delta_{G T}$ allows the decision makers to fine tune the variability over time.

This result is summarized by its mean and standard deviation for all systems in Table 7.7 and Table 7.8. It is possible to notice that not only the expected variability, but also its standard deviation, is reduced in all systems but North. The exception of the North system is related to its very small thermal generation capacity, with only two thermal plants in this case study, with installed capacity of 166 MW each - note that for the North system the total installed capacity is $7740 \mathrm{MW}$.

The reduction in thermal generation variability is also illustrated by 


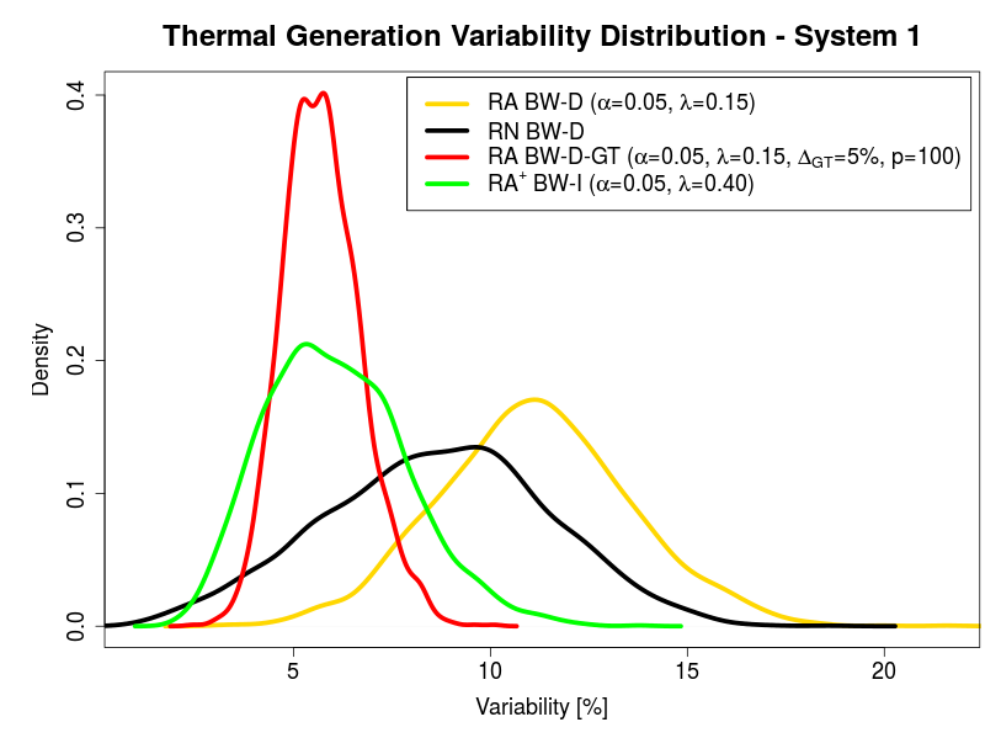

Figure 7.7: Thermal generation variability

\begin{tabular}{c|c|c|c|c} 
& RN BW-D & RA BW-D & RA BW-D-GT & RA $^{+}$BW-I \\
\hline Southeast & 8.64 & 11.13 & 5.72 & 6.09 \\
South & 13.66 & 17.74 & 7.10 & 10.39 \\
Northeast & 17.13 & 14.58 & 6.77 & 7.53 \\
North & 5.51 & 2.61 & 3.93 & 3.45
\end{tabular}

Table 7.7: Thermal Generation Average Variability

\begin{tabular}{c|c|c|c|c} 
& RN BW-D & RA BW-D & RA BW-D-GT & RA + BW-I \\
\hline Southeast & 2.84 & 2.43 & 0.98 & 1.79 \\
South & 5.06 & 6.25 & 2.25 & 4.88 \\
Northeast & 5.86 & 4.89 & 2.32 & 3.15 \\
North & 42.54 & 6.62 & 26.41 & 7.60
\end{tabular}

Table 7.8: Thermal Generation Variability Standard Deviation

Figure 7.8, which shows, for a given scenario, the thermal generation behaviour over monthly stages for a simulation with a historical scenario. The chosen scenario was the period between 2001 and 2005, which comprehends Brazilian energy rationing occurred between 2001 and 2002.

The same procedure described in Section 6.1 was applied to PLDs, and its variability index was calculated for all scenarios. The distributions of variability indexes $V_{s}$ for PLDs are shown in Figure 7.9. Unlike seen in thermal generation results, where both proposed approaches implied in a reduction of thermal generation variability, only the $\mathrm{RA}^{+}$BW-I proposal resulted in a reduction of PLDs variability over time. This result was expected, since the RA BW-D-GT approach is aimed just at controlling thermal generation variability over time.

This result is emphasized for all systems in Table 7.9 and Table 7.10, where only $\mathrm{RA}^{+} \mathrm{BW}-\mathrm{I}$ case shows a reduction on both average and standard deviation for all systems. 


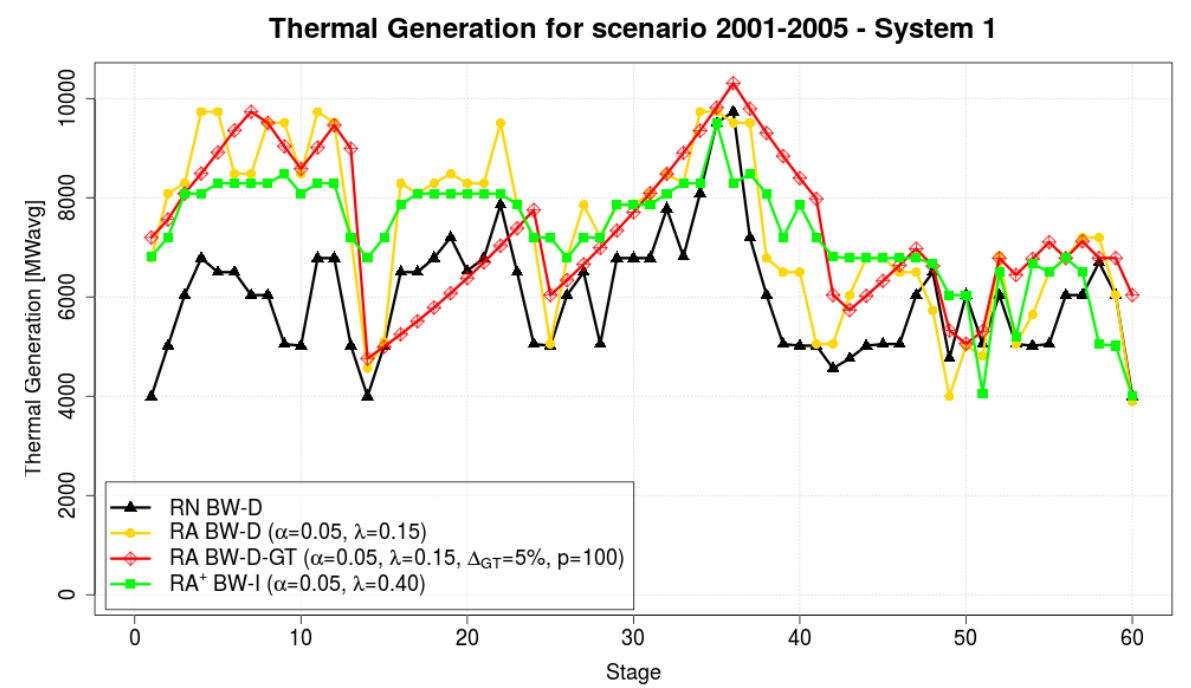

Figure 7.8: Thermal generation for simulation with historical inflows occurred between 2001-2005

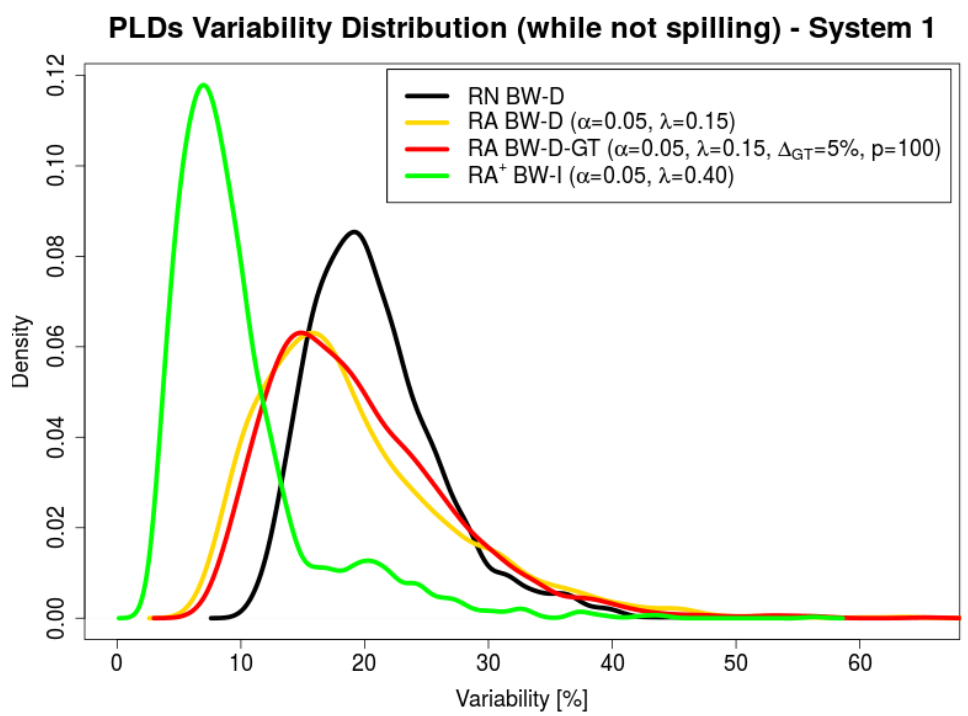

Figure 7.9: PLD variability

The previous results are illustrated for a particular historical scenario 2001-2005 in Figure 7.10, where the PLDs over time are shown.

\section{Solution variability given the same initial conditions}

The last set of results regarding solution variability that is going to be shown is the second stage thermal generations distribution. The "RA BW-D $(\lambda=0.15, \alpha=0.05)$ " case is the reference on Figure 7.11, and is shown by the blue histogram. On top of Figure 7.11 we can see that the variability of thermal generations in the second stage is higher in RA BW-D case, with a high frequency of thermal dispatches far from first stage solution, while in the risk 


\begin{tabular}{c|c|c|c|c} 
& RN BW-D & RA BW-D & RA BW-D-GT & RA + BW-I \\
\hline Southeast & 21.62 & 19.27 & 19.66 & 9.99 \\
South & 21.88 & 19.05 & 20.60 & 9.48 \\
Northeast & 25.45 & 21.09 & 22.12 & 11.64 \\
North & 30.55 & 24.26 & 25.38 & 15.03
\end{tabular}

Table 7.9: PLD Average Variability

\begin{tabular}{c|c|c|c|c} 
& RN BW-D & RA BW-D & RA BW-D-GT & RA $^{+}$BW-I \\
\hline Southeast & 6.16 & 8.05 & 7.41 & 5.99 \\
South & 6.18 & 7.82 & 7.52 & 5.47 \\
Northeast & 6.98 & 7.72 & 7.23 & 4.96 \\
North & 12.90 & 12.53 & 10.22 & 7.98
\end{tabular}

Table 7.10: PLD Variability Standard Deviation

PLD for scenario 2001-2005 - System 1

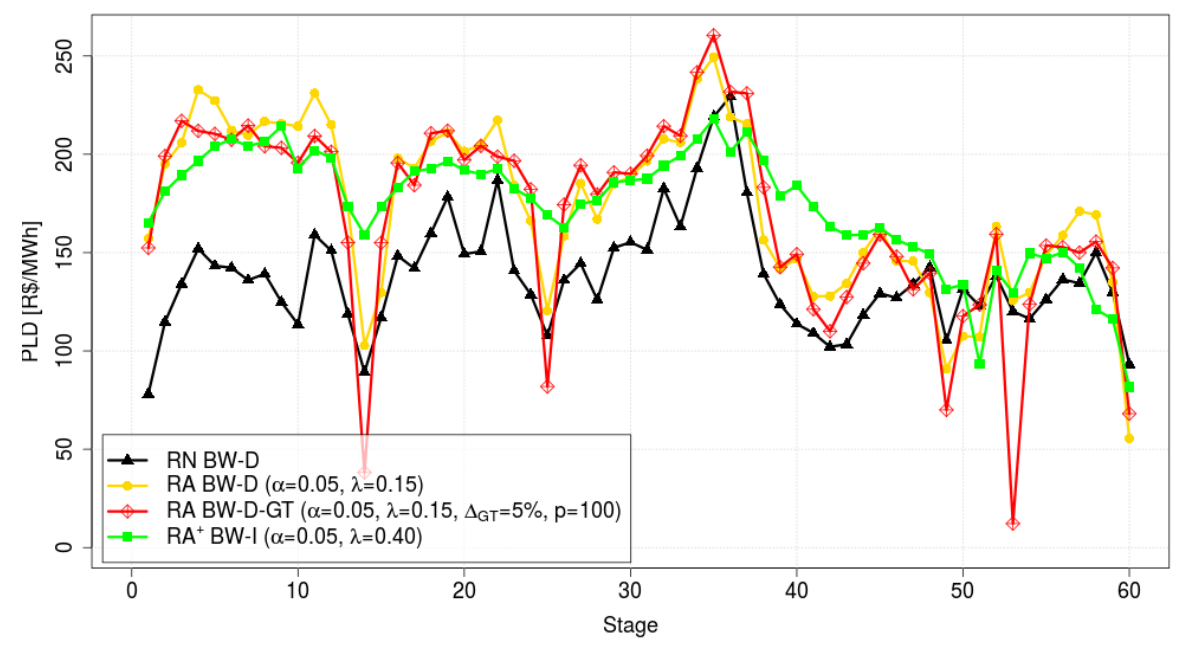

Figure 7.10: PLDs for simulation with historical inflows occurred between 20012005

neutral case this variability is lower due to smaller dispatch values. On the other hand, on both proposed approaches, on the pictures at the bottom of Figure 7.11, the second stage variability is much smaller and concentrated near the first stage value. Some summary statistics of second stage thermal generation are shown in Table 7.11. The average and maximum thermal dispatch is greater in risk averse cases, and the minimum is similar in first three cases and equals to the inflexibility of thermal generators, while in RA BW-D-GT case it has a bit higher value. As a consequence of a higher average while still presenting low dispatch values in many scenarios, the standard deviation of risk averse cases are higher than risk neutral one. On the other hand, comparing the standard risk averse case with proposed approaches, there is a reduction in the standard deviation and in the maximum value.

The same kind of results are shown for second stage PLDs, in Figure 7.12, and the summary statistics in Table 7.12 . We can see that PLDs distribution 

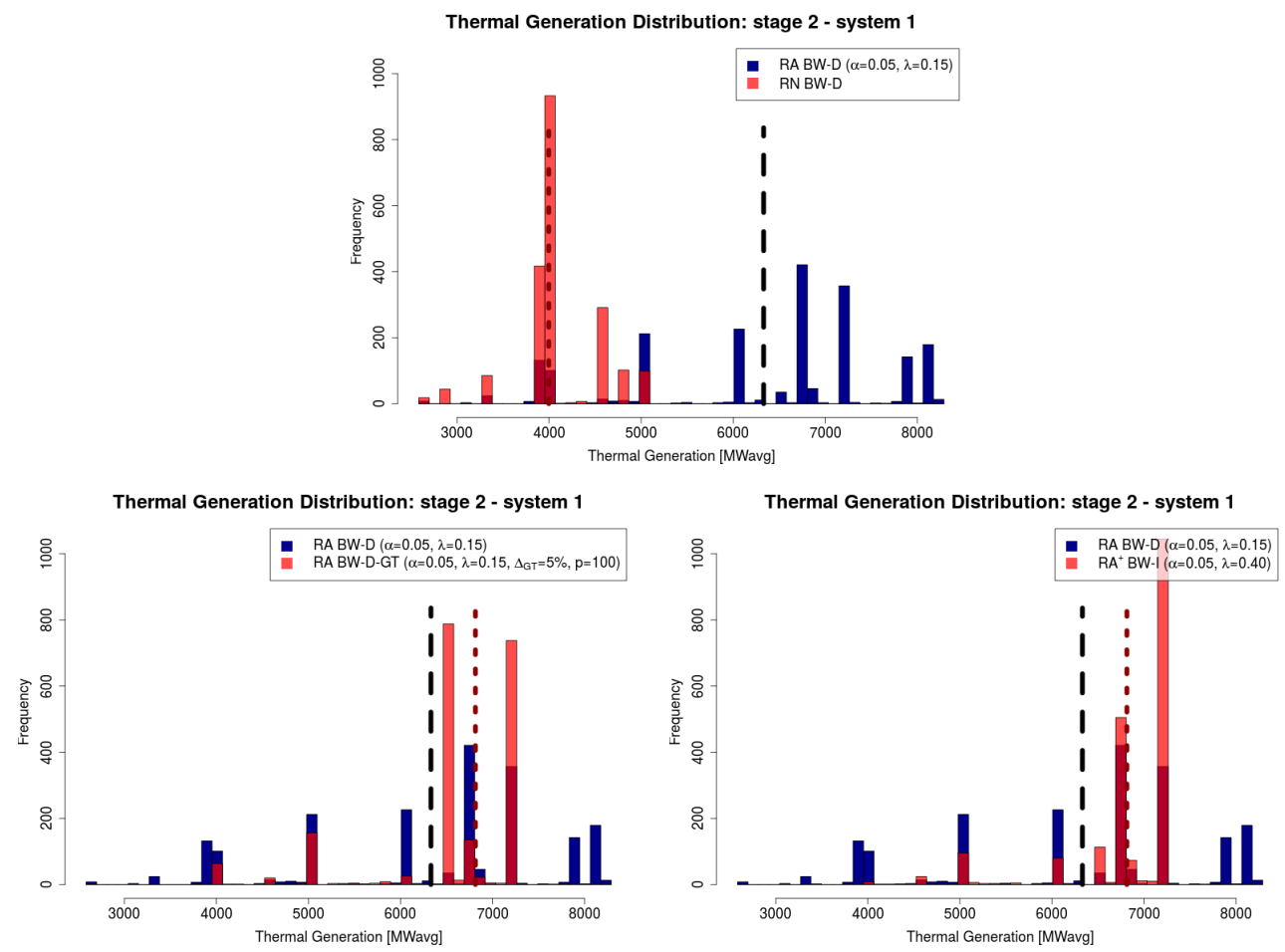

Figure 7.11: Second stage thermal generation [MWmonth]

\begin{tabular}{c|c|c|c|c|c} 
Case study & Std. Deviation & Average & Median & Minimum & Maximum \\
\hline RN BW-D & 432.00 & 4084.54 & 3996.40 & 2584.60 & 5018.40 \\
RA BW-D & 1335.95 & 6330.93 & 6784.20 & 2584.60 & 8290.20 \\
RA BW-D-GT & 765.83 & 6528.14 & 6500.20 & 3996.40 & 7200.20 \\
RA + BW-I & 642.02 & 6811.62 & 7200.20 & 2584.60 & 7200.20
\end{tabular}

Table 7.11: Second stage thermal generation statistics $[\mathrm{R} \$ / \mathrm{MWh}]$

of the proposed approach $\mathrm{RA}^{+}$BW-I are much more concentrated around the first stage value and that its standard deviation and maximum value are smaller than the standard risk-averse approach. The proposed approach RA BW-D-GT does not show good results for PLDs, as already mentioned in this work.

\section{Conclusions}

The main conclusion of this subsection is that it is possible to reduce the solutions uncertainty and variability over time with both proposed approaches. While the first proposal deals only with the thermal generation behaviour, the second proposal allows the stabilization of both thermal generation and PLDs. This characteristic makes the "RA+ BW-I" a better choice to stabilize SDDP solutions in the energy operation planning framework. In order to assess other properties of the proposed approaches and to check whether the same properties of the reference case "RA BW-D" still hold in these alternatives, the next section will investigate the cost based indexes that were presented in 

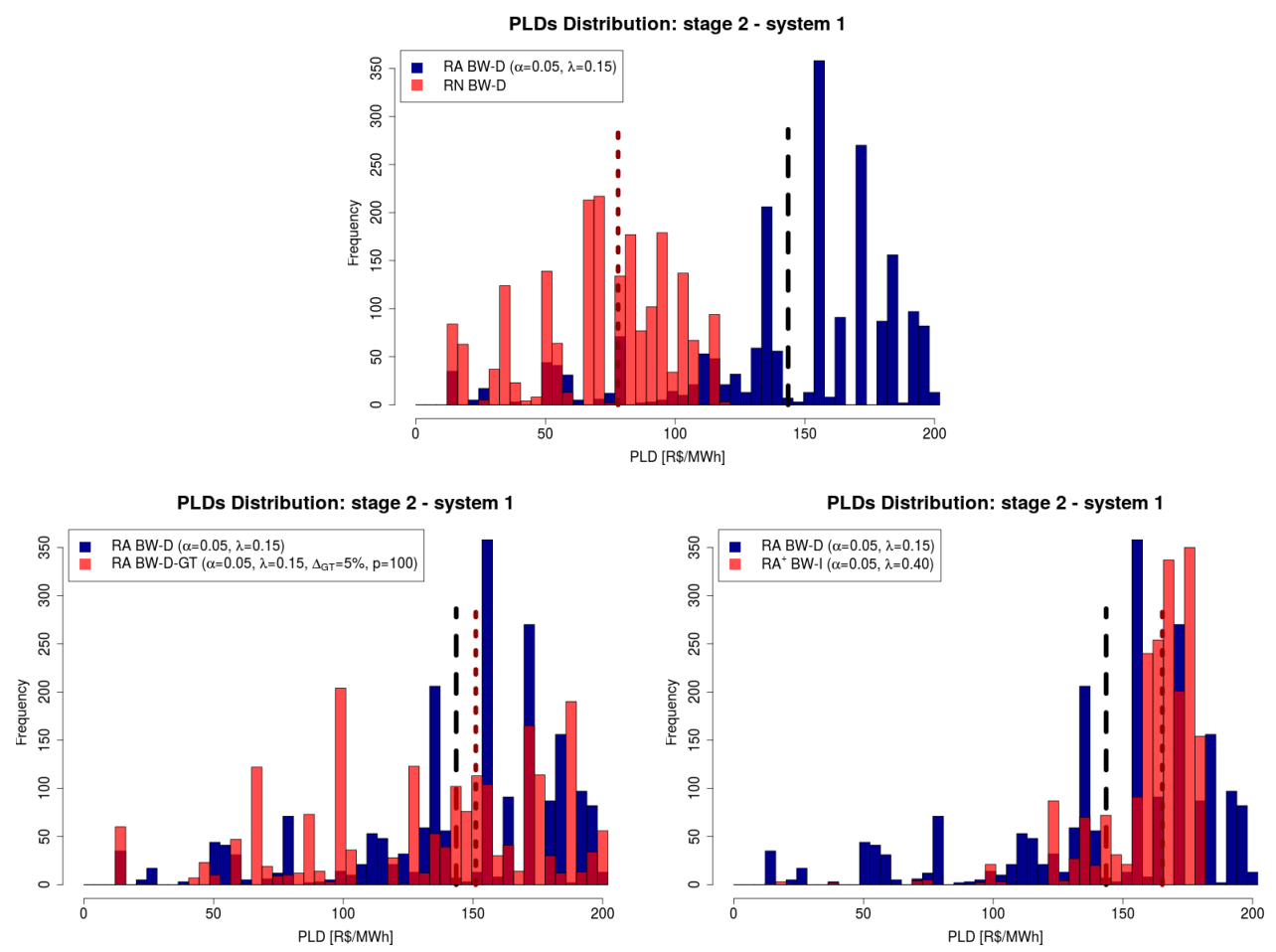

Figure 7.12: Second stage PLDs

\begin{tabular}{c|c|c|c|c|c} 
Case study & Std. Deviation & Average & Median & Minimum & Maximum \\
\hline RN BW-D & 27.93 & 71.42 & 77.60 & 0.00 & 118.10 \\
RA BW-D & 43.24 & 143.48 & 156.60 & 0.00 & 201.50 \\
RA BW-D-GT & 47.18 & 133.43 & 144.20 & 0.00 & 202.00 \\
RA $^{+}$BW-I & 18.49 & 161.11 & 166.60 & 0.00 & 181.10
\end{tabular}

Table 7.12: Second stage PLDs statistics

Section 6.2.

\subsection{3}

\section{Cost-based indexes}

Besides being able to reduce the solution uncertainty, the proposed approaches must be able to find solutions similar to the ones obtained by the RA BW-D case with respect to the simulations costs and systems security. The following sections investigate whether these characteristics are kept by the proposed approaches.

\section{Simulation costs}

The operation costs for each stage are shown in Figure 7.13. The thicker continuous lines are the average costs for a 2000 scenarios simulation and the dotted lines show the $99 \%$ quantiles of the individual stages cost. We can see that the risk averse cases results are very similar and that there is a reduction in the cost peaks with a small increase in the average cost. This is a first 
evidence that the proposed approaches are able to give solutions as good as the standard risk-averse SDDP.

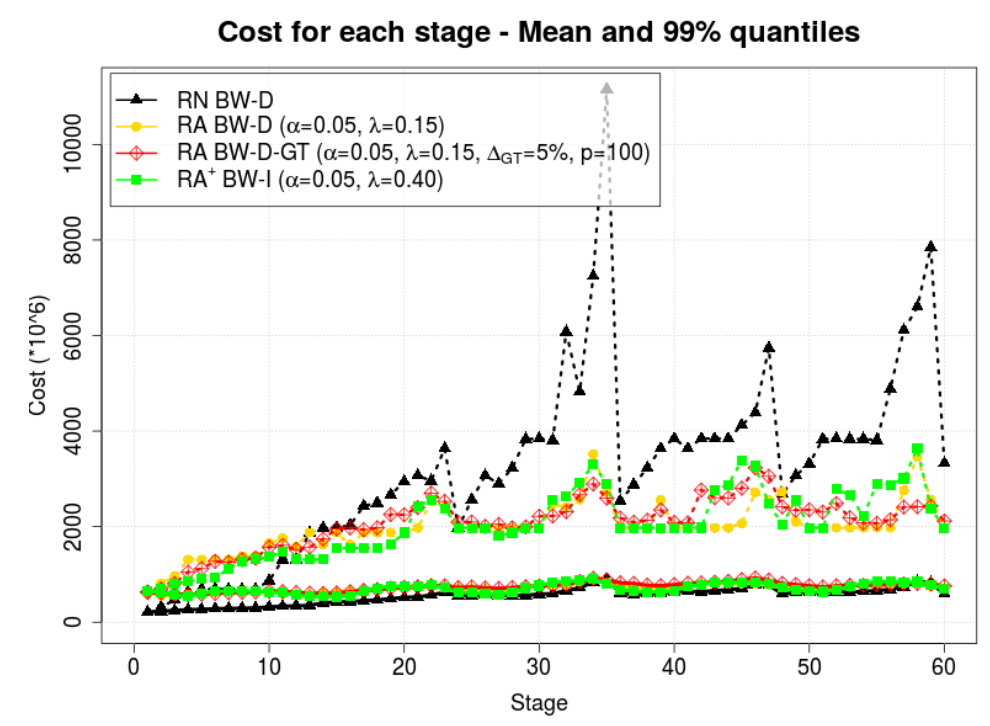

Figure 7.13: Average and 99\% quantile costs for each stage

\section{Policy values}

The second cost-based index is the resulting average policy values for 2000 scenarios and is shown in Table 7.13 together with its $95 \%$ confidence interval upper and lower bounds. The results for the RA BW-D-GT approach, although slightly higher than the standard risk averse case, shows an intersection between their average confidence interval. This difference may be explained by the slightly safer solution, and then using more thermal dispatch, given by this approach in comparison with the RA BW-D case. The same applies to the $\mathrm{RA}^{+} \mathrm{BW}-\mathrm{I}$ case, which has a lower value than RA BW-D case, and which found a solution that is less safe than the one found by RA BW-D case. Although the $\mathrm{RA}^{+}$BW-I has a higher risk aversion than the RA BW-D cases, this increased risk aversion is working as an ambiguity aversion, and is not necessarily expected to give higher costs in comparison to the other risk averse cases. In fact, the risk aversion level was increased in order to give, in conjunction to the lack of information in the backward scenarios, a similar risk aversion level as the RA BW-D cases and, then, similar policy values.

\section{Conditional Value at Risk}

The conditional value at risk results are consistent with the expected results, that is, the RN BW-D shows the worst result, which means that the average cost of the $5 \%$ most expensive scenarios in this approach is much higher 


\begin{tabular}{c|c|c|c} 
Case study & $\begin{array}{c}\text { LB CI } \\
(95 \%)\end{array}$ & $\begin{array}{c}\text { Average } \\
\text { Policy value }\end{array}$ & $\begin{array}{c}\text { UB CI } \\
(95 \%)\end{array}$ \\
\hline RN BW-D & $39,229.02$ & $40,300.79$ & $41,372.57$ \\
RA BW-D & $48,306.27$ & $49,035.34$ & $49,764.40$ \\
RA BW-D-GT & $49,151.69$ & $49,862.23$ & $50,572.77$ \\
RA $^{+}$BW-I & $47,187.80$ & $47,944.02$ & $48,700.25$
\end{tabular}

Table 7.13: Policy values $\left[\mathrm{R} \$ \times 10^{6}\right]$

than in cases where the CV@R is taken into account in the objective function. Moreover, the best results were achieved by RA BW-D case, in which the only objective is to minimize total expected cost and CV@R.

\begin{tabular}{c|c|} 
Case study & CV@R \\
\hline RN BW-D & $115,487.90$ \\
RA BW-D & $92,195.88$ \\
RA BW-D-GT & $94,611.50$ \\
RA ${ }^{+}$BW-I & $96,972.39$
\end{tabular}

Table 7.14: $5 \%$ Conditional Value at Risk $\left[\mathrm{R} \$ \times 10^{6}\right]$

\section{Conclusions}

The results shown in this section makes clear that the proposed approaches have the same cost behaviour as the reference case. Moreover, the "RA ${ }^{+}$BW-I" was able to achieve similar results with a smaller expected cost. The only result which the proposed approaches have underperformed the reference case was the CV@R one, although the reduction compared to the risk neutral case was significant.

\subsection{4}

\section{Operation security indexes}

\section{Stored volumes}

In hydrothermal operation planning problem, a conservative solution may be characterized as the one in which thermal plants are dispatched more frequently, specially in dry inflows periods. This means that the hydro plants are used with more caution and the stored volumes are kept in higher levels aiming at reducing the costs during dry seasons. This behaviour is shown in Figure 7.14, where all risk averse cases have higher stored volumes (on average - continuous line - and on 5\% and 95\% quantiles - dashed lines). Another behaviour that can be observed is that the RA BW-D and RA BW-D-GT cases show higher $5 \%$ quantile and lower $95 \%$ quantile if compared to the $\mathrm{RA}^{+}$BW-I approach. This can be explained by the past inflows dependent characteristic taken into account by backward dependent policy approaches. In 
critical situations it rises thermal generation, and hence, tries to keep reservoirs levels high, while in wet situations it decreases thermal generation and reduces the stored volumes. This characteristic is intuitive and seems to be correct, but, due to expectation reversals, it increases solution variability, which ultimately cause undesired effects in the operation planning and its related areas.

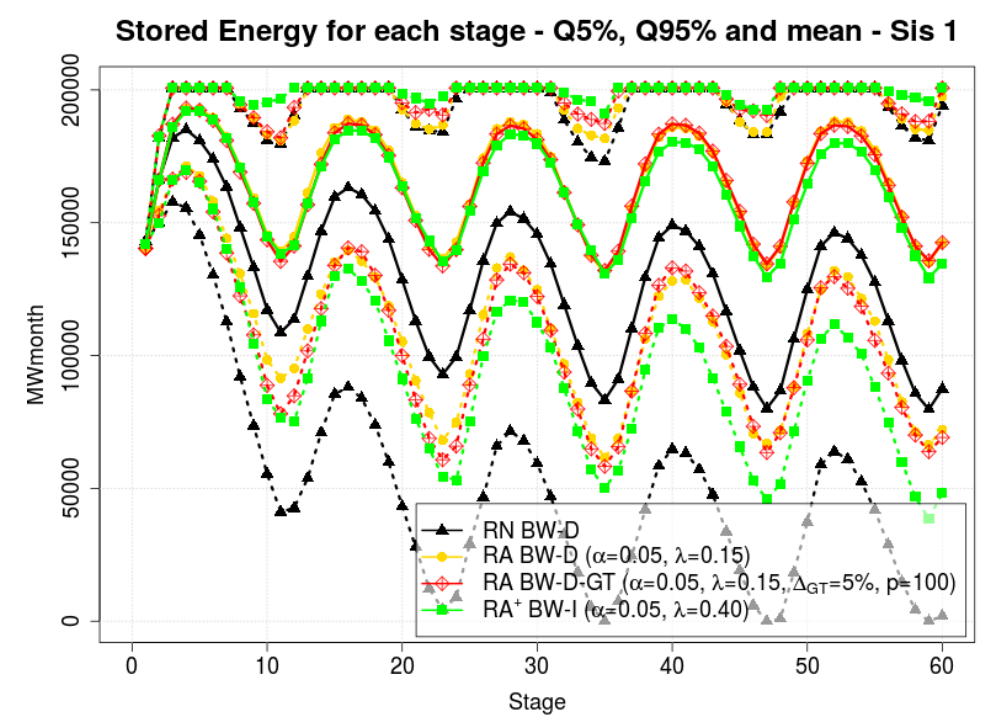

Figure 7.14: Average, 5\% and 95\% quantile stored volumes for each stage [MWmonth]

Although it is possible to see that the proposed approaches are very similar to standard risk averse case, this analysis lacks a more objective comparison of the stored energy for all alternatives. In order to make this comparison more objective we have calculated the stored energy quality indexes presented in Chapter 6, which distinguishes between solutions that are better in dry and wet situations, for example. The quality index for each stage for the total stored volume (sum of all systems) is shown in Figure 7.15, from which we conclude that standard risk averse and RA BW-D-GT cases are better in almost every stage, with $\mathrm{RA}^{+} \mathrm{BW}-\mathrm{I}$ coming on third, and the risk neutral case with very poor results.

Moreover, the results for all systems (shown in Table 7.15) are standardized with respect to the best solution for each system to simplify our analysis process. In this way, the best solution for each system always appears as 1.00. The standard RA BW-D case shows the best performance for all systems except for the South, where the $\mathrm{RA}^{+}$BW-I performed best. The fact $\mathrm{RA}^{+} \mathrm{BW}-\mathrm{I}$ was better in South can be explained by the high variability and low stagewise correlation of the inflows in this system, which makes an independent approach as good as a dependent one. Moreover, the proposed approaches have scores greater than 0.90 in all systems, which means that even in situations where 


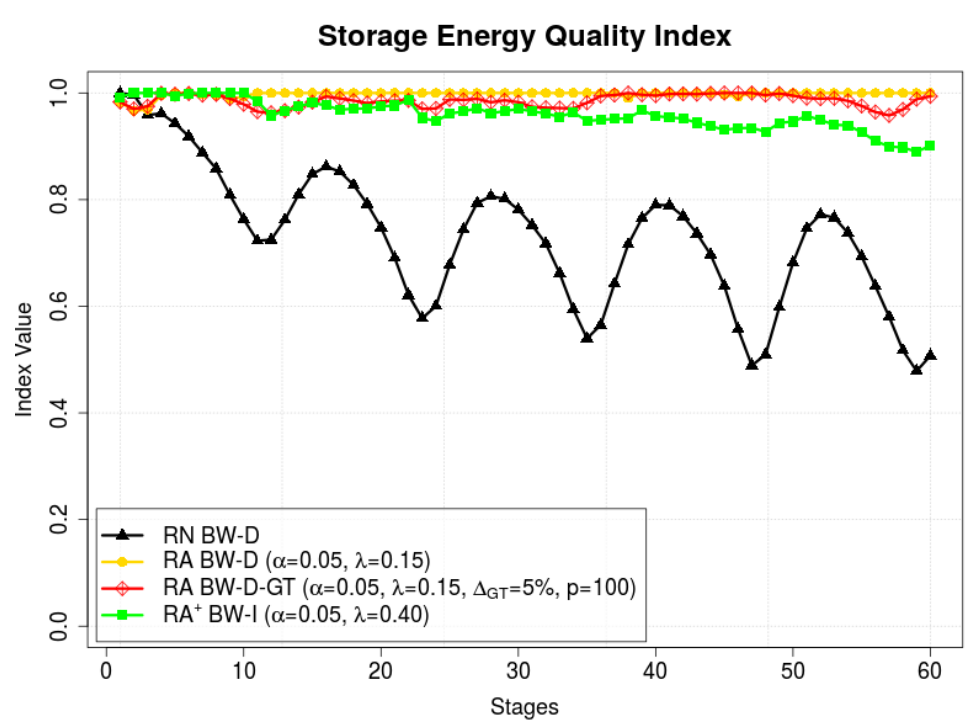

Figure 7.15: Stored Energy Quality Index for each stage

the proposed approaches don't have the best performance, the results are not far from the best solution.

\begin{tabular}{c|c|c|c|c} 
& RN BW-D & RA BW-D & RA BW-D-GT & RA $^{+}$BW-I \\
\hline Southeast & 0.709 & 1.000 & 0.991 & 0.961 \\
South & 0.569 & 0.881 & 0.925 & 1.000 \\
Northeast & 0.662 & 1.000 & 0.933 & 0.976 \\
North & 0.831 & 1.000 & 0.911 & 0.953
\end{tabular}

Table 7.15: Stored Energy Quality Indexes

\section{Risks of deficit}

Although the reduction of cost peaks may indicate a more conservative system operation, it is very important to certify that the risks of deficit are kept below certain levels. In Figure 7.16 we show the Southeast annual risks of deficits greater than $1 \%, 5 \%, 10 \%$ and above $20 \%$ of demand. We can see that the proposed approaches are able to reduce the risks of deficits greater than $1 \%$ to a level below $1 \%$. Moreover, the RA BW-D-GT case shows risks that are similar to the ones observed in the standard risk averse case.

\section{Discussion on the solution security}

Although the previous results show that the security level obtained by the proposed approaches are similar to the ones observed in the standard risk averse SDDP, one may argue that in critical situations the operation calculated by the standard RA BW-D SDDP and the proposed approach RA BW-D-GT are much more secure due to the consideration of the autoregressive model in the backward procedure. 


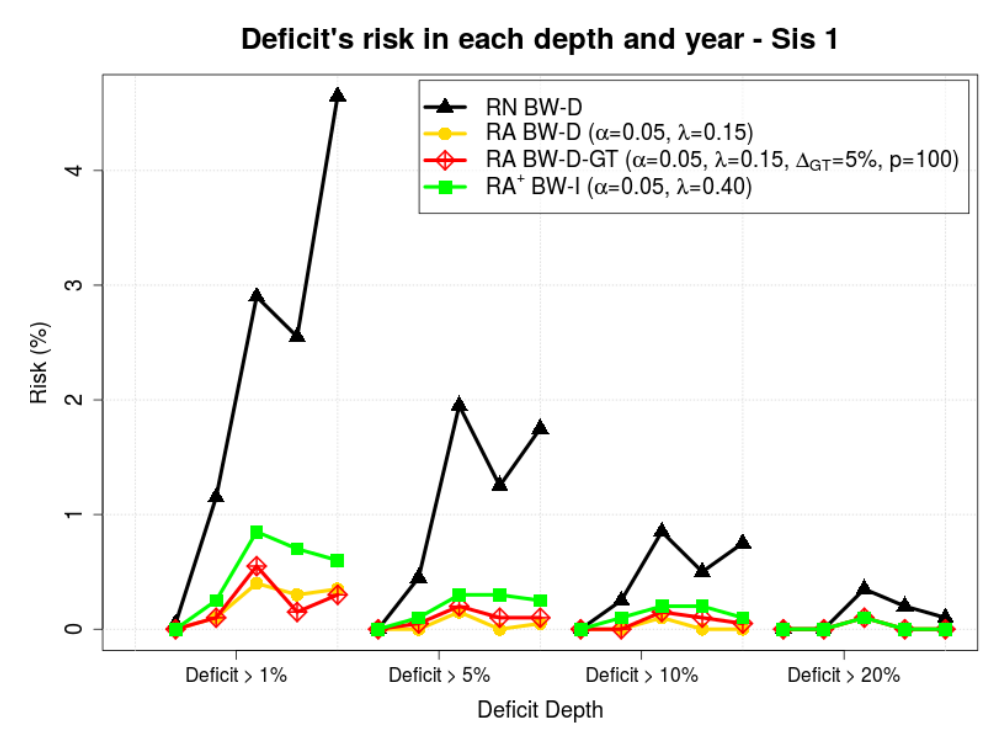

Figure 7.16: Annual risks of deficit [\%]

In order to assess whether this claim is true, we compared the solution behaviour of each approach in several hydrological situations: wet, regular and dry. The results are aggregated for all systems, that is, represents the sum of the results for SE, S, NE and N.

- Wet hydrological condition: this situation is exemplified by the historical scenario occurred between 1979-1983, which was extremely wet in comparison to the rest of the historical record. The stored energies for a simulation with such scenario are shown in Figure 7.17, and show that the $\mathrm{RA}^{+}$BW-I approach keeps the reservoir in a higher level, as expected due to the already shown results. In such a wet scenario, we could expect that this solution approach would result in a higher spillage if compared to the backward dependent approaches. However, this is not observed, as shown in Figure 7.18. Although the stored level is higher in the $\mathrm{RA}^{+} \mathrm{BW}-\mathrm{I}$ case, actually the reservoir level recovery of $\mathrm{RA}^{+} \mathrm{BW}-\mathrm{I}$ case is slower than the recovery observed on the other approaches, and the maximum capacity is often reached around the same stage.

- Regular hydrological condition: to exemplify this condition the period between 2004 and 2008 was chosen. There is no clear bias on the $\mathrm{RA}^{+}$BW-I solution (see Figure 7.19) and, with the exception of the last stages, the storage remains close the other solutions.

- Dry hydrological condition: this is the main objective of this analysis, and two different periods were chosen: 2001-2005, which comprises the Brazilian energy rationing, and 1952-1956, the most critical period so far in the Brazilian inflows record. Figure 7.20 shows the stored volumes, 


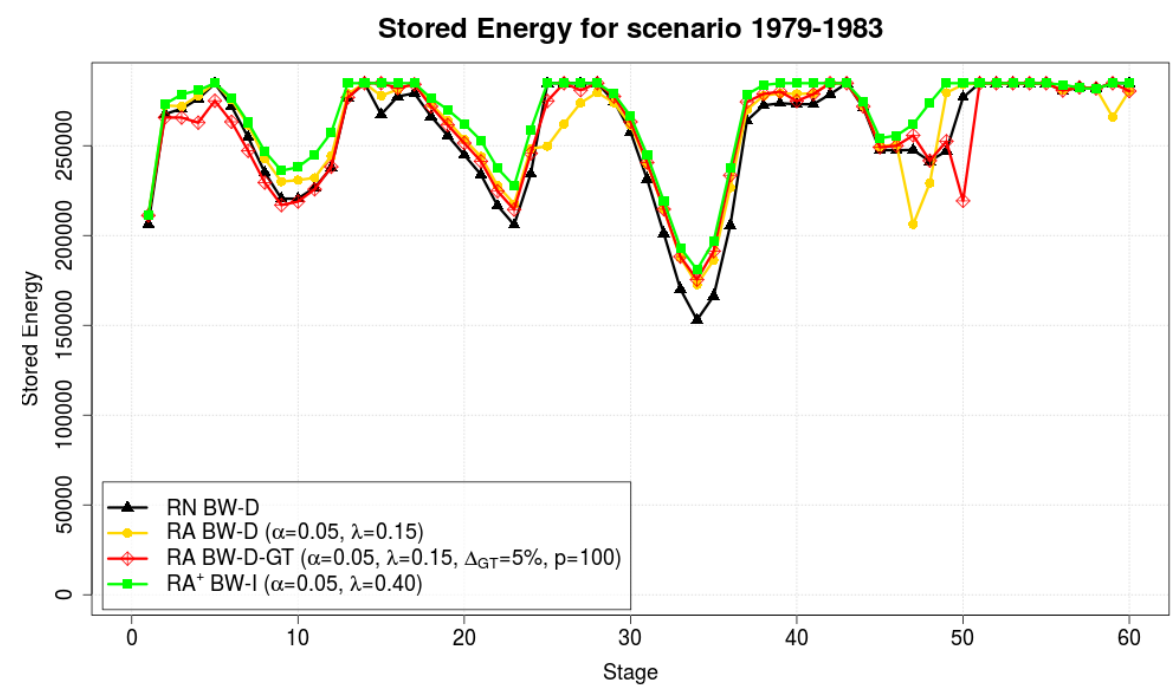

Figure 7.17: Total stored energy for simulation with historical inflows occurred between 1979-1983

Spilled Energy for scenario 1979-1983

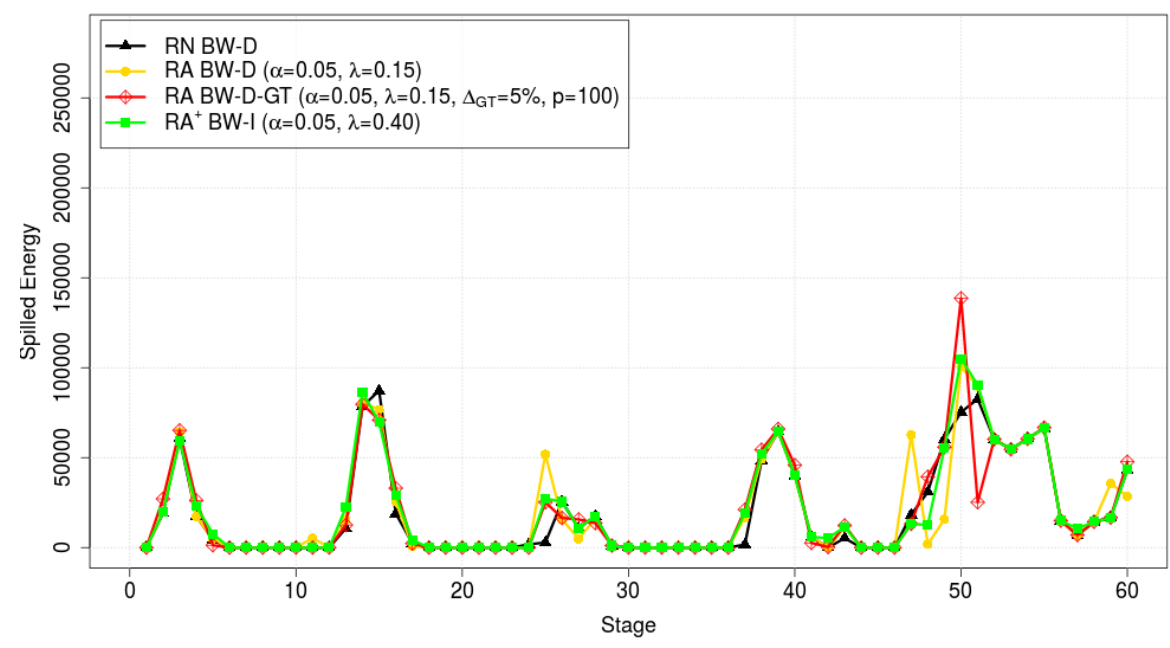

Figure 7.18: Total spilled Energy for simulation with historical inflows occurred between 1979-1983

and it is possible to see that all risk averse cases kept the stored levels much higher than the risk neutral case. In this simulation, no deficit was observed in any case. The next step is to assess the solution under the most extreme situation ever observed, the period between 1952-1956. As expected, Figure 7.21 shows that the $\mathrm{RA}^{+}$BW-I case gives a stored volume lower than the backward dependent cases, although they all end up with the same stored level. In order to clarify the impact of such a lower storage level, Figure 7.22 shows the total deficit for each case. It is not clear that the backward dependent cases are more secure than the independent one. Moreover, for practical purposes, once the stored 


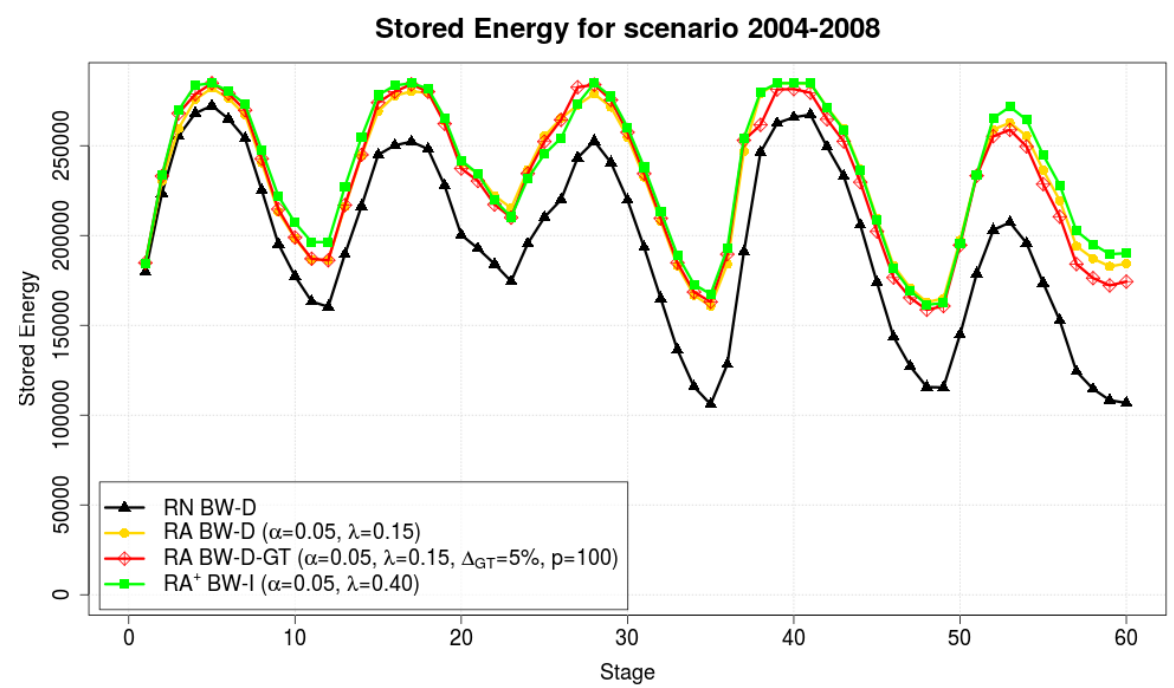

Figure 7.19: Total stored energy for simulation with historical inflows occurred between 2004-2008

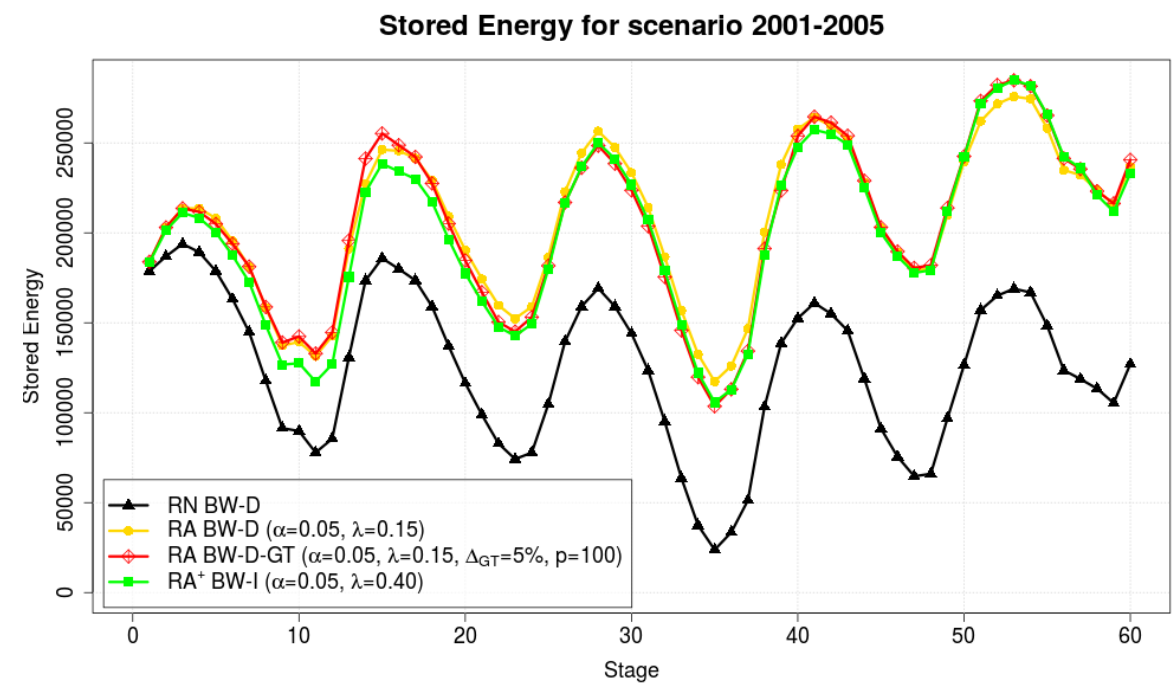

Figure 7.20: Total Brazilian stored energy for simulation with historical inflows occurred between 2001-2005

volumes achieve a very low level, the system operator would not allow the thermal plants to be turned off, what would reduce the chances of having such deficit spikes as observed in the picture.

\section{Conclusions}

The last criterion evaluated was the solutions security. The results show that both proposed approaches achieves the same security levels as the reference case, with "RA+ BW-I" case being slightly worse than "RA BW-DGT" case. However, this difference is negligible, and can be overlooked due 


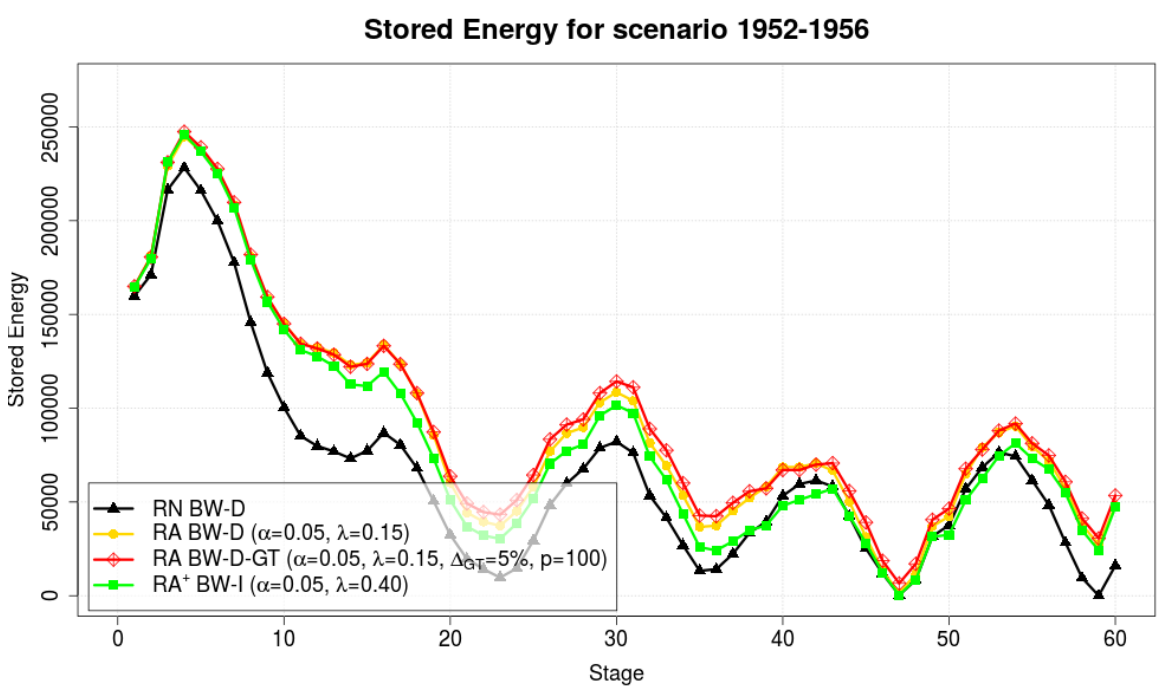

Figure 7.21: Total Brazilian stored energy for simulation with historical inflows occurred between 1952-1956

Total Deficit for scenario 1952-1956

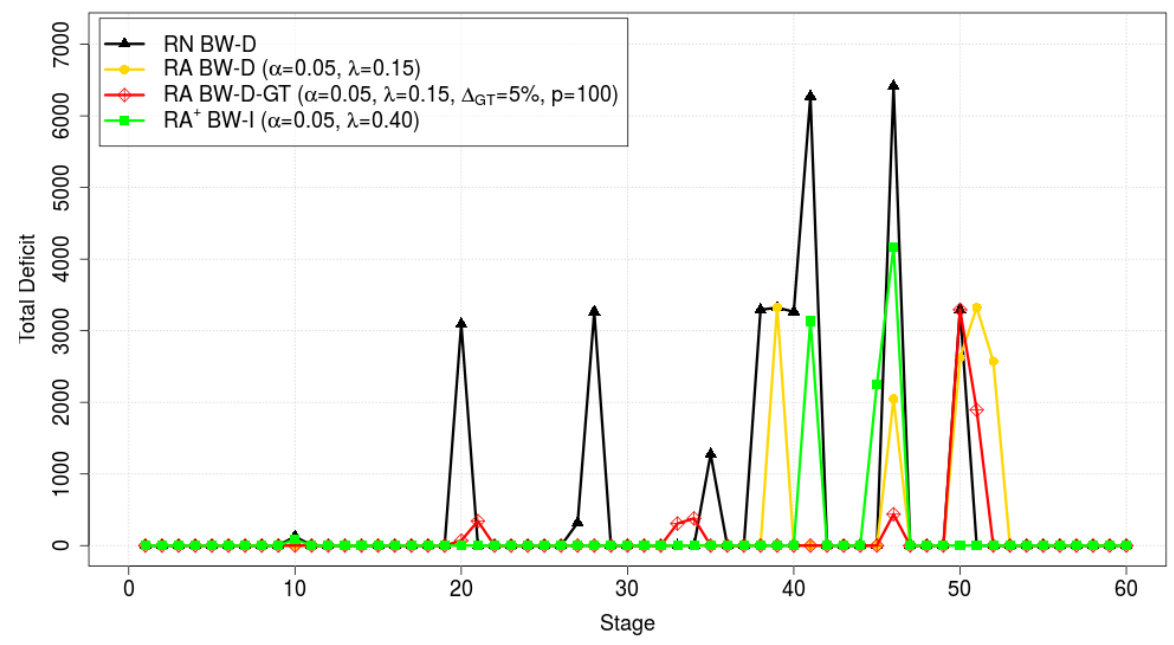

Figure 7.22: Total Brazilian energy deficit for simulation with historical inflows occurred between 1952-1956

to the better performance of "RA ${ }^{+}$BW-I" in both variability and cost based criteria. 


\section{Conclusions and Future Work}

\section{1}

\section{Conclusions}

In this work, it was shown that when an autoregressive model is used in the hydrothermal operation planning problem formulation, the variability of the solutions increases due to changes in the initial conditions of the time series model. Although the quality of the solutions are also increased by the consideration of such autoregressive models, the increase in the variability is often seen as an undesirable characteristic. In this context, the main focus of this work was to reduce the solution unpredictability and variability of Stochastic Dual Dynamic Programming (SDDP) method when applied to the hydrothermal operation planning problem.

Two approaches were proposed; the first one aimed at reducing primal solutions variability (thermal generations, in this case), and a second one with the objective of reducing both primal and dual solutions (in particular, thermal generation and PLDs - Portuguese translation of Differences Settlement Price, which are, in theory, the system's marginal costs).

The first proposal consists of considering the total thermal generation of each system as a state variable in the problem and penalize its variation over time. The second one consists of solving the algorithm with an independent model in the backward procedure, to ensure solution variability reduction, and a realistic time series model in the forward procedure, to ensure good trial decisions for the backward step. Additionally, in order to deal with the lack of ability of the independent time series model to generate persistent low inflow sequences, a more severe risk averse parametrization is used.

In order to compare the alternatives with respect to three criteria variability, cost and security - several solution quality indexes were proposed. Computational results applied to a hydrothermal operation problem have shown that a significant uncertainty and variability reduction is achieved with both proposals. The first proposal, "RA BW-D-GT", stabilizes thermal generation and results in reduction of its unpredictability and variability. The second proposal, "RA+ BW-I", stabilizes both thermal generations and PLDs, and has shown the best results for this criterion. 
Regarding the cost based criterion, both proposed approaches show results that are comparable to the "RA BW-D" reference case, with the proposed approach "RA+ BW-I" again showing better results. The last investigated criterion was the system security, and presented satisfactory results by both proposed approaches (risks smaller than 1\%), with no clear evidence that this criterion can be used to choose within possible alternatives to solve the problem. It is important to mention that regarding this criterion, although the "RA+ BW-I" results were satisfactory, the risk averse backward dependent approaches performed better, and further investigation may be required to assess the behaviour of the independent approach in more extreme situations.

As a conclusion of the performed experiments, this thesis shows that it is possible to obtain solutions with smaller uncertainty and variability, while showing characteristics that are close to the ones obtained by standard way to solve the problem. Considering all results shown in this work, the recommend approach is the "RA" ${ }^{+} \mathrm{BW}-\mathrm{I}$ ". However, due to the fact that the experiments were based on a single operation planning problem using one initial condition, more investigation is required in order to assure that the recommended approach is able to guarantee the security of the system in different conditions, in particular very extreme initial conditions.

\section{2}

\section{Future Work}

In Section 3 several sources of solution variability were mentioned, some of which are physical and cannot be solved by a model change, such as the regularization capacity reduction and the thermal plants characteristics, and others that are somehow related to the problem modelling choices, such as the consideration of river basins with different behaviour within the same equivalent reservoir system, the choice to use a deterministic forecast in the first month together with a coupling between different inflows forecasting models, and last but not least, the consideration of past inflows in the state space of the problem.

In this work the only source of variability that was studied was the last one, and the results were very promising. However, as the problem is complex and there are still other variability sources to be investigated, some future works are suggested.

A first topic that is likely to give good results is the consideration of uncertainty in the weekly stages of the first month, which may reduce the solution variability, although we believe that it is not going to be close to the levels achieved by the presented proposal. Such belief is supported by the fact 
that, even with a scenarios tree in the first month, the scenarios would still be biased by the past inflows information and would then change frequently over time. It is important to mention that, without empirical evidence, it is hard to be sure about the results that are going to be achieved by this approach. On the other hand, the potential improvement to the problem modelling brought by the consideration of the uncertainties in the first month is so big that this topic is an interesting future work, even if the solution variability is not reduced at all.

Another future investigation is regarding the impact of the coupling between a rain-flow model and a pure statistical one, such as an autoregressive model. This coupling, as mentioned before, is a likely cause of variability increase in the problem solution.

As a final suggestion, in order to better evaluate the impact of the approach proposed in this work, and also the ones suggested in this Section, a rolling horizon experiment comprising several years would give a better understanding of how much the solution variability would be decreased in practice, when one optimization problem is solved at the beginning of each week. 
A

\section{Publications}

During the development of this thesis several works considering the SDDP methodology were published. Some of these works, though not directly related to the main focus of this thesis, provide the basis on which the ideas of this thesis were developed.

\section{Complete papers published in journals}

- Shapiro, A., Tekaya, W., Paulo da Costa, J. and Pereira Soares, M., Risk neutral and risk averse Stochastic Dual Dynamic Programming method, European Journal of Operations Research, vol. 224, pp. 375-391, 2013.

- Shapiro, A., Tekaya, W., Pereira Soares, M. and Paulo da Costa, J., Worst-case-expectation approach to optimization under uncertainty, Operations Research, v. 61, p. 1435-1449, 2013.

\section{Complete paper to be submitted to journal}

- Soares, M. P., Street, A. and Valladão, D. M., On the solution variability reduction of SDDP applied to energy planning, to be submitted.

\section{Complete papers presented on Conferences}

- Soares, M. P., Street, A. and Valladão, D. M., On the solution variability reduction of SDDP applied to energy planning, IEEE PES Power General Meeting 2014, Washington, USA, presented by Murilo Soares.

- Soares, M. P., Street, A. and Valladão, D. M., Redução da Variabilidade da Solução da Programação Dinâmica Dual Estocástica Aplicada ao Planejamento da Operação de Sistemas Hidrotérmicos, IEEE INDUSCON 2014, Juiz de Fora, Brazil, presented by Murilo Soares. 


\section{Abstracts presented on Conferences}

- Shapiro, A., Tekaya, W., Pereira Soares, M. and Paulo da Costa, J., Worst-case-expectation approach to optimization under uncertainty, ICSP 2013, Bergamo, Italy, presented by Wajdi Tekaya.

- Shapiro, A., Tekaya, W., Paulo da Costa, J. and Pereira Soares, M. SDDP algorithm for Brazilian Power System Generation, SESO 2014 International Thematic Week, Paris, France, presented by Alexander Shapiro. 


\section{B}

\section{Autoregressive model impact on a general problem}

The effect of considering autoregressive models within SDDP was discussed on Chapter 4, where it was shown, by a simple example, that the relative importance of first stage inflows is increased if such models are considered. In this Appendix we are going to shown an intuitive way to estimate the impact of the inflow of stage $t$ in a general problem, with $T$ stages and autoregressive models with orders higher than one.

In order to reach a closed formula to estimate this impact, we are going to proceed by steps, assessing the impact $I_{t}$ of inflows of stage $t, a_{t}$, on each stage separately and, then, writing the cumulative effect. We are assuming that the inflows variables are already energy inflows with a coefficient equal to one in the RHS of hydro balance equations, such as in the examples used in this work. The impact for each stage $t+i$ can be calculated in a recursive way, as follows:

- Stage $t$ :

On stage $t$, the inflow $a_{t}$ has the same impact as the initial stored volume. In this case, let us assume a unitary impact $I_{t}=1$.

- Stage $t+1$ :

On stage $t+1$, we are interested on the impact of stage $t$ inflows. On the problems of this stage, $a_{t}$ appears as regressors in the autoregressive model at the RHS of hydro balance equation. Then, the impact is going to be $I_{t+1}=\phi_{t+1,1}$, where $\phi_{t+1,1}$ is the first autoregressive coefficient of the stage $t+1$ model.

- Stage $t+2$ :

On stage $t+2$, we are interested on the impact of stage $t$ inflows. On the problems of this stage, $a_{t+1}$ and $a_{t}$ appears as regressors. The impact of the second of the regressors is $\phi_{t+2,2} \cdot I_{t}$, and the first one is given by $\phi_{t+2,1} \cdot I_{t+1}$. Then, the impact is going to be $I_{t+2}=\phi_{t+2,1} \cdot I_{t+1}+\phi_{t+2,2} \cdot I_{t}$.

- Stage $t+i$ :

As a general rule, we can write the impact of inflows $a_{t}$ on stage $t+i$ as:

$$
I_{t+i}=\sum_{j=1}^{i} \phi_{t+i, j} \cdot I_{t+i-j}
$$


Anytime $j>p_{t+i}$, where $p_{t+i}$ is the stage $t+i$ model order, the coefficient $\phi_{t+i, j}$ is equal to 0 .

The impacts $I_{t+i}$ measure the relative impact of $a_{t}$ if compared to initial stored energy on stage $t+i, v_{t+i-1}$, referenced to stage $t+i$.

Our target now is to calculate the accumulated impact of $a_{t}$ in the problem, which can be done by the discounted sum of $I_{t+i}$ indexes from $t$ to $T$ considering that, at each stage, the impact $I_{t+i}$ is multiplied by the hydro balance equation average dual variable $\widetilde{\pi}_{h b, t+i}$. This variable is written without any additional indexes for simplification, but it can belong to different iterations and subproblems within SDDP solution process. The total impact is:

$$
I_{t}^{\text {total }}=\sum_{i=0}^{T-t} \tilde{\pi}_{h b, t+i} \cdot I_{t+i} \cdot \beta^{i} .
$$

This equation is not useful in the sense that there is no way to know $\widetilde{\pi}_{h b, t+i}$ values without solving the problem and getting to know the active cuts in the desired iteration, as it was done in the examples of Chapter 4.

In order to allow us to reach a useful expression, the hydro balance equation dual variable $\pi_{h b, t+i}$ is going to be dropped by, which is similar to consider that $\pi_{h b, t+i}=\pi_{h b, t+j}, \forall i, j$, and that the total impact calculated is a relative impact, that is, it is the impact of inflows over the impact of initial volume. This simplification is quite strong, but allows us to obtain a rough estimation of the relative impact.

The estimative is going to be:

$$
I_{t}^{t o t a l^{\prime}}=\sum_{i=0}^{T-t} I_{t+i} \cdot \beta^{i} .
$$

The impact index $I_{t}^{\text {total }}$ can be very useful in real applications, as it allows the estimation of the total relative impact of the inflows at each stage, and can be used to track whether the importance of inflows increases in some stages, for example. 


\section{C}

\section{Case study parametrization}

In the next two sections we are going to compare different parametrizations of the proposed approaches in order to choose one among them. In Section 7.2 we have compared the results of the best parametrizations of the two proposed approaches with risk neutral and risk averse case studies to show that we can reduce the solution variability of the problem and yet have good solutions when compared to the standard SDDP algorithm.

\section{C.1}

\section{Thermal generation stabilization}

The first approach to stabilize SDDP solutions was to augment the state space of the problem by adding variables that takes thermal generation into consideration. This allows us to add a penalty term in the objective function to limit variations in thermal generation over time.

In this experiment we have chosen a maximum thermal variability level of $5 \%$ over stages, and penalty term values $p \in\{50,100,250,500,1000\}$. The same parametrization was adopted in other experiment, this time penalizing only reductions of thermal generation. This approach allows the thermal generation to increase as fast as needed, but forces it to stay on for a longer period, as every reduction greater than $5 \%$ is penalized. The cases with a penalty term only for ramp-down variations are indicated by the variable $p_{d}$, and are shown below the cases in which a penalty is applied both directions.

In order to choose between the different solutions we are going to assess some of its properties. Our approach to choose between cases is going to be the following:

1. Policy value: this criterion is going to be used to discard expensive solutions but, once the solution has shown similar or cheaper results than standard risk averse case, it is not going to be used to rank the alternatives, except when we cannot rank them using the risks of deficit. This criterion considers only the operation costs and disregards any penalty costs that may appear in a certain problem.

2. Risks of deficit: this is going to be considered the most important criterion. The alternatives are going to be ranked regarding its comparison 
with standard risk averse case (which is our reference of security in this experiment).

3. Thermal generation and PLD volatilities: these criteria must be improved in comparison with standard approaches. In this comparison these criteria are going to be used to discard alternatives, but not to rank them.

This is not the only possible way to choose between the solutions, and not the most correct either. As this is in fact a multi-criteria problem, the relative importance of each criterion must be taken into account. In our case, we are considering that the most important objective is security, followed by an assumption that any proposed alternative should have an expected cost similar or smaller than our reference risk averse case. The third criterion, which is the main objective of this work, is the solution variability, which we require to be reduced by both proposed alternatives.

\section{C.1.1}

\section{Policy values}

The risk neutral approach has the lowest average cost, while the inclusion of risk averse consideration on the algorithm increased the cost by approximately 20\%, as shown in Figure C.1. This Figure also shows that limiting thermal generation variability with low penalty value $(p=50)$ results in a policy value that is already higher than the standard risk averse approach, and increases as the penalty term value increases. This result is expected, as the proposed formulation is equivalent to standard risk averse, with the addition of a penalty term that changes the objective function to consider another criterion. The result is that the operation cost minimization is less important in the new formulation and, then, a higher operation cost is expected to be found in the new solution. As the penalty cost increases, the importance of operation cost minimization decreases and the policy values are expected to increase. The candidate parametrizations are the ones with smaller penalty terms, $p=50$ and $p=100$ in the first experiment, and $p_{d}=50, p_{d}=100$ and $p_{d}=250$ in the second one. In an environment where the solution variability is worth increasing the expected policy value, higher values for the penalty could also be chosen.

\section{C.1.2}

\section{Risks of deficit}

Figure C.2 shows that all results are satisfactory in terms of security, with no way of distinguishing among the parametrizations. The solution variability 


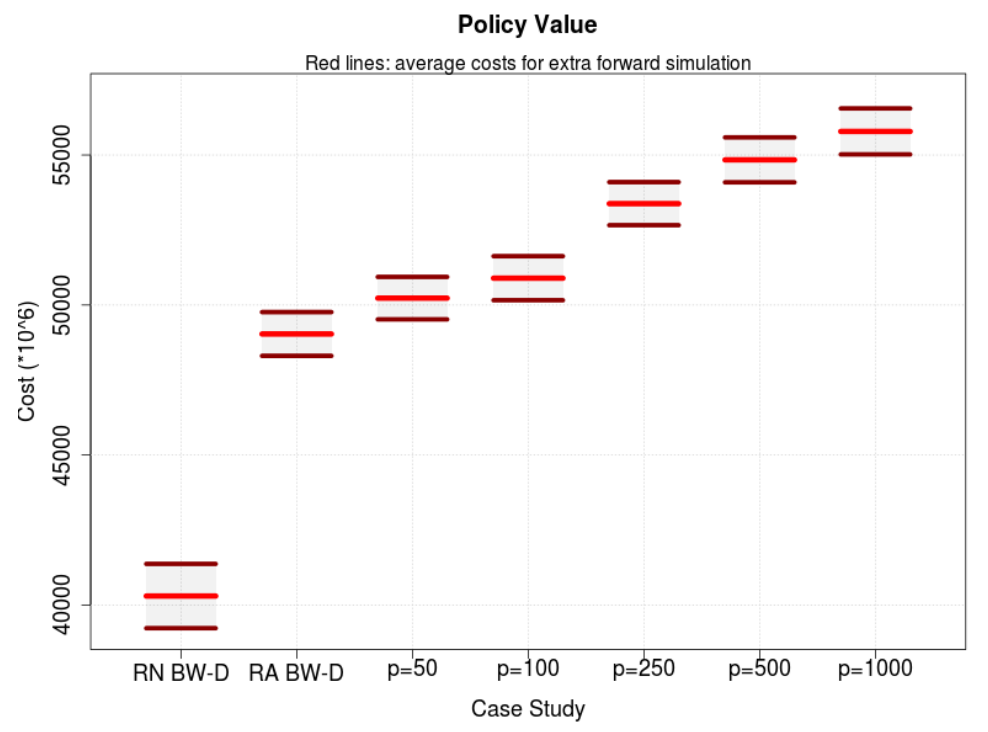

Policy Value

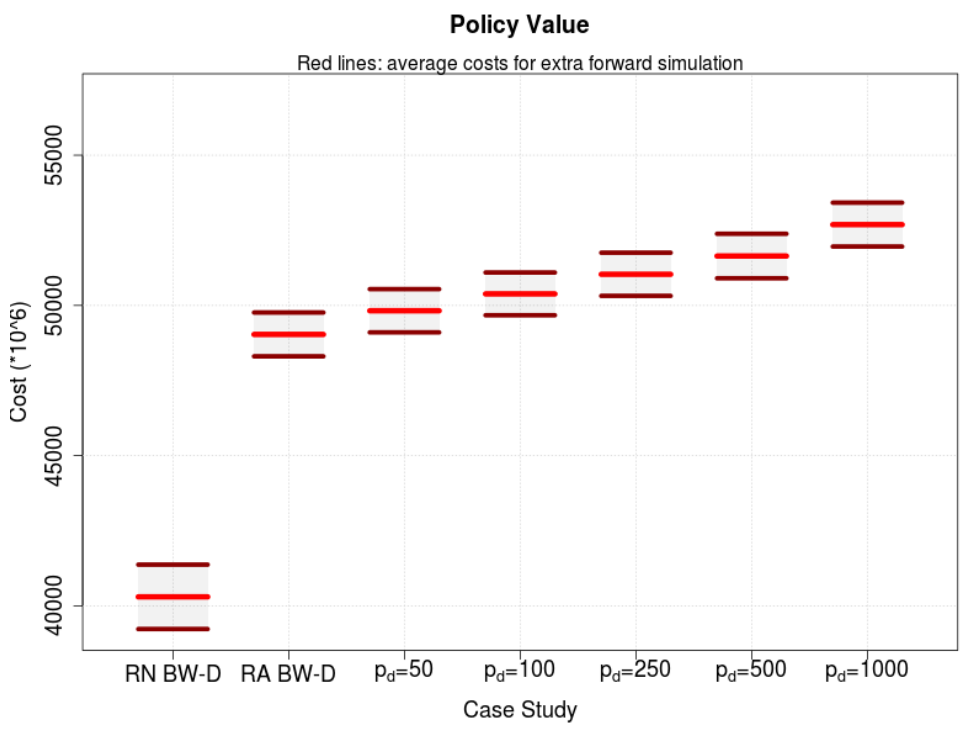

Figure C.1: Thermal generation approach - Policy values and 95\% confidence interval

indexes are going to be used to choose among them.

\section{C.1.3}

\section{Solution variability indexes}

\section{Thermal generation uncertainty index: 1-SAFE measure}

Thermal generation volatility over time can be measured by the procedure described in Section 6.1.1, Equation (6-1). The thermal generation volatility over time, as explicitly considered in the problem formulation, is very sensible to the penalty value. With a very high penalty value the solution variability is close to $5 \%$ with a small standard deviation. When the penalty value decreases, the average variability and its standard deviation increases, 


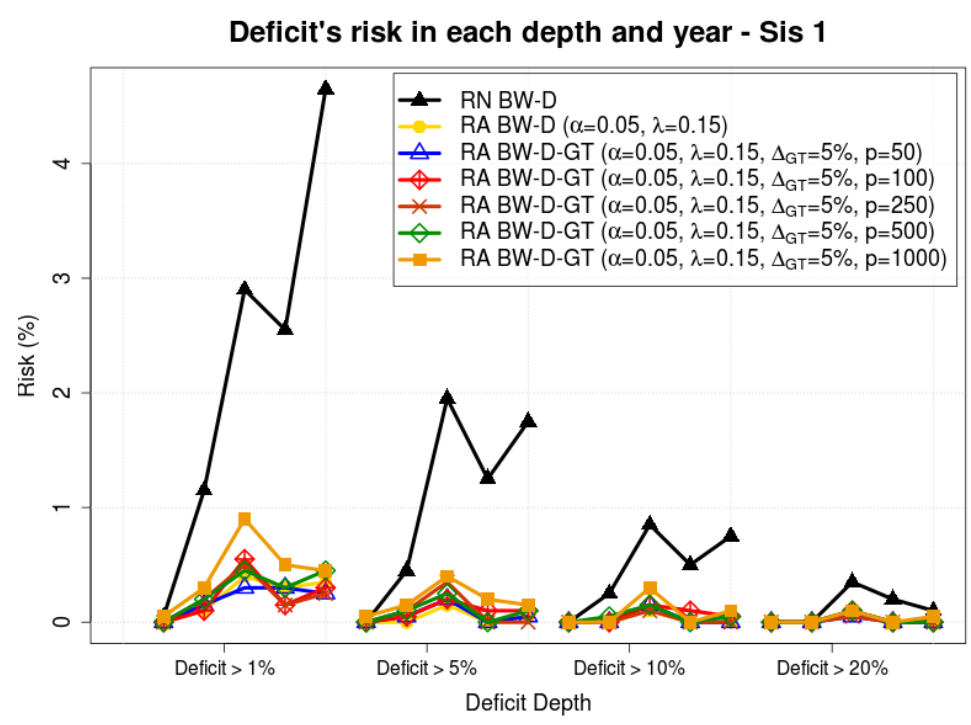

Deficit's risk in each depth and year - Sis 1

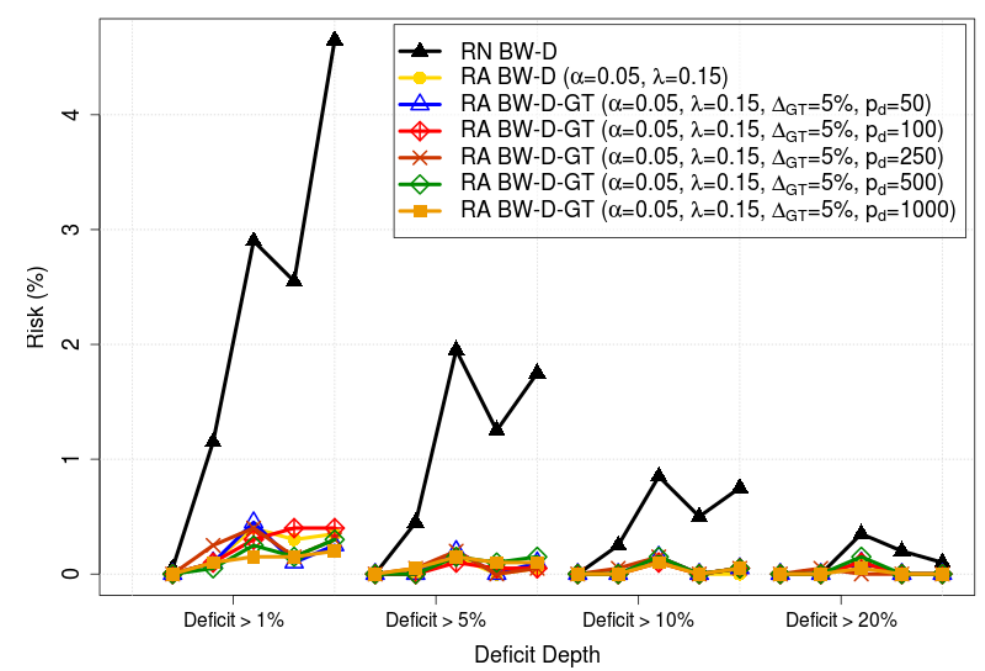

Figure C.2: Thermal generation approach - Annual risks of deficit

as expected, because in some scenarios the penalty term becomes too small to be able to keep variability within desired limits. Figure C.3 shows this result, where it is possible to see that the second candidate parametrization $(p=100)$ was able to reduce substantially the thermal generation variability without increasing the expected operation cost to prohibited levels. The $p=250$ solution, on the other hand, had good results regarding the solution variability, but has a bigger impact on the expected cost. The results of the second experiment, $p_{d}$, were not satisfactory, as the reduction achieved is too small if compared to the first experiment. This result may be explained by the fact that, although thermal generation reductions were controlled, the spikes due to the need to increase thermal generation in some cases contributed to keep the variability high. 

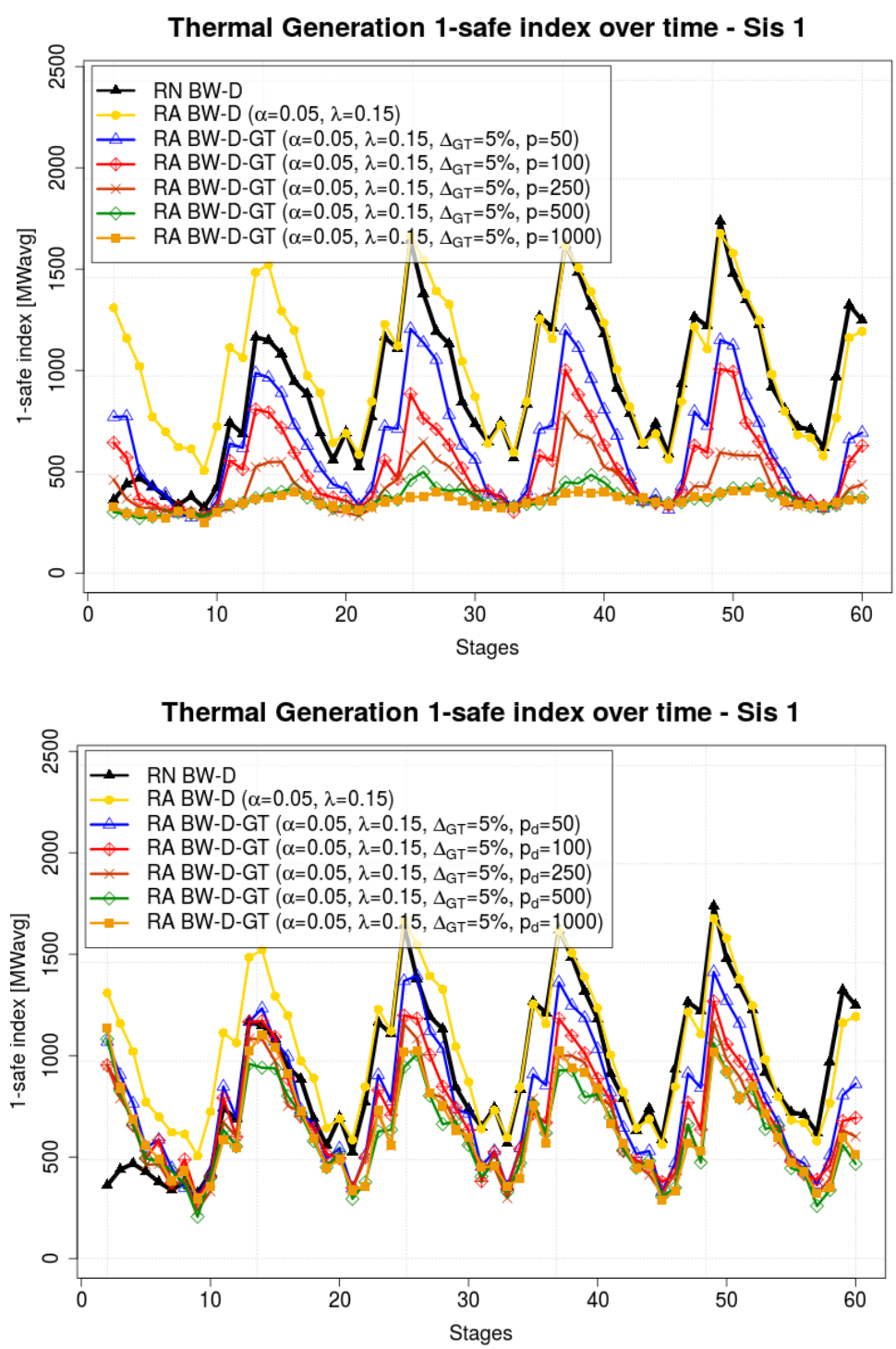

Figure C.3: Thermal generation approach - Thermal generation 1-SAFE index

\section{PLD uncertainty index: 1-SAFE measure}

The variability of PLD is not considered explicitly in this formulation and, as a consequence, its behaviour cannot be anticipated. Figure C.4 shows that there is no relation between the penalty term value and PLD uncertainty. 


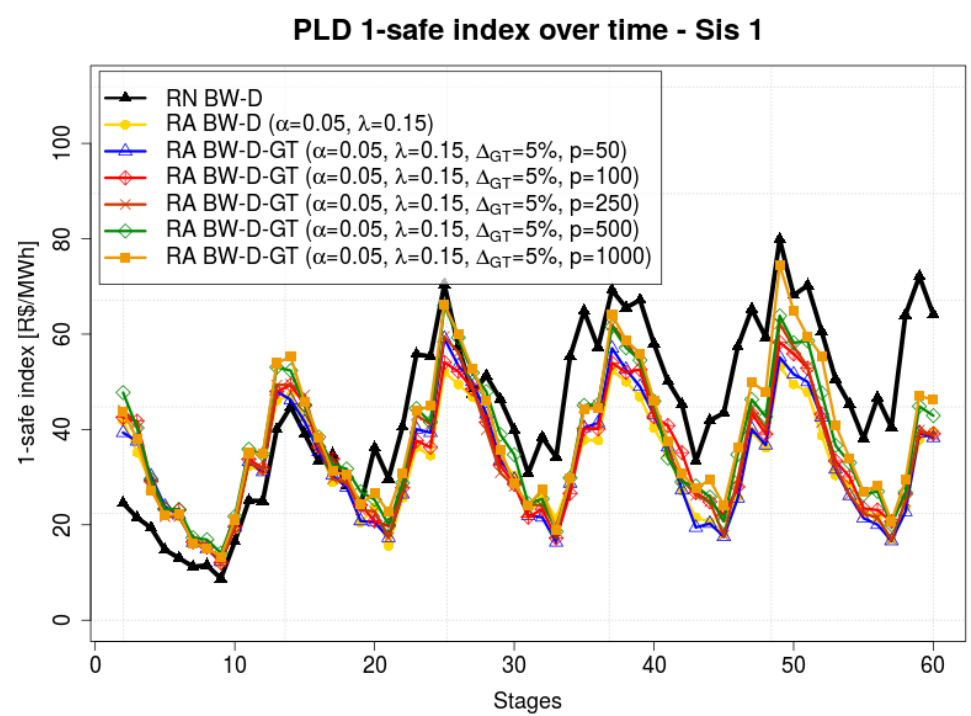

PLD 1-safe index over time - Sis 1

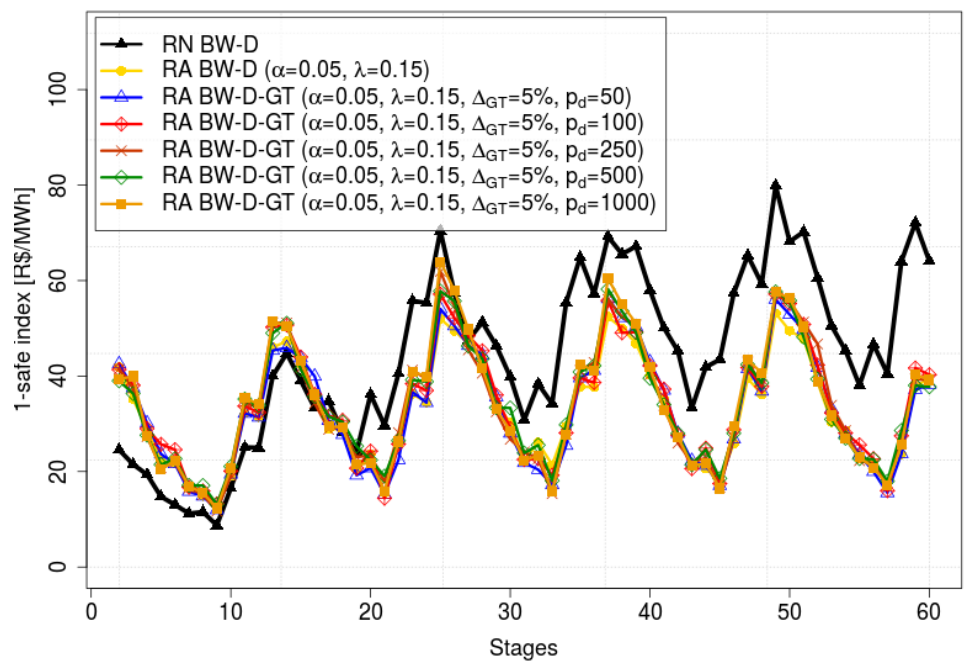

Figure C.4: Thermal generation approach - PLD 1-SAFE index

\section{C.1.4}

\section{Conclusions}

Given the previous results, the chosen parametrization was $p=100$. This solution presents a good compromise between the increase in the expected policy value and the reduction in thermal generation variability, while keeping the security index, deficit risk, at the same level as in the reference case.

\section{2}

\section{Risk averse backward independent SDDP}

In order to chose a parametrization for the backward independent approach, several experiments were run, considering different weights for risk aversion $(\lambda \in\{0.30,0.35,0.40,0.50\})$ and a fixed $\alpha=0.05$. The choice among the solutions was done using the same approach as before. 


\section{C.2.1}

\section{Policy values}

Figure C.5 shows the average policy values, together with its $95 \%$ confidence interval, for "RN BW-D", "RA BW-D ( $\alpha=0.05, \lambda=0.15)$ " and all four $\lambda$ parametrizations of the "RA BW-I" case. Regarding the backward independent approach, all solutions but $\lambda=0.50$ are satisfactory in the sense that they have expected costs between both standard approaches, while the $\lambda=0.50$ increases the expected cost to an unjustifiable level.

Policy Value

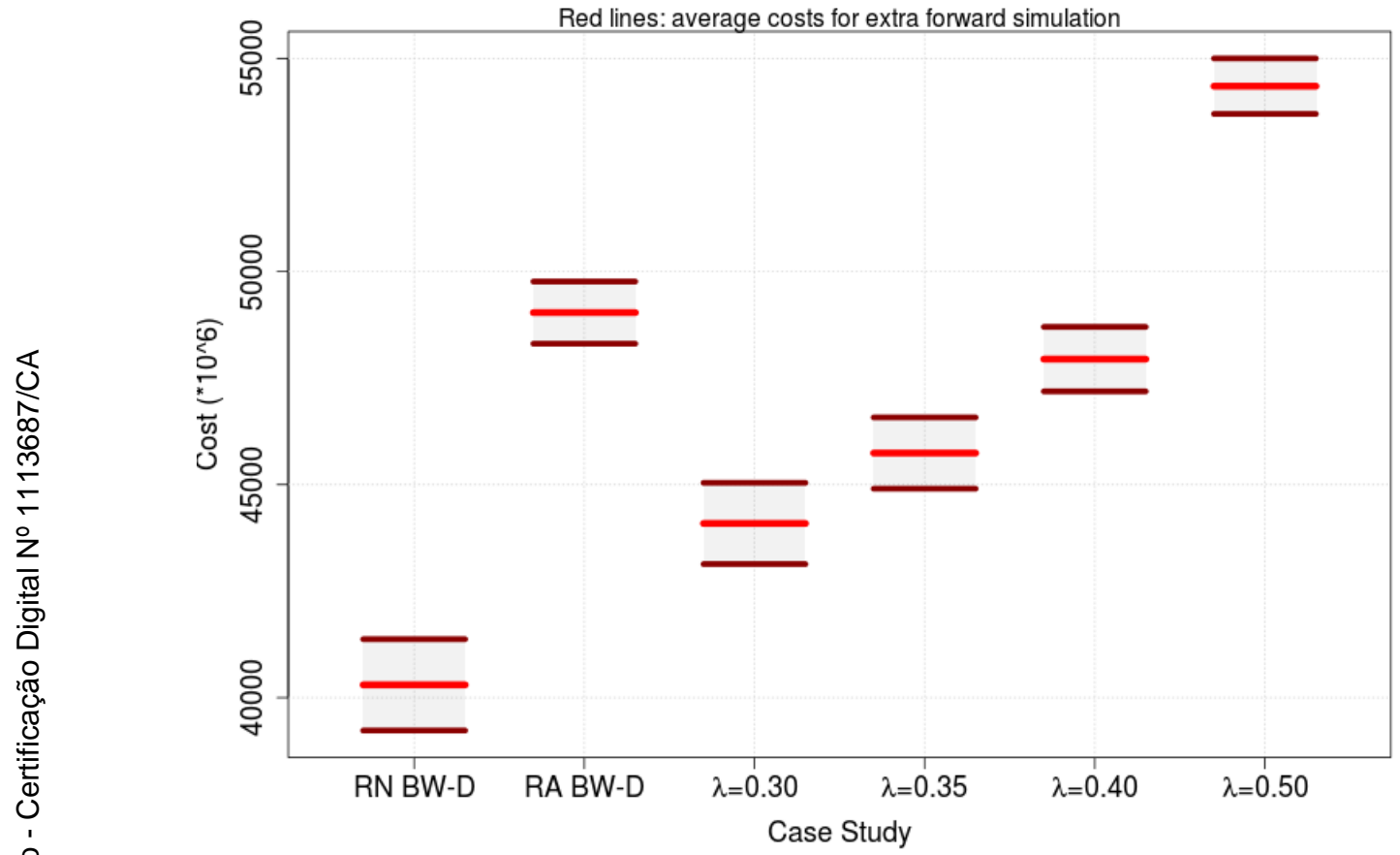

Figure C.5: BW-I approach - Policy values and 95\% confidence interval

\section{C.2.2}

\section{Risks of deficit}

Figure C.6 depicts the annual risks of deficit, for several deficit depths, that is, greater than $1 \%, 5 \%, 10 \%$ and $20 \%$ of the demand. The best result was shown by the backward independent case with $\lambda=0.50$, but, as already mentioned, the cost of this parametrization is too high. The better backward independent parametrization, in this case, is the one given by $\lambda=0.40$, for which all risks are bellow $1 \%$ and less than half of risk neutral value. 


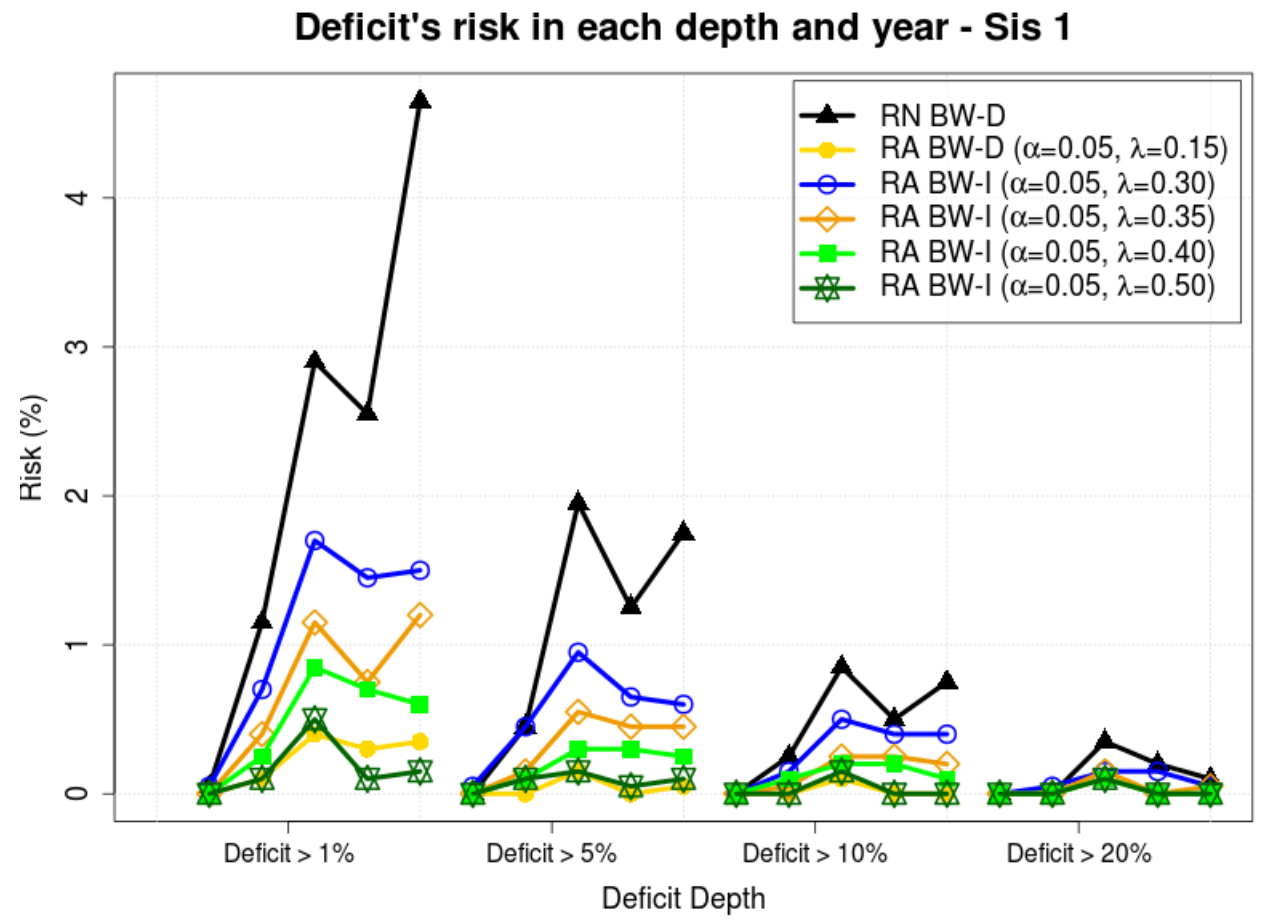

Figure C.6: BW-I approach - Annual risks of deficit

\section{C.2.3}

\section{Solution variability indexes}

The last variables that we are going to use to compare the parametrizations are thermal generation and PLDs.

\section{Thermal generation uncertainty index: 1-SAFE measure}

Figure C.7 shows the volatility results, and its clear that the best result is given by $\lambda=0.30$ approach, and it becomes worse as we increase $\lambda$ value. With the exception of $\lambda=0.50$ case, the others have shown a significant reduction in the variability compared to both standard cases.

\section{PLD uncertainty index: 1-SAFE measure}

Another important result is the volatility of PLDs, which, although derived from a dual variable, is also desirable to be stabilized. Figure C.8 shows that this objective is accomplished by all backward independent parametrizations. Indeed, the results are very similar between them, with only $\lambda=0.50$ case being slightly different. 


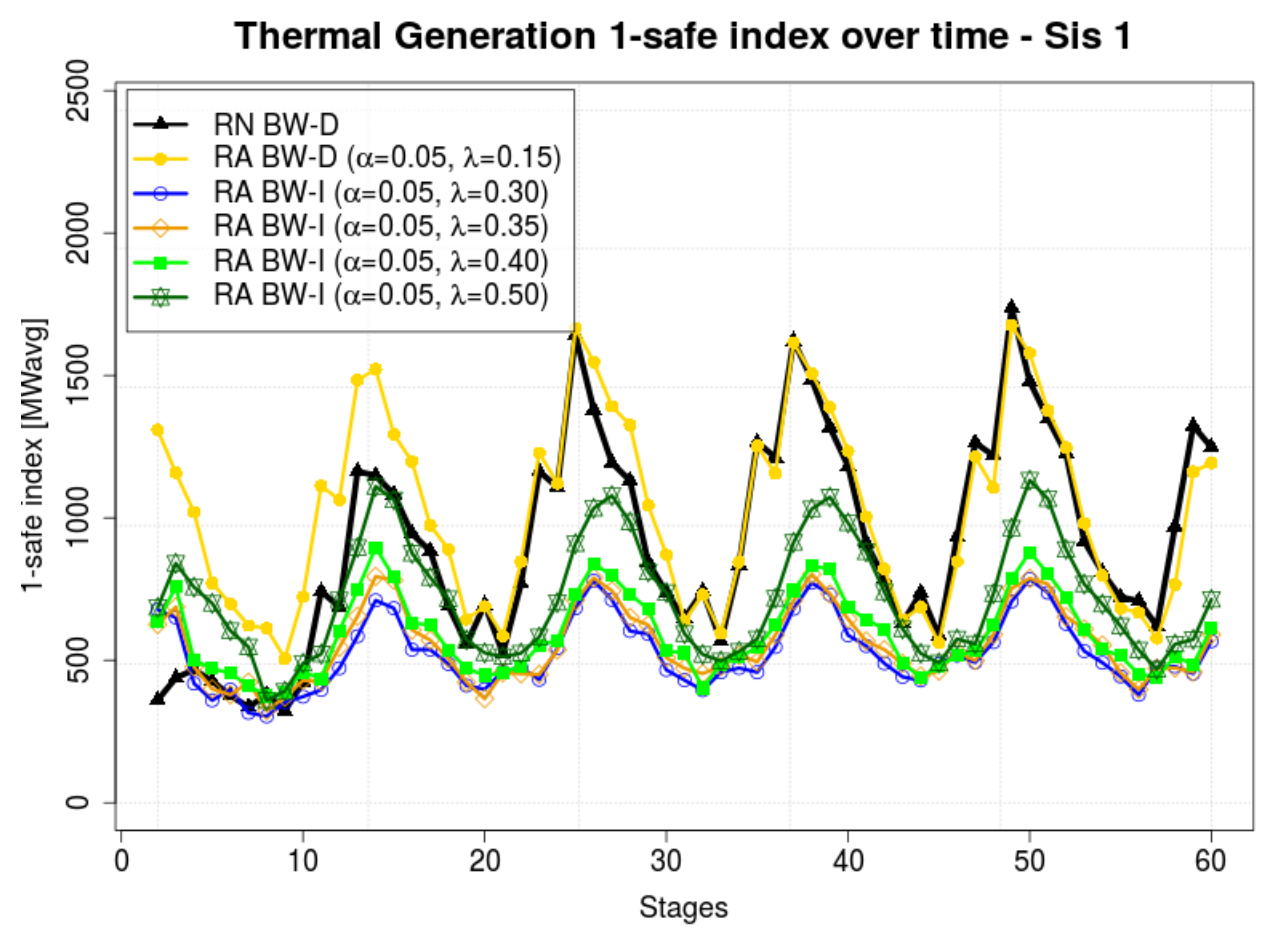

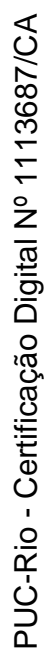

Figure C.7: BW-I approach - Thermal generation 1-SAFE index

PLD 1-safe index over time - Sis 1

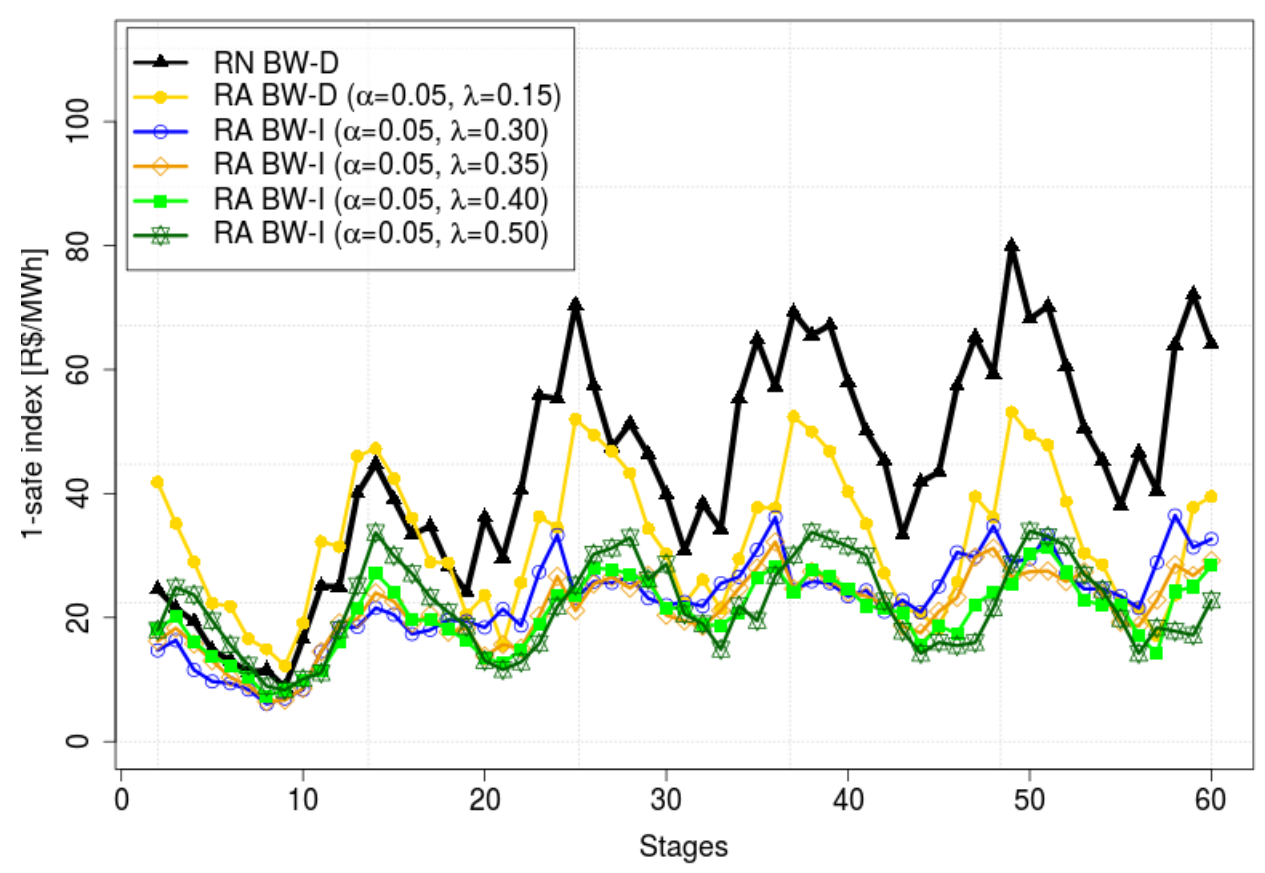

Figure C.8: BW-I approach - PLD 1-SAFE index

\section{2.4}

\section{Conclusions}

Given the presented results, the choice of one parametrization should be straightforward. Policy values results were responsible for taking out the 
$\lambda=0.50$ case due to its excessively high cost. The next result, risks of deficit, although couldn't discard any approach, has been better performed by case $\lambda=0.50$ (already discarded), followed by $\lambda=0.40$, and so on. The volatility indexes show great reduction in most stages for the proposed approach, and cannot be used to discard any parametrization in particular. The selected parametrization to be used in this work, due to its compromise between security, operation cost and solution variability reduction, is $\lambda=0.40$. 


\section{Bibliography}

[1] M. Maceira, L. Terry, F.S.Costa, J.M.Damázio, and A.C.G.Melo, "Chain of optimization models for setting the energy dispatch and spot price in the Brazilian system," Power System Computation Conference, no. June, pp. 24-28, 2002.

[2] M. Maceira, V. Duarte, D. Penna, L. Moraes, and A. Melo, "Ten years of application of stochastic dual dynamic programming in official and agent studies in Brazil - Description of the NEWAVE program," in 16th Power Systems Computation Conference - PSCC, 2008.

[3] M. Pereira and L. Pinto, "Multi-stage stochastic optimization applied to energy planning," Mathematical Programming, vol. 52, pp. 359-375, 1991.

[4] L. Barroso, J. Rosenblatt, A. Guimaraes, B. Bezerra, and M. Pereira, "Auctions of contracts and energy call options to ensure supply adequacy in the second stage of the Brazilian power sector reform," in 2006 IEEE Power Engineering Society General Meeting, p. 8 pp., IEEE, 2006.

[5] L. A. Barroso, A. Street, S. Granville, and M. V. Pereira, "Offering Strategies and Simulation of Multi-Item Iterative Auctions of Energy Contracts," IEEE Transactions on Power Systems, vol. 26, pp. 1917-1928, Nov. 2011.

[6] A. Shapiro, "Analysis of stochastic dual dynamic programming method," European Journal of Operational Research, vol. 209, pp. 63-72, Feb. 2011.

[7] ONS, "Operador nacional do sistema elétrico - dados relevantes 2012," December 2012 [accessed 06-November-2013].

[8] N. Arvanitidits and J. Rosing, "Composite representation of a multireservoir hydroelectric power system," Power Apparatus and Systems, IEEE, no. 2, pp. 319-326, 1970.

[9] N. Arvanitidis and J. Rosing, "Optimal operation of multireservoir systems using a composite representation," Power Apparatus and Systems, IEEE, no. 2, pp. 327-335, 1970. 
[10] C. M. V. D. B. Mércio, Resolução de problemas de planejamento de sistemas hidrotérmicos com representação do sistema por modelo equivalente de energia adotando acoplamento hidráulico. PhD thesis, UFRJ, 2000.

[11] A. L. M. Marcato, Representação Híbrida de Sistemas Equivalentes e Individualizados para o Planejamento da Operação de Médio Prazo de Sistemas de Potência de Grande Porte. PhD thesis, PUC-RJ, 2002.

[12] C. M. Suanno, Operação Estocástica de Sistemas Hidrotérmicos com Representação da Produtividade Variável. PhD thesis, UFRJ, 1995.

[13] V. L. de Matos, Um Modelo Para o Planejamento Anual da Operação Energética Considerando Técnicas avançadas de Otimização Estocástica. PhD thesis, UFSC, 2012.

[14] A. Shapiro, W. Tekaya, J. P. da Costa, and M. P. Soares, "Risk neutral and risk averse Stochastic Dual Dynamic Programming method," European Journal of Operational Research, vol. 224, pp. 375-391, Jan. 2013.

[15] M. A. I. Hipel, K. W., Time series modelling of water resources and environmental systems. Elsevier, 2004.

[16] M. S. Mondal and S. A. Wasimi, "Generating and forecasting monthly flows of the ganges river with par model," Journal of Hydrology, vol. 323, no. 1 ? 4 , pp. $41-56,2006$.

[17] R. Charbeneau, "Comparison of the two- and three-parameter log normal distributions used in streamflow synthesis," Water Resources Research, vol. 14, pp. 149-150, Feb. 1978.

[18] M. Maceira, "Relatório técnico cepel 237-1993 - programação dinâmica dual estocástica aplicada ao planejamento da operação energética de sistemas hidrotérmicos com representação do processo estocástico de afluências por modelos autorregressivos periódicos," tech. rep., 1993.

[19] B. C. Maceira, M.E.P., "Streamflow model for hydroelectric systems," in Proceedings of 5th International Conference on Probabilistic Methods Applied to Power Systems, PMAPS, 1997.

[20] D. J. M. Maceira, M.E.P., "The use of $\operatorname{par}(\mathrm{p})$ model in the stochastic dual dynamic programming optimization scheme used in the operation planning of the brazilian hydropower system operation planning studies of the brazilian generating system," in Proceedings of 8th International 
Conference on Probabilistic Methods Applied to Power Systems, PMAPS, 2004.

[21] D. D. J. Penna, Definição da Árvore de Cenários de Afluências para o Planejamento da Operação Energética de Médio Prazo. PhD thesis, 2009.

[22] B. B. C. J. Lopes, J.E.G., "Smap: a simplified hydrological model, applied modelling in catchment hydrology," Water Resources Publications, 1982.

[23] R. V. Slyke and R.-B. Wets, "L-shaped linear programs with applications to control and stochastic programming," SIAM Journal on Applied Mathematics, vol. 19, pp. 638-663, 1969.

[24] R. T. Rockafellar and S. Uryasev, "Optimization of conditional value-atrisk," Journal of risk, pp. 1-23, 2000.

[25] A. Philpott and V. de Matos, "Dynamic sampling algorithms for multistage stochastic programs with risk aversion," European Journal of Operational Research, vol. 218, pp. 470-483, Apr. 2012.

[26] A. Shapiro and W. Tekaya, "Report for technical cooperation between Georgia Institute of Technology and ONS - Risk Averse Approach," Tech. Rep. May, 2011.

[27] D. D. J. Penna, A. L. Diniz, and M. E. Maceira, "A Direct Approach to Represent CVaR Constraints in the Long Term Hydrothermal Planning Problem (in Portuguese)," in SEPOPE, 2012.

[28] D. D. J. Penna, A. L. Diniz, and M. E. Maceira, "A Direct CVaR Approach with a Detailed Representation of Critical Scenarios for the SDDP Method Applied to Hydrothermal Generation Planning," in INFORMS General Meeting, 2012.

[29] M. E. P. Maceira, L. G. B. Marzano, D. D. J. Penna, A. L. Diniz, and T. C. Justino, "Application of CVaR risk aversion approach in the expansion and operation planning and for setting the spot price in the Brazilian hydrothermal interconnected system," in 19th Power Sytems Computation, Wroclaw, Poland, 2014.

[30] Duke Energy International, "Formação de Preços nas Regras da CCEE: Periodicidade do PLD," tech. rep., 2006.

[31] K. Linowsky and A. Philpott, "On the Convergence of Sampling-Based Decomposition Algorithms for Multistage Stochastic Programs," Journal of Optimization Theory and Applications, vol. 125, pp. 349-366, May 2005. 
[32] A. B. Philpott and Z. Guan, "On the convergence of stochastic dual dynamic programming and related methods," Operations Research Letters, vol. 36, pp. 450-455, July 2008.

[33] P. Girardeau, V. Leclere, and A. Philpott, "On the convergence of decomposition methods for multi-stage stochastic convex programs," Mathematics of Operations Research, 2014.

[34] N. J. . T. R. R. Leite, A. L. S.; Castro, "Preço spot de eletricidade: teoria e evidências do caso brasileiro," in IV Encontro de Economia Catarinense, 2010.

[35] R. B. Heideier, F. A. A. Prado, M. A. Saidel, and M. Z. Ueocka, "Avaliação da volatilidade do preço de curto prazo no Brasil e sua relação com a geração térmica," in $8^{\circ}$ Congresso Latino Americano de Geração e Transmissão de Energia Elétrica - CLAGTEE 2009, 2009.

[36] J. P. da Costa, G. C. de Oliveira, and L. F. L. Legey, "Reduced scenario tree generation for mid-term hydrothermal operation planning," in 2006 International Conference on Probabilistic Methods Applied to Power Systems, p. 7 pp.

[37] ONS, "Relatório executivo do programa mensal de operação," 2012-2013 [accessed 06-November-2013].

[38] C. Donohue and J. Birge, "The abridged nested decomposition method for multistage stochastic linear programs with relatively complete recourse," Algorithmic Operations Research, vol. 1, pp. 20-30, 2006.

[39] Z. L. Chen and W. B. Powell, "Convergent Cutting-Plane and PartialSampling Algorithm for Multistage Stochastic Linear Programs with Recourse," Journal of Optimization Theory and Applications, vol. 102, no. 3, pp. 497-524, 1999.

[40] M. Hindsberger and A. Philpott, "ReSa: A Method for Solving Multistage Stochastic Linear Programs," in SPIX Stochastic Programming Symposium, 2001.

[41] G. Infanger and D. P. Morton, "Cut sharing for multistage stochastic linear programs with interstage dependency," Mathematical Programming, vol. 75, pp. 241-256, Nov. 1996.

[42] M. V. F. Pereira and L. M. V. G. Pinto, "Stochastic Optimization of a Multireservoir Hydroelectric System: A Decomposition Approach," Water Resources Research, vol. 21, no. 6, pp. 779-792, 1985. 
[43] R. Kelman, Planejamento Coordenado dos Setores de Energia Elétrica e Gás Natural. PhD thesis, 2009.

[44] A. L. Diniz and M. E. P. Maceira, Stochastic Programming - Applications in Finance, Energy, Planning and Logistics: ed. by Horand I. Gassmann and William T. Ziemba, ch. Multi-lag Benders decomposition for power generation planning with nonanticipativity constraints on the dispatch of LNG thermal plants. World Scientific, 2013.

[45] C. de Pesquisas de Energia Elétrica Cepel, "Nota técnica cepel - set. 2002 - análise das variações nas gerações das usinas hidroelétricas - modelo dessem," tech. rep., 2002.

[46] C. de Pesquisas de Energia Elétrica Cepel, "Nota técnica cepel - fev. 2014 - modelagem das restrições internas de variação (rivar) no modelo dessem-pat," tech. rep., 2014.

[47] C. Donohue, Stochastic network programming and the dynamic vehicle allocation problem. $\mathrm{PhD}$ thesis, 1996.

[48] J. R. Birge, "Decomposition and partitioning methods for multistage stochastic linear programs," Operations Research, vol. 33, no. 5, pp. pp. 989-1007, 1985.

[49] K. Barty, "A note on the convergence of the SDDP algorithm," optimization-online.org, 2012.

[50] M. Hindsberger and A. B. Philpott, "Stopping criteria in sampling strategies for multistage slp-problems," Applied Mathematical Programming and Modelling, vol. 102, no. 3, pp. 497-524, 2002.

[51] A. Shapiro, W. Tekaya, J. P. Costa, and M. P. Soares, "Report for technical cooperation between Georgia Institute of Technology and ONS," Tech. Rep. April, 2011.

[52] T. Homem-de Mello, V. de Matos, and E. Finardi, "Sampling strategies and stopping criteria for stochastic dual dynamic programming: a case study in long-term hydrothermal scheduling," Energy Systems, 2011.

[53] R. Wunderling, "Paralleler und objektorientierter simplex," Tech. Rep. TR-96-09, ZIB, Takustr.7, 14195 Berlin, 1996.

[54] A. Philpott, V. de Matos, and Z. Guan, "Advances in DOASA," in 2010 EPOC Winter Workshop, 2010. 